Florida International University FIU Digital Commons

\title{
A robotic ankle-foot orthosis with integrated electromyography biofeedback control for improving gait symmetry in stroke rehabilitation
}

Szu-Min Chang

Florida International University

DOI: $10.25148 /$ etd.FI14060140

Follow this and additional works at: https://digitalcommons.fiu.edu/etd

Part of the Biomedical Engineering and Bioengineering Commons

\section{Recommended Citation}

Chang, Szu-Min, "A robotic ankle-foot orthosis with integrated electromyography biofeedback control for improving gait symmetry in stroke rehabilitation" (2007). FIU Electronic Theses and Dissertations. 2108.

https://digitalcommons.fiu.edu/etd/2108 
Miami, Florida

A ROBOTIC ANKLE-FOOT ORTHOSIS WITH INTEGRATED

ELECTROMYOGRAPHY BIOFEEDBACK CONTROL FOR IMPROVING GAIT SYMMETRY IN STROKE REHABILITATION

A thesis submitted in partial fulfillment of the

requirements for the degree of

MASTER OF SCIENCE

in

BIOMEDICAL ENGINEERING

by

Szu-Min Chang 
To: Dean Vish Prasad

College of Engineering and Computing

This thesis, written by Szu-Min Chang, and entitled A Robotic Ankle-Foot Orthosis with Integrated Electromyography Biofeedback Control for Improving Gait Symmetry in Stroke Rehabilitation, having been approved in respect to style and intellectual content, is referred to you for judgment.

We have read this thesis and recommend that it be approved.

Richard Schoephoerster

Eric Crumpler

Diana Rincon, Major Professor

Date of Defense: March 28, 2007

The thesis of Szu-Min Chang is approved.

Dean Vish Prasad
College of Engineering and Computing

Florida International University, 2007 
(C) Copyright 2007 by Szu-Min Chang

All rights reserved. 


\section{DEDICATION}

I dedicate this thesis to my family. To my mom and dad for sacrificing so much to provide me with everything I needed and more. To my brother who I always wanted to be like in one way or another. To my grandmother and grandfather who helped raise me and always wished the very best for me. And to my loving wife who has become my partner in life and my best friend. Thank you for always believing in me, supporting me through troubled times, and giving me the strength and direction to help me be a better person. I love you all and owe you everything I have in life. I hope I make you proud. 


\section{ACKNOWLEDGMENTS}

I would like to start by saying thank you to Dr. Diana Rincon for being my advisor through my career here at FIU. You've provided me with the necessary guidance and support in helping me complete my studies. I know it took a bit longer than we wanted, but I couldn't be any happier with what I've been able to accomplish.

To Dr. Leonard Elbaum for taking the time to help me develop my idea from the very beginning all the way through to the very end. You've shown me what a dedicated and inspirational teacher you are.

To Mr. Richard Zicarelli for showing me the tricks of the trade in the machine shop and for helping me create some of the more precise parts necessary for my device to come alive. Without them, the entire thing would only be just a very cool looking paper weight.

To Mr. Curtis Tom whom without I would probably have taken another 2 years to complete my thesis. You've provided me with the guidance I needed to get through the toughest and most complicated portion of my studies. I admire your experience and appreciate everything you have taught me.

To Dr. Richard Schoephoerster and Dr. Eric Crumpler for serving on my committee. Your opinions and sincere compliments mean a lot to me. Thank you for making my time at FIU a great learning experience.

And to all my fellow FIU Classmates, thank you for sharing your time and experiences with me through our studies together. It's an experience I won't soon forget. I wish everyone the best of luck towards their pursuit of happiness. 


\title{
ABSTRACT OF THE THESIS
}

\section{A ROBOTIC ANKLE-FOOT ORTHOSIS WITH INTEGRATED}

\section{ELECTROMYOGRAPHY BIOFEEDBACK CONTROL FOR IMPROVING GAIT \\ SYMMETRY IN STROKE REHABILITATION}

\author{
by \\ Szu-Min Chang \\ Florida International University, 2007 \\ Miami, Florida \\ Professor Diana Rincon, Major Professor
}

The pathology of stroke attacks the central nervous system commonly resulting in the loss of voluntary neuromuscular control which negatively affects the walking capability of many survivors. New rehabilitation techniques utilizing biofeedback, robotic assisted movements, and gait specific exercises have been shown individually to increase the neurological recovery as compared to traditional methods of physical rehabilitation. The Biofeedback Assisted Robotic Ankle-foot orthosis (B.A.R.A.) is a prototype that combines all three characteristic treatments into a single portable device focused on improving voluntary dorsiflexion capability and gait patterns of the ankle-foot complex. The initial pilot study utilized a single healthy individual to study the joint angle patterns of the ankle while utilizing the BARA device in a drop-foot gait simulation. The resulting angular positional curves showed general patterns representative of a normal gait cycle while eliminating the hazardous effects of drop-foot. 


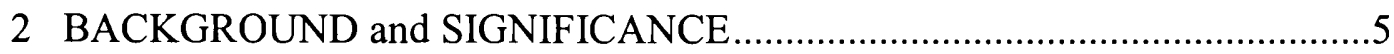

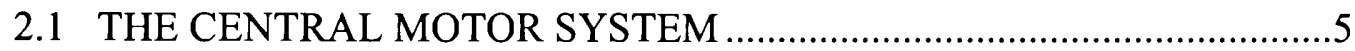

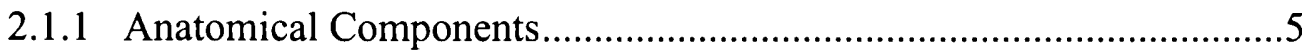

2.1.2 Sensory Pathways and Motor Pathways ...........................................

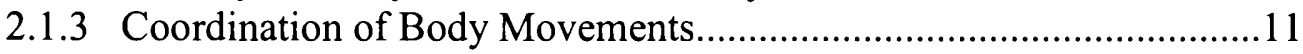

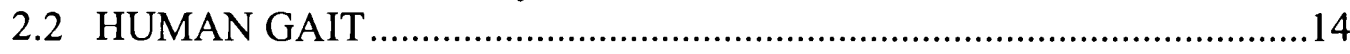

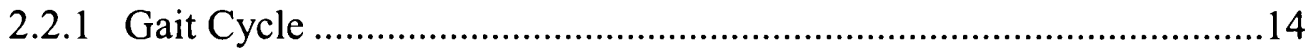

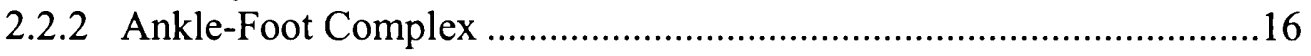

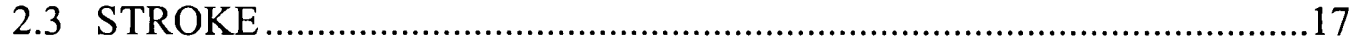

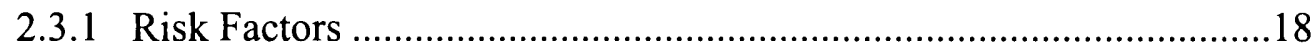

2.3.2 Neuromuscular Effects of Stroke..................................................19

2.3.3 Cortical Reorganization and Motor Learning ..................................20

2.4 GAIT REHABILITATION TREATMENTS FOR STROKE ..................20

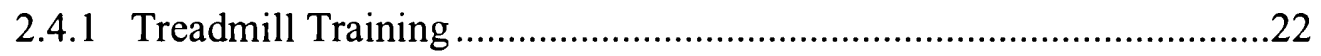

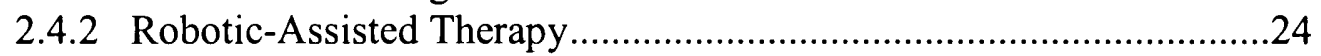

2.4.3 Electromyography Biofeedback ................................................25

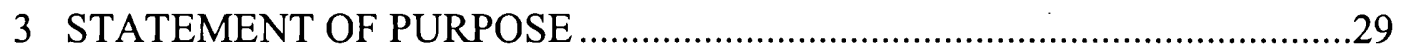

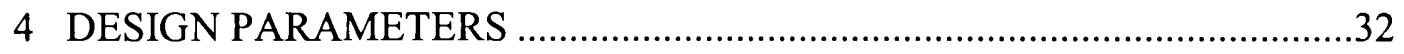

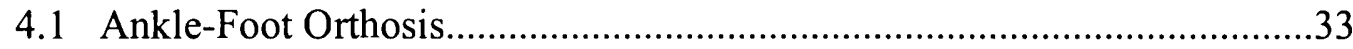

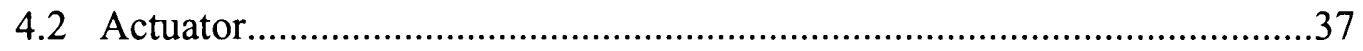

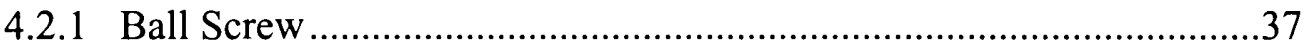

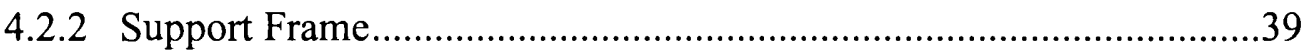

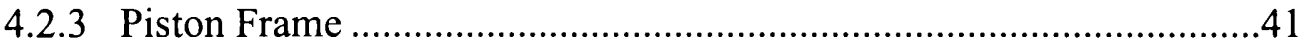

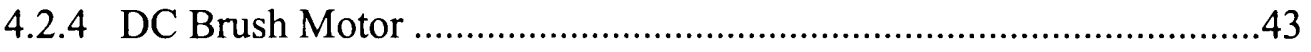

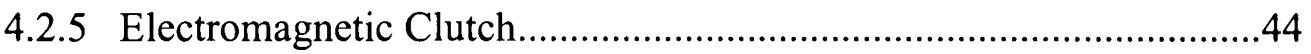

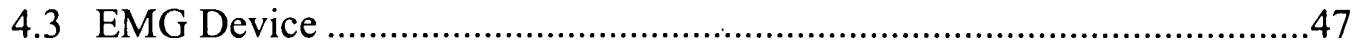

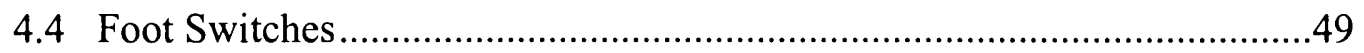

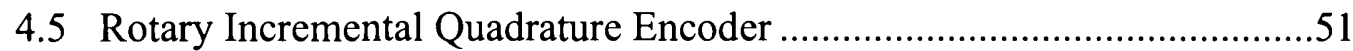

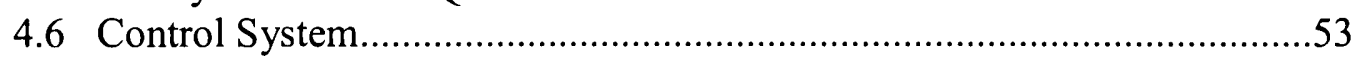

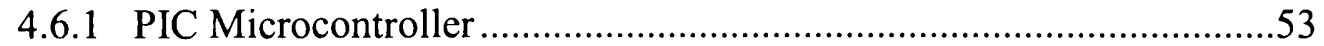

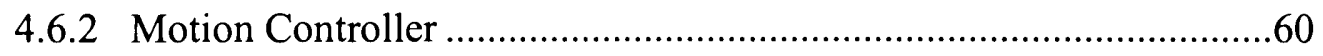

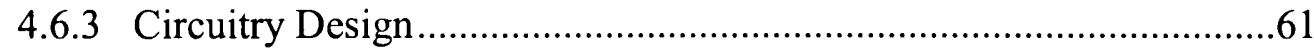

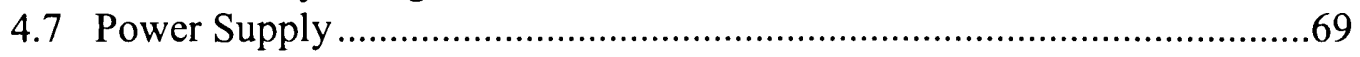

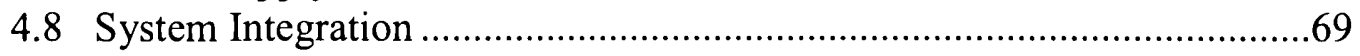

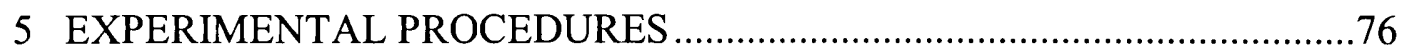

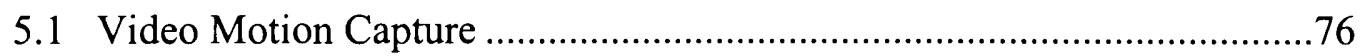


5.1.1 Calibration Frame .77

5.1.2 Marker Placement

5.2 Experimental Setup .79

5.2.1 Equipment Setup. .79

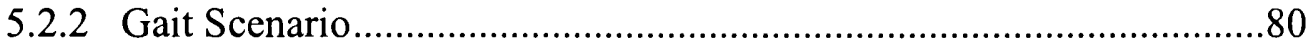

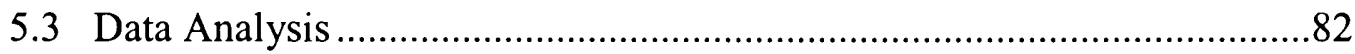

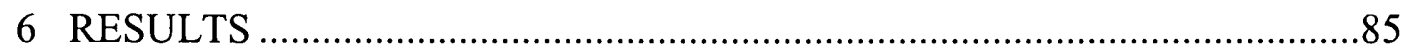

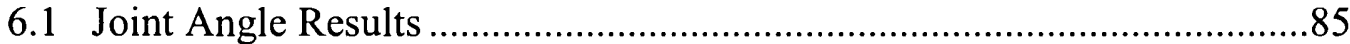

6.2 Stride Length and Gait Velocity Results .............................................92

7 DISCUSSION

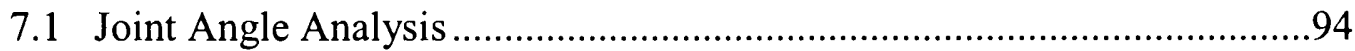

7.2 Stride Length and Gait Velocity Analysis ............................................98

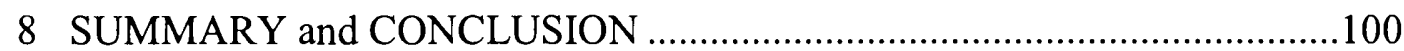

9 RECOMMENDATION AND FUTUREWORK …..........................................104

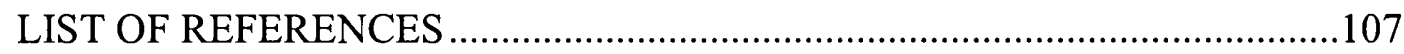

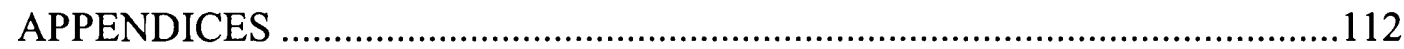


Table 2.1 Summary Description of the Phases within a Gait Cycle 16

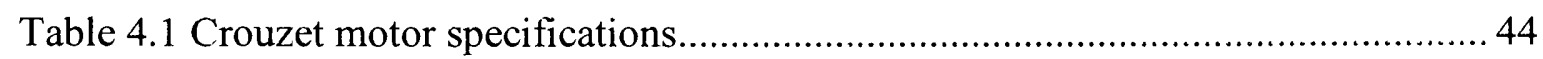

Table 4.2 SO-11 electromagnetic clutch cpecifications...............................................45

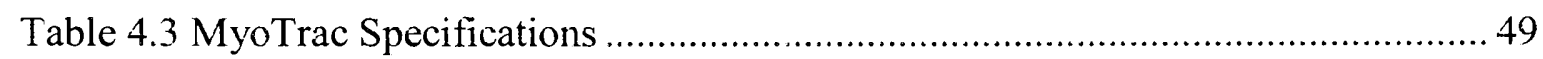

Table 4.4 Flexiforce load sensor model 1207 specification........................................ 50

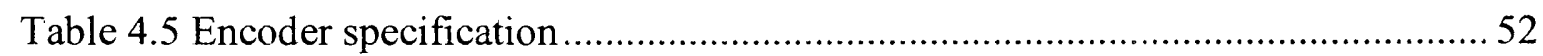

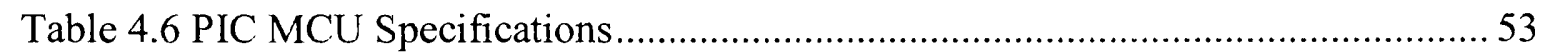

Table 4.7 PIC--Servo SC and Z232-485 Serial Port Converter Specifications................. 61

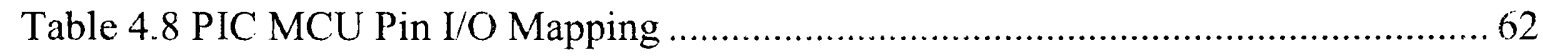

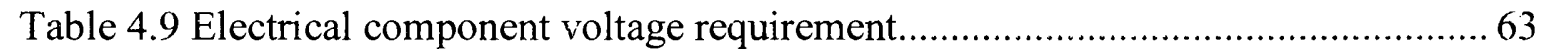

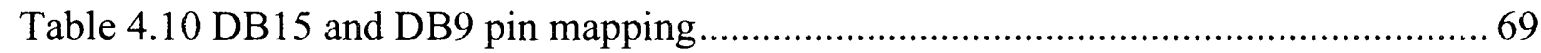

Table 4.11 Control Circuit Board components ................................................................ 72

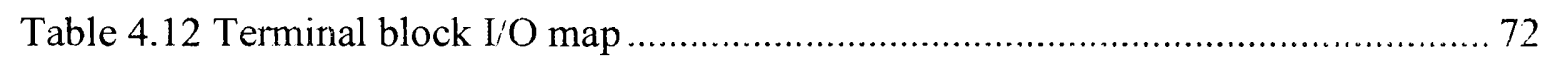

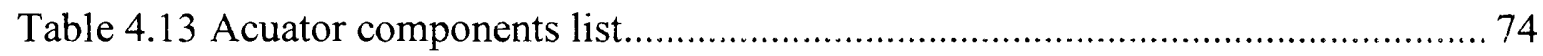

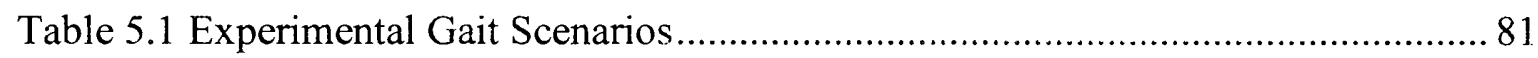

Table 6.1 Average values for duration of gait cycle, stride length, and walking

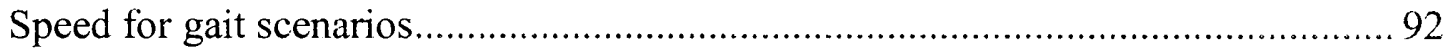




\section{LIST OF FIGURES}

FIGURE

Figure 2.1 Lateral view of the brain with selected anatomical components within the CNS responsible for motion (Martini et al. 2003).

Figure 2.2 Anterior view of the tibialis anterior muscles of the right leg (Martini, 2003).

Figure 2.3 Corticospinal Pathway (Martini et al. 2003). 10

Figure 2.4 Control sequence for voluntary movements within the CNS (Martini et al. 2003).

Figure 2.5 Stick figure illustration of the individual phases within a gait cycle for

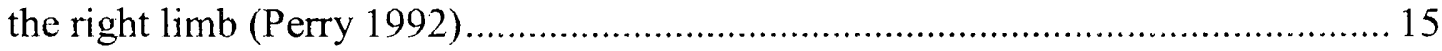

Figure 2.6 Mean Ankle Range of Motion during Gait Cycle (Perry 1992) ...................... 17

Figure 2.7 Body Weight Support Treadmill Training..................................................... 23

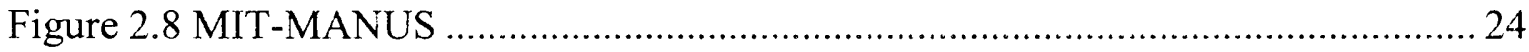

Figure 2.9 Electromyography Biofeedback Training............................................. 26

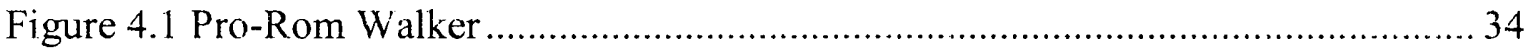

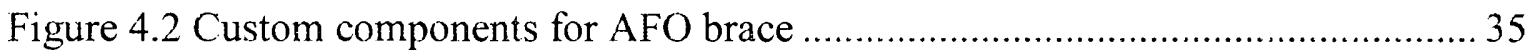

Figure 4.3 Assembled Ankle-Foot Orthosis with custom components attached .............. 36

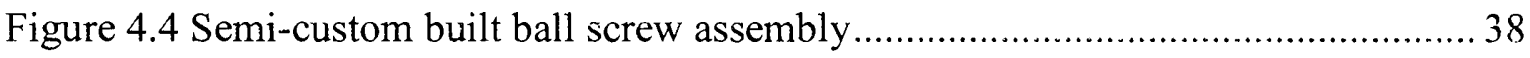

Figure 4.5 Assembled actuator support frame …..................................................... 40

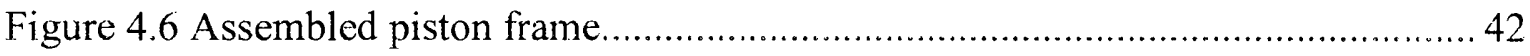

Figure 4. 7 Crouzet DC brushed motor dimension ................................................ 43

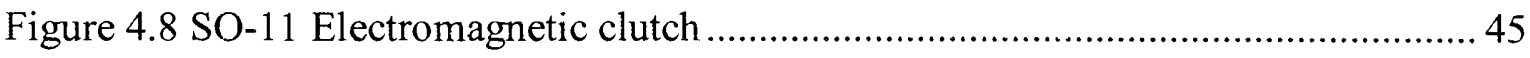

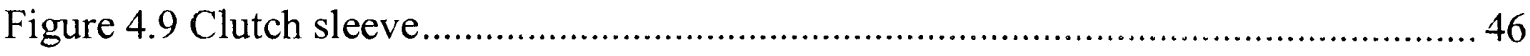

Figure 4.10 Exploded view of clutch assembly components ......................................47 
Figure 4.11 Myotrac EMG device with sensor cable and Triode 48

Figure 4.12 Foot switch components and assembly ................................................. 51

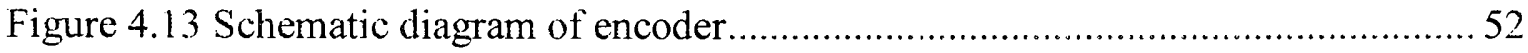

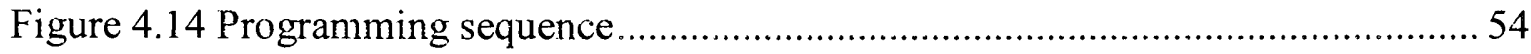

Figure 4.15 Logic flowchart for BARA (see Appendix B for full size image).................56

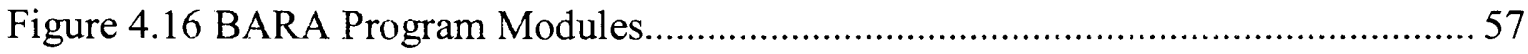

Figure 4.17 PIC-Servo SC and z232-485 serial port converter................................... 61

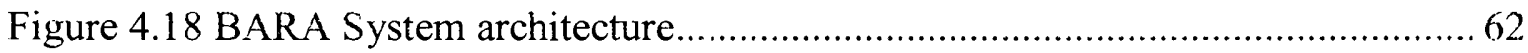

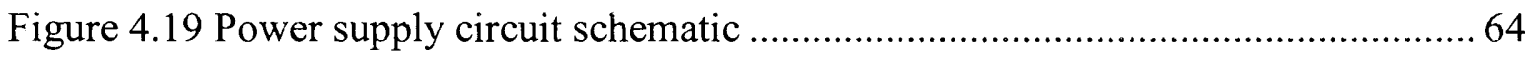

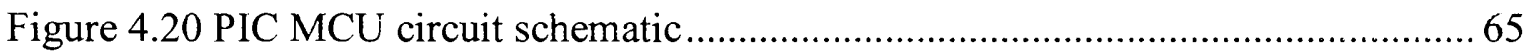

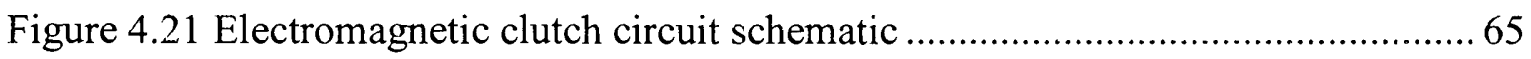

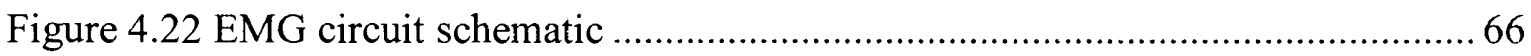

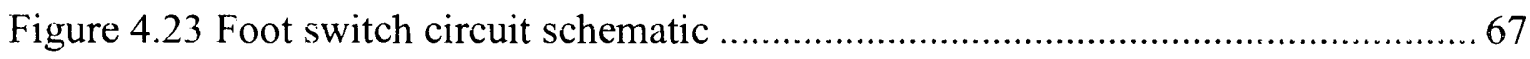

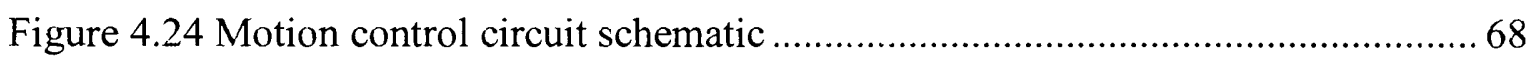

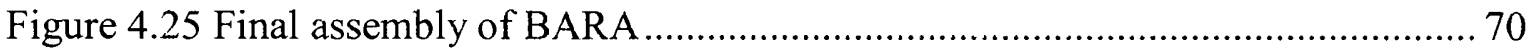

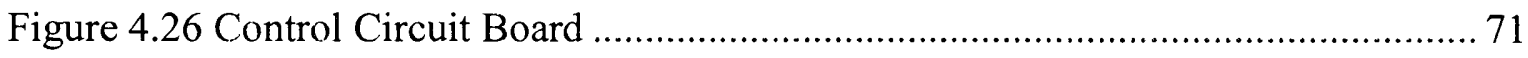

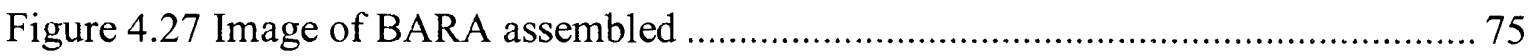

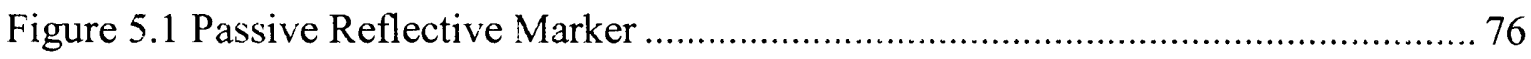

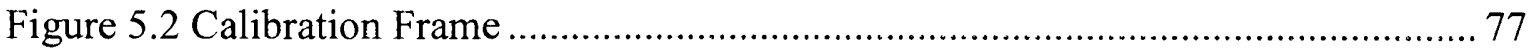

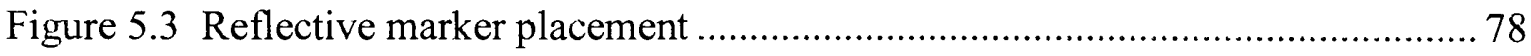

Figure 5.4 Video motion capture equipment layout.................................................... 79

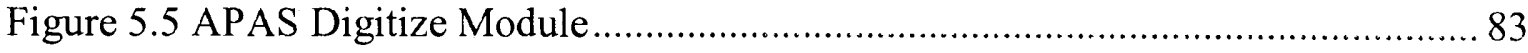


Figure 6.1 Joint angle of right leg for normal barefoot walking (full data set).......

Figure 6.2 Joint angle of right leg for simulated drop-foot barefoot walking (full data set) 86

Figure 6.3 Joint angle of right leg for normal walking with BARA powered-off (full data set).

Figure 6.4 Joint angle of right leg for simulated drop-foot walking with BARA powered-off (full data set).

Figure 6.5 Joint angle of right leg for simulated drop-foot walking while using BARA assisted movements (full data set).

Figure 6.6 Joint angle of right leg for normal barefoot walking (single gait cycle) 88

Figure 6.7 Joint angle of right leg for simulated drop-foot barefoot walking (single gait cycle)

Figure 6.8 Joint angle of right leg for normal walking with BARA powered-off (single gait cycle) 89

Figure 6.9 Joint angle of right leg for simulated drop-foot walking with BARA powered-off (single gait cycle)

Figure 6.10 Joint angle of right leg for simulated drop-foot walking while using BARA activated (single gait cycle).

Figure 6.11 Cumulative joint angle curves results of Trial 1 for all the gait scenarios

Figure 6.12 Average time to complete a single gait cycle

Figure 6.13 Average stride length of gait cycle 93

Figure 6.14 Average gait velocity 93

Figure7.1 Effects of Parallax on digitization of BARA video images .96 
ABBREVIATION TERMS

$\mathrm{AC}$

ADL

AFO

BARA

BFB

BWS

CNS

CPU

CVA

CVD

DC

DOF

EMG

GC

HBP

$\mathrm{I} / \mathrm{O}$

MCU

NDT

ROM

SNS

PAD

PNS
Alternating Current

Activities of Daily Living

Ankle Foot Orthosis

Biofeedback Assistive Robotic Ankle-foot orthosis

Biofeedback

Body Weight Support

Central Nervous System

Central Processing Unit

Cerebral Vascular Accident

Cardiovascular Disease

Direct Current

Degree-of-Freedom

Electromyography

Gait Cycle

High Blood Pressure

Input/Output

Microcontroller

Neurodevelopment Technique

Range Of Motion

Somatic Nervous System

Peripheral Arterial Disease

Peripheral Nervous System 


\section{INTRODUCTION}

Cardiovascular Disease (CVD) is a class of disease that can affect both the heart and blood vessels of an individual at risk. The condition is linked to a variety of common health factors such as high blood pressure (HBP), high cholesterol, obesity, physical inactivity, diabetes, smoking, age, family history, and more. Although a majority of the conditions are largely preventable, there is still currently over 70 million Americans living with some form of CVD, which accounts for the significantly high rate of morbidity and the leading cause of mortality for men and women in the United States. Cerebral Vascular Accident (CVA), otherwise more commonly known as Stroke, is a subgroup of CVD. Stroke is a sudden onset of a neurologic deficit relating to an interruption of normal blood supply to any part of the brain resulting in some form of physiological deficit or death. According to the American Heart Association, stroke ranks as the number three leading cause of deaths behind Coronary Heart Disease (CHD) and cancer and is the number one cause of long term disability in the United States costing over $\$ 57.9$ billion in healthcare expenses. Each year approximately 700,000 individuals will experience a stroke (one every 45 seconds) (American Heart Association 2006).

Stroke is generally diagnosed as either ischemic (i.e. closure of a blood vessel) or hemorrhagic (i.e. bleeding from a blood vessel) in nature and immediate emergency treatment varies depending on the cause. Regardless of the origin, the downstream effects of stroke are general including impairments affecting the neuromuscular \& motor functions, vision \& hearing, mental status \& cognitive functions, sensory systems \& 
perception, and speech \& language capabilities; all corresponding to the location, severity, and timeframe of the injury within the brain. The neuromuscular deficit regularly includes paralysis or paresis of the side of the body contralateral to the lesion site within the brain. This is primarily because the majority of motor neurons originating on one side of the hemisphere that controls muscle movement crosses the midline of the body and innervates the muscles on the opposite side of the body. Injury to the descending neural pathways can result in abnormal regulation of the spinal motor neurons, causing a decreased ability to engage in voluntary movements resulting in conditions such as drop-foot which is a paralysis or weakness of the dorsiflexor muscles of the foot and.ankle, causing the dragging of the foot and toes during gait.

Because of the varying types of physical, mental, and cognitive deficits following a stroke incident, there is an array of rehabilitation techniques such as speech therapy, occupational therapy, physical therapy, and more. But one of the primary goals of all stroke rehabilitations is to focus on improving a patient's ability to sustain an independent lifestyle. This includes regaining their ability to perform basic Activities of Daily Living (ADL) such as ambulation. Recent investigation has found that an estimated $20 \%$ of stroke survivors will be confined to a wheelchair and $60 \%$ will have limited walking capabilities requiring the use of some form of walking aid (Robert et al. 2003). Even those who recover enough to become independent walkers, they will still exhibit abnormal walking patterns and are generally slower when compared to healthy subjects (Titianova el al. 2003). Therefore physical rehabilitation is essential in maximizing the level of recovery of a stroke survivor's physical capabilities. 
There is a strong consensus among rehabilitation experts that the most important element in any rehabilitation program is carefully directed and motivated exercises, wellfocused with active participation, and repetitive practice; the same type of training used by all people when they learn a new skill, such as playing the piano or pitching a baseball. The goal of rehabilitation therapy is to help maximize the level of physical recovery in the shortest amount of time spent in therapy. Physical rehabilitation focuses on regaining the use of stroke-impaired limbs, teaches compensatory strategies to reduce the effect of remaining deficits, and establishes an ongoing exercise programs to help people retain their newly learned skills. The most common method of physical rehabilitation training used is based on theories originating in the 70's (Brunnstrom 1970, Bobath 1978). Their focus is on the compensatory training of the sound side in cooperation with maintaining muscle strength, Range of Motion (ROM), and reduction of spacticity. The exercises are performed under the close supervision of a therapist using basic equipments (i.e. pulley, chairs, sticks, etc). This form of therapy is commonly used as the benchmark for gauging physical recovery in stroke rehabilitation.

Although these traditional techniques have shown to be beneficial in the recovery of stroke patients, newer and more innovative techniques have seemingly emerged which provide more dynamic and task-oriented approaches to stroke rehabilitation. Treadmill training for example provides the benefit of a task specific exercise. This exercise allows patients to perform the exact walking motion they are attempting to regain on a stationary treadmill with the assistance of physical therapists and/or a body weight support (BWS) harness. The therapists will manually position the patient's foot and legs during the gait exercise to simulate correct walking patterns (Mauritz 2004). Study into the use of 
robotics for stroke rehabilitation has also shown promising results. Patients utilizing the MIT-MANUS device which provides robotic assistance in the completion of specific elbow and shoulder exercises showed greater improvement in motor control than the control group who did not receive any robotic aid (Fasoli et al. 2003). The use of Electromyography (EMG) Biofeedback (BFB) has shown to help improve voluntary motor control by providing an alternative sensory input to motor control to compensate for those lost sensory inputs related to the injury (Basmajian et al. 1975).

The specific purpose of this research thesis is to develop a new robotic AnkleFoot Orthosis (AFO) utilizing a closed-loop biofeedback control system focused on improving neuromuscular control and gait symmetry of the ankle motion for stroke patients suffering from drop-foot. The device will serve as a training tool requiring active participation by the user in stationary and active training exercises. The overall goal is to present a new rehabilitation technique aimed at providing a higher level of neurological and physical recovery resulting in a more independent and natural walking motion for the patient. 


\section{BACKGROUND and SIGNIFICANCE}

\subsection{THE CENTRAL MOTOR SYSTEM}

\subsubsection{Anatomical Components}

Human motion is achieved through a synergy of two physiological systems of the human body consisting of the nervous system and the musculoskeletal system. The anatomical components responsible for motion begin with the Central Nervous System (CNS) which includes specific regions within the cerebral cortex (e.g. premotor cortex, primary motor cortex, primary sensory cortex), the basal nuclei, the cerebellum, the medulla oblongata, the thalamus, the mesencephalon, and the spinal cord. Figure 2.1 displays some of the anatomical components within the CNS which play a part in human motion. The cerebral cortex is the outermost layer of the cerebrum and plays a central role in complex brain functions including processing of somatic sensory information and regulation of skeletal muscle contractions. The basal nuclei (i.e. basal ganglia) provides general movement patterns for voluntary motor activities as well as rhythmic movement cycles like walking of skeletal muscle at the subconscious level. The cerebellum is an automatic integration center specialized in processing sensory information, provides control into the postural muscles, and coordinates the execution of voluntary and involuntary movements. The movement patterns generated by the cerebellum are acquired starting from infancy by learning through trial and error and repetitive practice. The medulla oblongata (i.e. medulla) is the junction between the brain and spinal cord 
and plays an important role in relaying sensory information to the brain. The thalamus directs afferent sensory information to the basal nuclei and to specific locations within

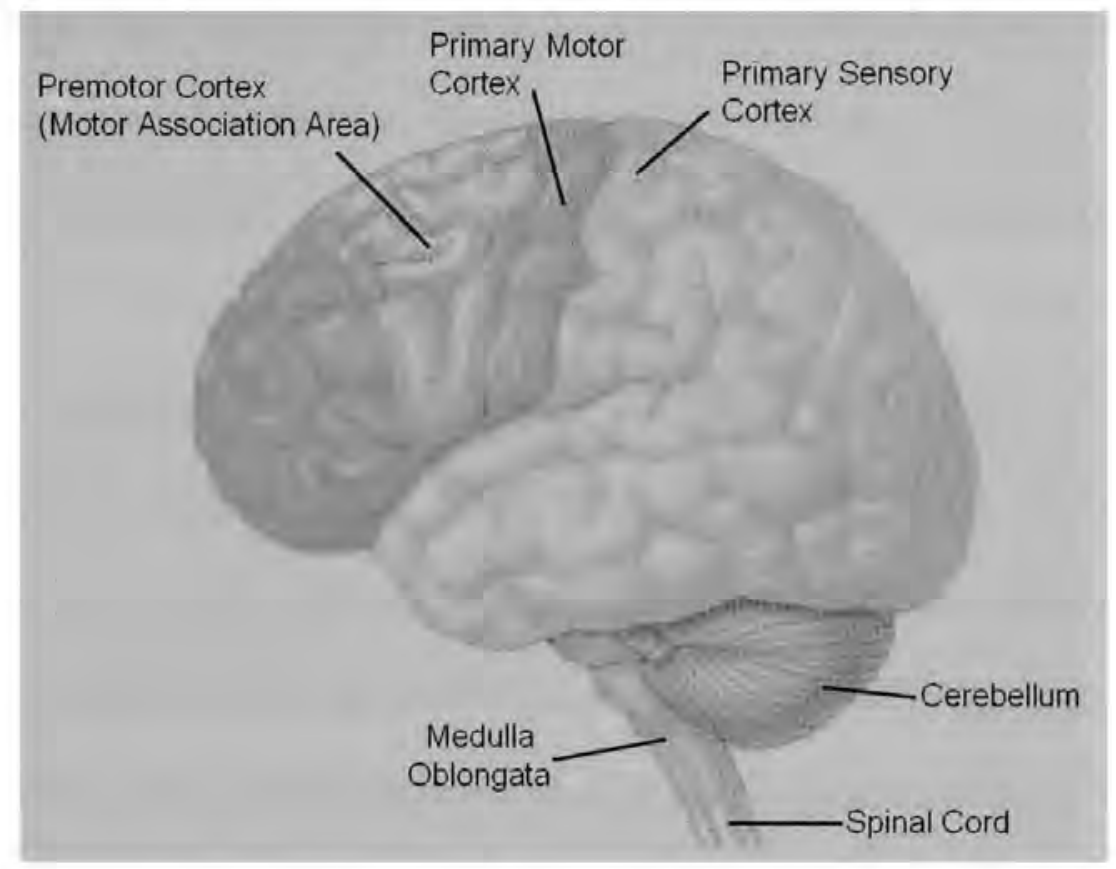

Figure 2.1 Lateral view of the brain with selected anatomical components within the CNS responsible for motion (Martini et al. 2003)

the primary sensory cortex of the cerebral cortex. The mesencephalon (i.e. midbrain) receives visual sensory input and relays the information to the cerebellum. Finally the spinal cord is a tubular structure protected within the bony vertebral column whose primary function is the transmission of neural inputs between the brain and peripheral anatomy (Martini et al. 2003).

The next set of anatomical components within the nervous system includes the Peripheral Nervous System (PNS). The PNS consisting of the neurons that reside outside the CNS which includes the Somatic Nervous System (SNS). The SNS contains somatic motor neurons whose primary function is the voluntary control of body movements through the innervation of skeletal muscles. The somatic motor neurons have single 
axons originating from the CNS which carry the excitatory signals to the target skeletal muscles (Silverthorn 2004). The somatic motor neurons branches and attaches to the target muscles at the neuromuscular junction. Chemical synapses at the neuromuscular junction create action potentials that triggers muscle contraction.

The musculoskeletal system is the second organ system responsible for human movement. It is composed of proprioceptors, skeletal muscles, bones, joints, and tendons. The proprioception receptors include the golgi tendon organs, muscle spindle, and joint capsules located within the muscles and joints. They provide sensory information to the brain as to the position and movement of skeletal muscles and joints relative to one another without the need of visual cues. The remaining components of the musculoskeletal system serve to provide support, protection, and movement to the human body.

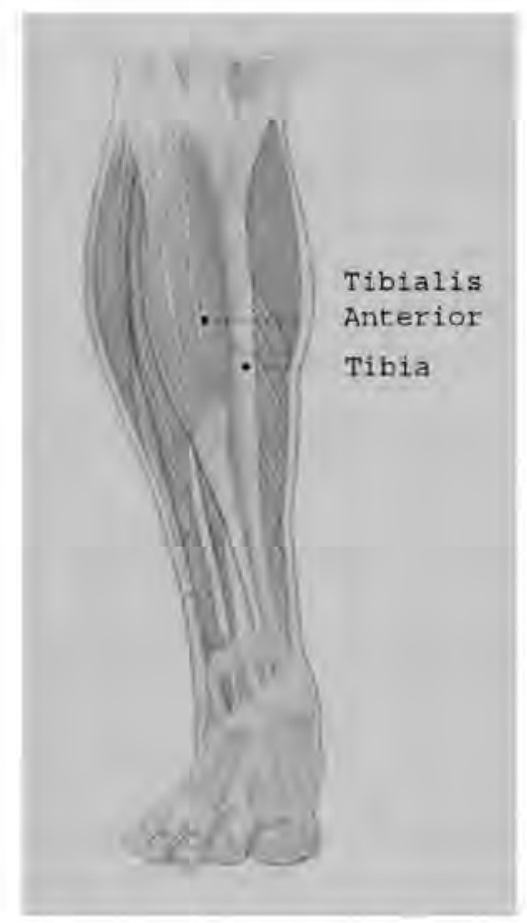

Figure 2.2 Anterior view of the tibialis anterior muscles of the right leg (Martini, 2003) 
.The tibialis anterior muscle is located in the lower leg proximal to the tibia (Figure 2.2) and is the primary muscle responsible for dorsiflexion of the ankle. The muscle is innervated by the deep fibular nerve of the SNS (Martini et al. 2003).

\subsubsection{Sensory Pathways and Motor Pathways}

In order for the human body to successfully achieve coordinated movements, it must first have the ability to establish its own relative position in space and secondly have the ability to execute the processes necessary to generate motion. This requires an intricate and highly integrated system of neural pathways composed of sensory and motor neurons connected to the CNS. The sensory pathways provide a network for the body to transmit afferent sensations gathered from its peripheral receptors to the CNS where it can then orient the body's position in space. The motor pathways allow the CNS to send efferent signals to the skeletal muscles to produce the desired motion. There is a continuous exchange of information between the CNS, sensory pathways, and motor pathways.

The CNS relies heavily on three forms of sensory inputs which are proprioceptive, vestibular, and visual sensation to help the CNS monitors the body's position in space before and during a movement sequence. Proprioceptive receptors terminate at both the primary sensory cortex as well as the cerebellum. Receptors which end at the cerebral cortex travel up axons along the posterior column pathway which crosses the body's midline at the medulla. Whereas the axons that synapse to the cerebellum traverse the spinal cord via the spinocerebellar pathway (Martini et al. 2003). Sensory information acquired from the vestibular apparatus and the otolith organs of the 
inner ear provides the body with a sense of equilibrium, balance, as well as the position and acceleration of the head in space. Information is carried along the vestibulaocochlear nerve directed to both the cerebellum and the vestibular nucleus within the medulla (Silverthorn 2004). Visual cues provide sensory information on stable spatial features that helps establish the body's relative position in space. For example, we use the horizon as a spatial landmark to orient us in relation to the ground. These visual cues travel along the optic nerve and synapse onto the thalamus where collateral pathways relay the information to the mesencephalon where it is processed.

The motor pathways are responsible for transmitting signals to the skeletal muscles to initiate movement based on conscious voluntary commands, subconscious commands, and in response to sensory feedback. The efferent signals are carried over three integrated pathways which are the corticospinal pathway, the medial pathway, and the lateral pathway. The corticospinal pathway primarily controls voluntary movement whereas the medial and lateral pathways provide subconscious muscular control. In addition, the activities within the three motor pathways are further monitored and adjusted at the subconscious level by signals provided from the basal nuclei and cerebellum (Martini et al. 2003).

The corticospinal pathway (i.e. pyramidal system) is a large collection of motor neurons that provides a direct connection between the cerebral cortex and skeletal muscles (Figure 2.3). Somatic motor neurons originating in the primary motor cortex extend through the brain stem and spinal cord where they synapse onto lower motor neurons which innervate the skeletal muscles. Approximately $85 \%$ of the axons in the corticospinal tract cross the midline of the body (decussate) at the pyramids located within the medulla. This 
attributes to the contralateral muscular control between opposite sides of the brain hemisphere and body.

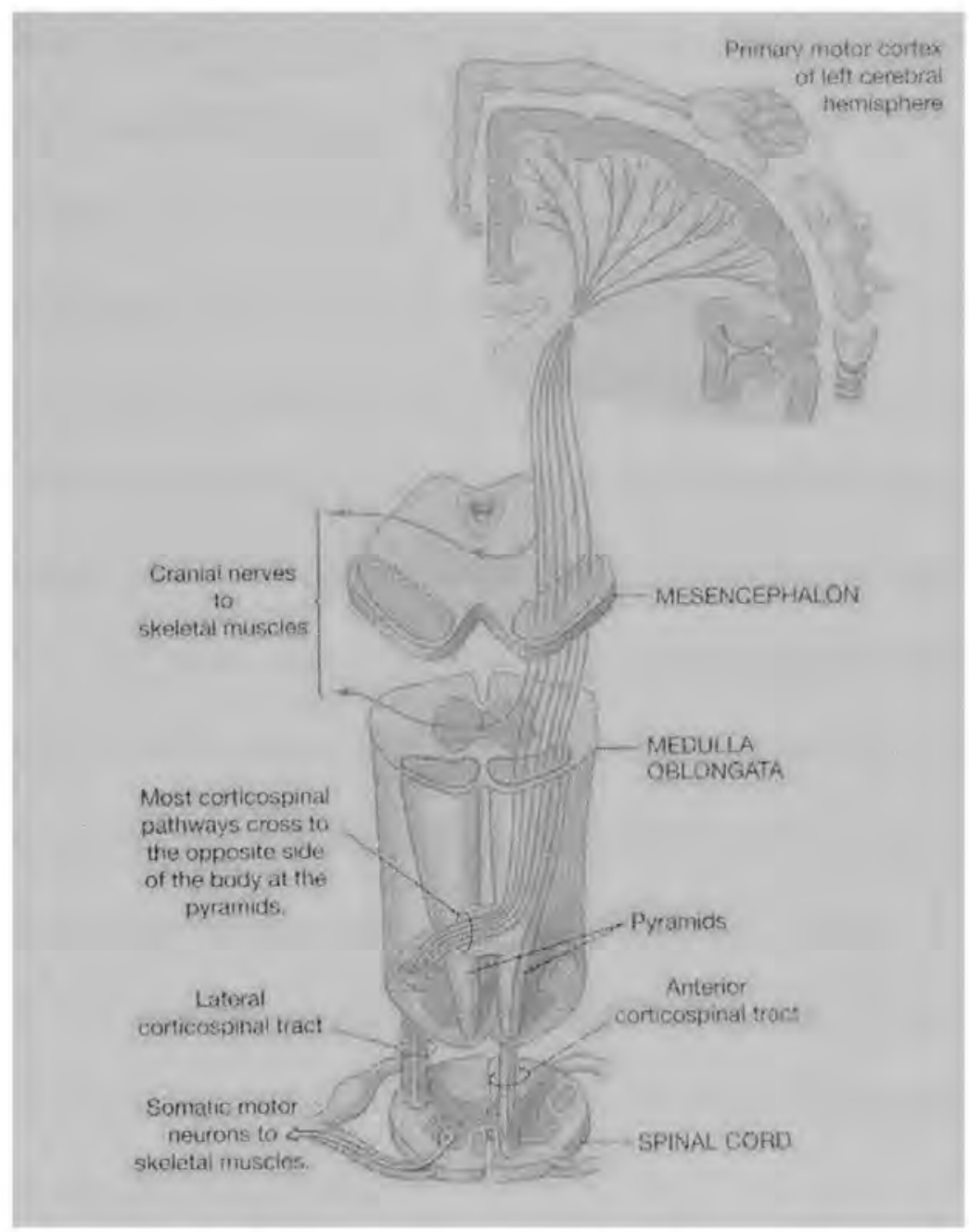

Figure 2.3 Corticospinal Pathway (Martini et al. 2003)

The medial and lateral pathways helps modify the motions transmitted through the corticospinal pathway on a subconscious level by stimulating or inhibiting the same lower motor neurons as those used by the corticospinal pathway. The medial pathway has three tracts which carry signals originating form the brainstem (i.e. vestibulospinal tract, tectospinal tract, and reticulospinal tract). The vestibular nuclei located within the medulla responds to changes in orientation of the head and will issue motor commands to 
maintain posture and balance. The axons conduct through the vestibulospinal tract located within the spinal cord. The superior and inferior colliculus nuclei located within the mesencephalon will issue motors commands that descend through the tectospinal tract based on visual and auditory changes. Finally the reticular formation located within the brain stem transmits motor commands through the reticulospinal tract. The lateral pathway also contributes to the control of muscle tone. The red nuclei located within the mesencephalon will issue involuntary motor commands to help maintain muscle tone and limb position. The axons descend through the rubrospinal tract within the spinal cord.

The basal nuclei and cerebellum provides an additional level of control within the motor pathways. The basal nuclei has two major neural pathways which it uses to influence the activity in the motor pathway. The first is a set of axons which synapses onto the thalamic neuron and is then relayed to the premotor cortex. The second pathway consists of neurons that synapse directly to the reticular formation. The cerebellum contains neural networks that connect to the cerebral cortex, basal nuclei, and motor centers in the brain stem to influence their activity on the corticospinal, medial, and lateral pathways (Martini et al. 2003).

\subsubsection{Coordination of Body Movements}

Human motion can be loosely classified as either a reflex, voluntary, or rhythmic movement. The distinction between each type of movement is not always well-defined therefore particular movements can be categorized as a combination of the three classifications. For example reflex motions can be influenced by voluntary control and even voluntary movements can become reflexes with extensive training. 
A reflex movement is an involuntary movement triggered by a stimulus. Postural reflexes (e.g. walking or pitching a baseball) allow our body to remain in a state of balance as we move through space by providing the ability to move our body's center of mass in a controlled manner in relation to the base of support (Nichols 1997). Postural reflexes continually monitor and adjust our bodies muscle tone and position (i.e. head, neck, trunk, and limbs) based on stimulus received from the visual and vestibular sensory inputs, as well as proprioception receptors (Silverthorn 2004). The sensory information is integrated and processed within the brain stem (i.e. mesencephalon and medulla oblongata) and cerebellum. The necessary motor commands are then issued at a subconscious level by these systems and carried along the various motor pathways to the appropriate target destination (e.g. skeletal muscles). This provides automatic adjustments without conscious effort. These coordinated movements are significantly influenced by prior experience and practice and are essential in both voluntary and rhythmic movements.

The process of achieving voluntary motion consists of three stages: (1) the planning stage, (2) the initiation of movement, (3) and the execution of movement. Figure 2.4 provides an illustration on the general sequence of events that occur within the CNS associated with voluntary motion. During the planning stage a decision in made to perform a specific motion. A strategy is generated in the frontal lobes of the cerebral cortex and relayed to the premotor cortex. This information is then shared with the basal nuclei and the cerebellum through neural interconnections. The objective is to generate a movement strategy that best achieves the goal of the intended motion. 


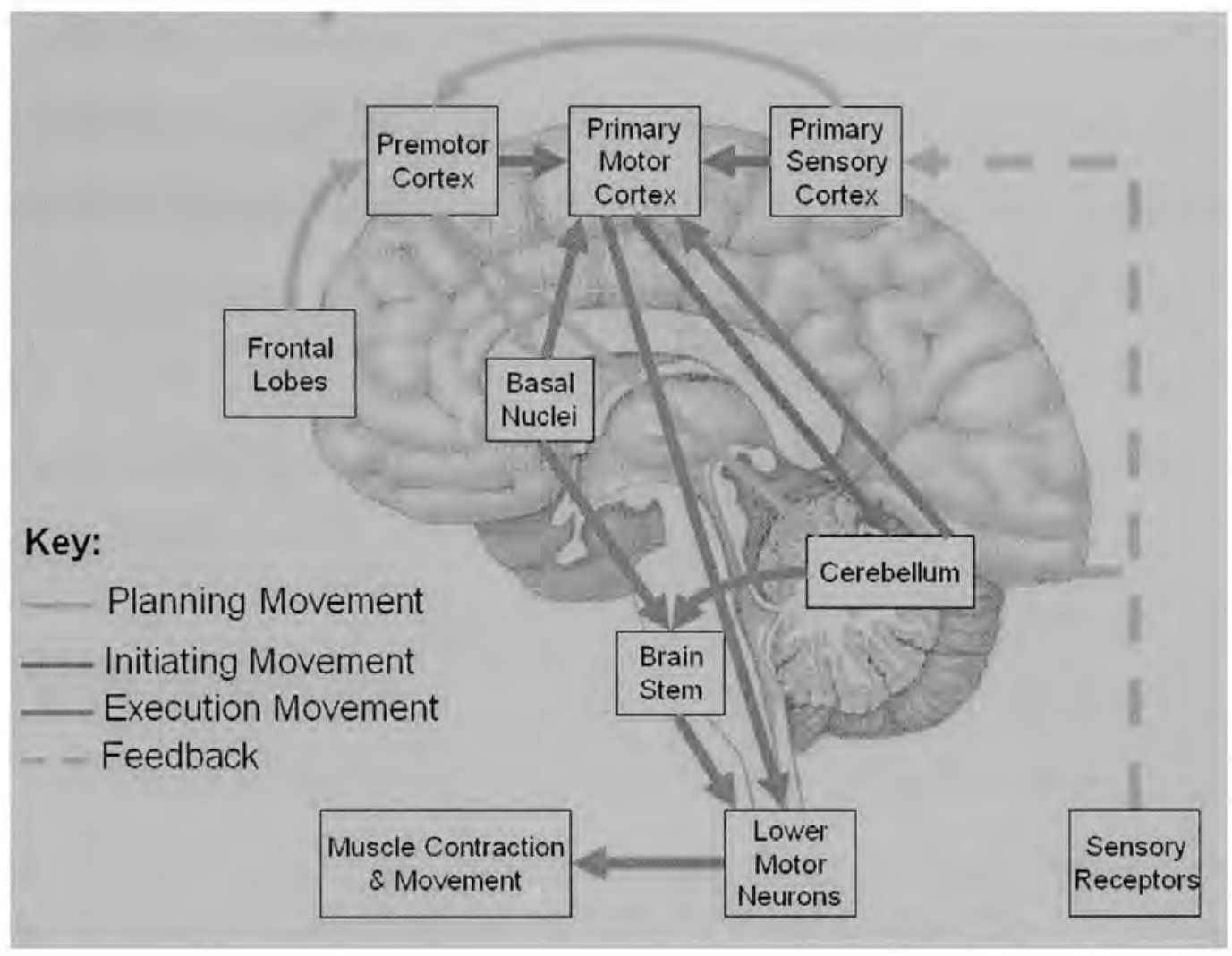

Figure 2.4 Control sequence for voluntary movements within the CNS (Martini et al. 2003)

Once the strategy has been established, the movement is initiated by a signal generated from the premotor cortex to the primary motor cortex that is also relayed to the cerebellum. This process is focused at establishing the sequence and timing of muscular contractions necessary to perform the desired motion. To carry out the execution of the movement, action potentials are generated by the primary motor cortex that is carried by the corticospinal tract to the target skeletal muscles. In parallel, the cerebellum continually integrates sensory feedback received through the sensory pathways and automatically adjusts postural muscles and fine tunes the necessary coordinated movements. In addition the basal nuclei also provide adjustments to posture, balance, and gait in response to the voluntary motion. 
Rhythmic movements include actions such as walking and running and are achieved through a combination of voluntary and reflex movements. They require a voluntary effort by the cerebral cortex to initiate and terminate the action. Once initiated, there are central pattern generators within the interneurons of the spinal cord. They provide a similar mechanism as a reflex which allows the body to maintain the coordinated sequence of muscle movements without conscious input from the brain (Silverthorn 2004).

\subsection{HUMAN GAIT}

Walking is the most common form of gait and is defined as a means of bipedal locomotion utilizing the legs for movement. Under normal conditions, walking provides an efficient means of transportation requiring low energy expenditure and exerting minimal strain on the body (Perry 1992). The action encompasses a repetitive sequence of limb motions that allow forward progression of the body while maintaining balance and stability. The model for human walking is based on an inverted pendulum design where a complete sequence of functions by one limb is defined as a Gait Cycle (GC).

\subsubsection{Gait Cycle}

A complete GC is also referred to as a stride and is generally divided into two periods which are referred to as the stance period and swing period. The stance periods defines the timeframe during which the foot is in contact with the ground to support the weight of the individual while the body is advanced in the forward direction. The swing period signifies the duration when the foot is lifted off the ground for limb advancement. 
The distribution of time within each period during a typical walking GC is generally defined as $60 \%$ stance and $40 \%$ swing.

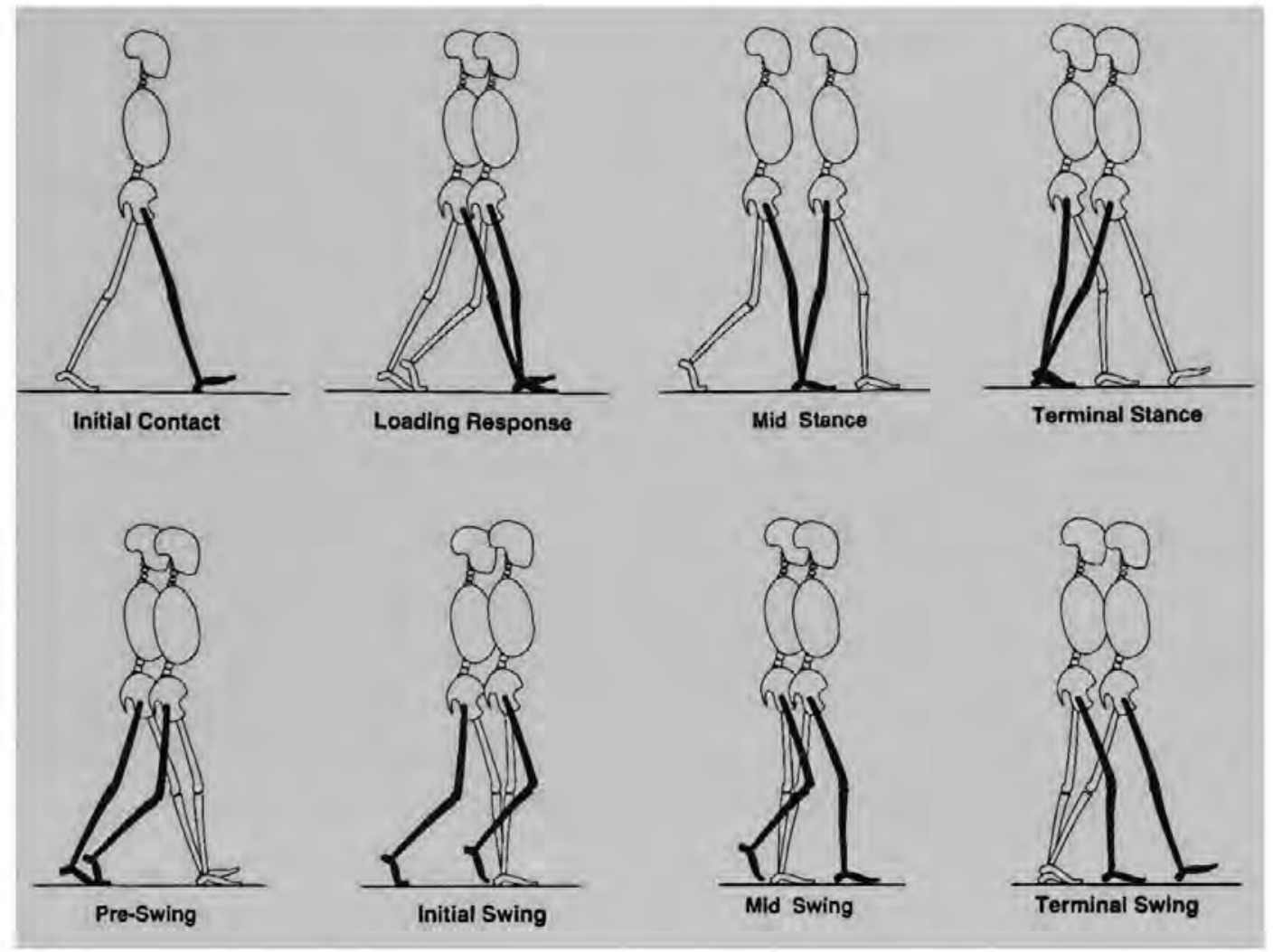

Figure 2.5 Stick figure illustration of the individual phases within a gait cycle for the right limb (Perry 1992)

Each stride is further subdivided into eight phases, each of which exhibits a motion patterns that perform a different functional task. The eight phases are (1) Initial Contact, (2) Loading Response, (3) Mid Stance, (4) Terminal Stance, (5) Pre-Swing, (6) Initial Swing, (7) Mid Swing, (8) Terminal Swing. Figure 2.5 provides an illustration of the individual phases. Table 2.1 provides a summary description of each phase and its relation to the GC. 


\begin{tabular}{|c|l|l|c|}
\hline Period & Phase & \multicolumn{1}{|c|}{ Description } & $\begin{array}{c}\text { \% of Gait } \\
\text { Cycle }\end{array}$ \\
\hline \multirow{5}{*}{ Stance } & $\begin{array}{l}\text { Initial } \\
\text { Contact }\end{array}$ & $\begin{array}{l}\text { Includes the moment when the target foot touches } \\
\text { the floor }\end{array}$ & $0-2 \%$ \\
\cline { 2 - 4 } & $\begin{array}{l}\text { Loading } \\
\text { Response }\end{array}$ & $\begin{array}{l}\text { Encompasses the initial floor contact until the } \\
\text { opposite foot is lifted off the ground for swing }\end{array}$ & $0-10 \%$ \\
\cline { 2 - 4 } & Stance & $\begin{array}{l}\text { Begins with the swing of the opposite foot and } \\
\text { continues until the body is aligned over the target } \\
\text { forefoot }\end{array}$ & $10-30 \%$ \\
\cline { 2 - 4 } & $\begin{array}{l}\text { Terminal } \\
\text { Stance }\end{array}$ & $\begin{array}{l}\text { Includes when the heel of the target foot raises off } \\
\text { the ground until the opposite foot strikes the } \\
\text { ground }\end{array}$ & $30-50 \%$ \\
\cline { 2 - 4 } & $\begin{array}{l}\text { Pre- } \\
\text { Swing }\end{array}$ & $\begin{array}{l}\text { Continues with the target foot heel raising and ends } \\
\text { when the foot is lifted off the ground }\end{array}$ & $50-60 \%$ \\
\hline \multirow{5}{*}{ Swing } & $\begin{array}{l}\text { Initial } \\
\text { Swing }\end{array}$ & $\begin{array}{l}\text { Begins when the target foot is lifted off the ground } \\
\text { during swing until it is opposite the stance leg }\end{array}$ & $60-73 \%$ \\
\cline { 2 - 5 } & $\begin{array}{l}\text { Mid } \\
\text { Swing }\end{array}$ & $\begin{array}{l}\text { The target foot continues swing past the opposite } \\
\text { stance leg until the target leg is forward of the } \\
\text { body and the tibia is in a vertical position }\end{array}$ & $73-87 \%$ \\
\cline { 2 - 4 } & $\begin{array}{l}\text { Terminal } \\
\text { Swing }\end{array}$ & $\begin{array}{l}\text { Encompasses when the tibia of the target leg is } \\
\text { past vertical and continues until the foot strikes the } \\
\text { ground }\end{array}$ & $87-100 \%$ \\
\hline
\end{tabular}

Table 2.1 Summary Description of the Phases within a Gait Cycle

\subsubsection{Ankle-Foot Complex}

The ankle is considered to be the primary site of all leg-foot interaction. It plays an important role in gait in providing shock absorption and allowing a smooth progression within the stance and swing periods. The motion of the ankle achieved during gait is defined as either a dorsiflexion, signifying an upward travel of the foot, or a plantar flexion, which is the downward movement of the foot relative to the leg. The range of motion the ankle follows during a normal walking $\mathrm{GC}$ follows a general pattern for all individuals (Murray 1964). This pattern is composed of four motion arcs which transition between plantar flexion and dorsiflexion (Figure 2.6). A neutral position is 
usually defined as a right angle between the foot and tibia, similar to an upright standing position.

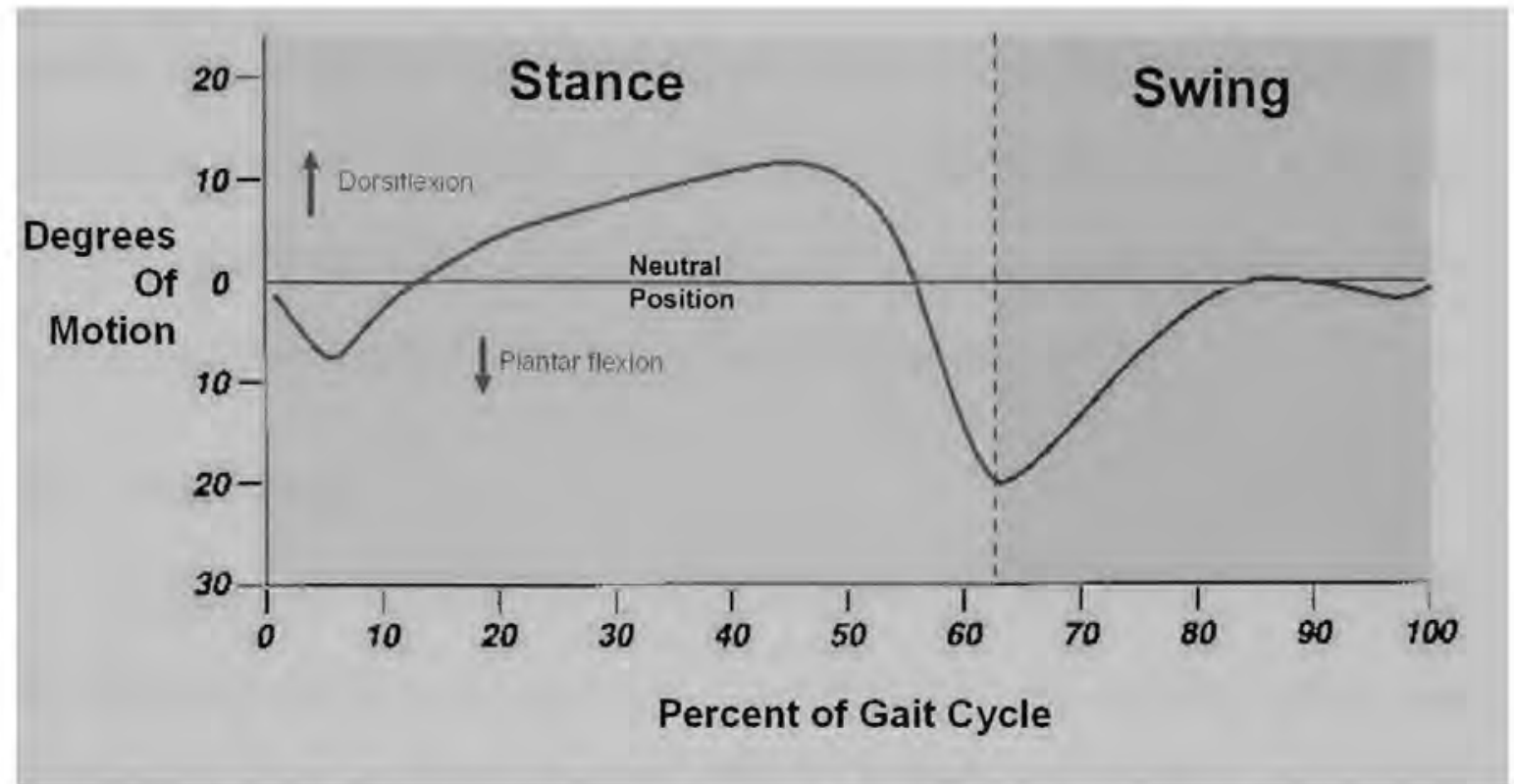

Figure 2.6 Mean Ankle Range of Motion during Gait Cycle (Perry 1992)

The entire ankle motion during walking usually has a complete ROM of approximately $30^{\circ}$ with a typical boundary range of $10^{\circ}$ doriflexion past neutral occurring during Termial Stance and a $20^{\circ}$ plantar flexion past neural during Pre-Swing. During the swing phase, the only motion that occurs is the final dorsiflexion of the ankle back to the neutral position. This allows for proper foot clearance from the ground and aligns the foot in the optimal position for heel strike at the initial contact phase.

\subsection{STROKE}

A Stroke, or Cerebroascular Accidents (CVA), is defined as a sudden onset of a neurologic deficit relating to a disruption of blood supply to the brain and should be treated as a medical emergency. The disruption can either be ischemic (i.e. closure of a blood vessel) or hemorrhagic (i.e. bleeding from a blood vessel) in origin resulting in 
temporary or permanent damage to the brain tissue (Singleton 1987, Kaplan et al. 1986). In an ischemic stroke, the reduced blood supply is attributed to either the formation of a thrombus or an embolus which deprives the brain tissue of oxygen and glucose in addition to preventing the removal of toxic metabolites which causes cellular elements to die. In a hemorrhagic stoke, a ruptured or bleeding blood vessels causes blood to flow directly into the brain tissue or surrounding areas causing tissue damage.

\subsubsection{Risk Factors}

There are several risk factors associated with the occurrence of stroke. Some of the risks factors cannot be changed such as a person's age, their heredity, gender, and prior history (American Heart Association 2007). 95\% of strokes occur in people who are of the age 45 and older, and two-thirds of strokes occur in those over the age of 65 (Senelick R. et al. 1994, NINDS 1999). Men have a 1.25 times higher risk of developing a stroke (NINDS 1999) but women are 60\% more likely to suffer a fatal stroke due to the older age at which stroke occurs (Villarosa 1993). But the majority of common risk factors including high blood pressure, smoking, diabetes, high blood cholesterol, poor dieting, and physical inactivity can be treated, changed or controlled. Lowing high blood pressure levels can dramatically reduce the risk of vascular complications and overall mortality (Neal et al 2000, Staessen et al. 2001). A separate research study indicated that individuals who smoked doubled their risk of having an ischemic stroke (Shinton et al 1989). In general studies indicate that reducing hypertension, treating diabetes properly, lowering blood cholesterol levels, cessation of smoking, healthy dieting, regular exercise, 
and avoidance of illicit drugs and excessive alcohol consumption all have a significant contribution to preventing the occurrence of a stroke (Hack et al. 2003)

\subsubsection{Neuromuscular Effects of Stroke}

The term neuromuscular relates to any activity involving or affecting the nervous and muscular elements. The neurological signs and symptoms following an onset of stroke can include changes in a person's mental status, motor function, sensation, vision $\&$ audition, and language. The deficits vary widely based on the size and location of the lesion (Stanford Hospital \& Clinic 2007). Lesion to the central brain (i.e. thalamus, basal ganglia, and internal capsule) results in greater motor deficit than damage to the surrounding neocortical area. Injury to non-primary motor neurons still resulted in muscle weakness (Singleton et al. 1987). Damage incurred to the motor cortex and descending tracts affect muscle force production because there is a decrease in total EMG activity (Tang 1981, Trombly 1985). This alters the number of motor unit recruited, the type of motor unit recruited, and the motor unit discharge frequency (McComas 1973, Mayer et al. 1980, Rosenfalck et al. 1980). If the rate of firing is diminished sufficiently enough, the chain of twitches can fail to fuse into a strong contraction (Rosenfalck 1980). Weakness from these sources are further compounded by the changes in the property of the motor unit and morphological changes in the muscle which occur adaptively as a consequence of denervation, decrease in physical activity, and general disuse (Singleton et al. 1987, Shepherd 2001). Muscle weakness and disordered motor control combine to result in functional movement disability. 


\subsubsection{Cortical Reorganization and Motor Learning}

It was proposed that functional learning of the neural circuitry achieved during development is similar with adult learning suggesting that training can induce longlasting neural changes (Karni 1996). Plastic neuronal changes were induced following relevant activities and rewarded behavior (Liepert et al. 1998). Research also showed that repeated practice is important to motor recovery in hemiplegic patients (Bütefisch et al. 1995). Therefore modern concepts of motor learning have shifted from conventional neurodevelopment to a more dynamic and task oriented approach (Barbeau et al. 2001) focusing on various mechanisms involved with locomotor recovery, including the paradigm that the training program must be locomotor specific (Lovely et al. 1986). The new intervention strategies are placing more emphasis on active exercise and task specific training while still including active and passive methods of preserving muscle extensibility (Shepherd 2001). Training has the potential to promote brain reorganization and to optimize functional performance. Although rehabilitation services have been generally slow to adopt these new attitudes and methodologies, therapists are beginning to rely less on one-to-one, hands-on service delivery and have been integrating new technological advances in training equipment which increase the time the patient spends doing active exercises aimed at increasing muscular strength, control, skill, endurance, and fitness.

\subsection{GAIT REHABILITATION TREATMENTS FOR STROKE}

There currently is no standard single treatment for stroke patients because each patient is unique with different problems, potentials, and abilities (Bobath 1977). But 
research has shown that proper rehabilitation programs following an onset of stroke do provide patients with a greater level of improvement than spontaneous recovery alone (Johnstone1987). A study was conducted of 114 stroke patients to see how rehabilitation affected functional gains and the ability to live independently. After participating in the rehabilitation programs, significant statistical gains were seen in all the functional categories including mobility, walking, and transfer activities. Prior to the research 54\% of the patients were confined to nursing homes and hospitals for care. At the conclusion of the program, $73 \%$ were living independently at home, with a spouse, or alone (Lehmann et al 1975).

Neuromuscular disorders affecting the lower limbs such as hemiparesis will impair normal gait functions resulting in higher energy expenditure, delayed ambulation speed, and additional physiological pathologies (Johnstone1987). It has been found that approximately $20 \%$ of stroke survivors will be confined to a wheelchair, and approximately $60 \%$ will have limited walking capabilities (Robert 2003). Traditional gait rehabilitation has been focused on treating the effects of spasticity and abnormal reflexes (Westcott 1967) using fairly basic general movements. The Neurodevelopment Technique (NDT) established by Bobath (Bobath 1978) devotes a considerable amount of rehabilitation training on inhibiting spasticity and other abnormal responses because he assumes that abnormal postural reflex activity is the primary cause of movement disorders. 


\subsubsection{Treadmill Training}

The practice of utilizing a treadmill machine to facilitate gait re-education has shown to provide marked recovery benefits in stoke patients. A study was conducted on five chronic stroke patients ranging from 9-70 months post stroke (Silver et al. 2000). The rehabilitation program consisted of walking on a treadmill three times per week for three months duration. The intensity of the exercise was based on the individual's tolerance for completing a 40 minutes program at $60-70 \%$ of their maximum heart rate. The validation for the program was to evaluate the time necessary to complete an exercise which required the patient to arise form a chair, walk a straight distance of $3.1 \mathrm{~m}$ without the use of their normal walking aid, and return to a separate chair positioned at the end of the walkway. The time needed to complete the entire exercise decreases by $21 \%$ after the completion of the training period. The individuals exhibited an increase in velocity and cadence as well as a decreased mean stance and swing duration.

Prior animal studies using spinal cats have shown that an adult spinal cord can produce near-normal walking patterns after a period of interactive locomotive training on a treadmill with partial body weight support provided (Barbeau et al. 1987). This type of study led to the development of a Body-Weight Support (BWS) harness to be used in conjunction with treadmill training for stroke patients which has expanded the rehabilitation program for patients with more severe ambulatory handicaps (Figure 2.7).

In a study of seven nonambulatory hemiparetic patients, BWS treadmill training was shown to be more effective than traditional physiotherapy using the Bobath methods (Hesse et al. 1995a). The study was structured in an A-B-A arrangement case study. The A-phase included 30 minutes of daily treadmill training with BWS for three weeks. 
While the B-Phase was based on 45 minutes of traditional physiotherapy for three weeks.

The gait parameters for the experiment only improved during the A-Phase.

In a separate study comparing BWS to no-BWS treadmill training, a group of 79 hemiparetic patients with abnormal gait patterns ranging from 27-148 days post stroke were evaluated (Visintin et al. 1998). The program consisted of a 20 minute session on the treadmill four times a week for a total of six weeks. The BWS group were provided with up to $40 \%$ BWS at the start of the program and progressively decreased as the patients gait patterns improved. Although both groups showed marked improvements in balance, motor recovery, walking speed, and endurance, the BWS group had slightly higher scores in walking speed at the three-month follow-up assessment.

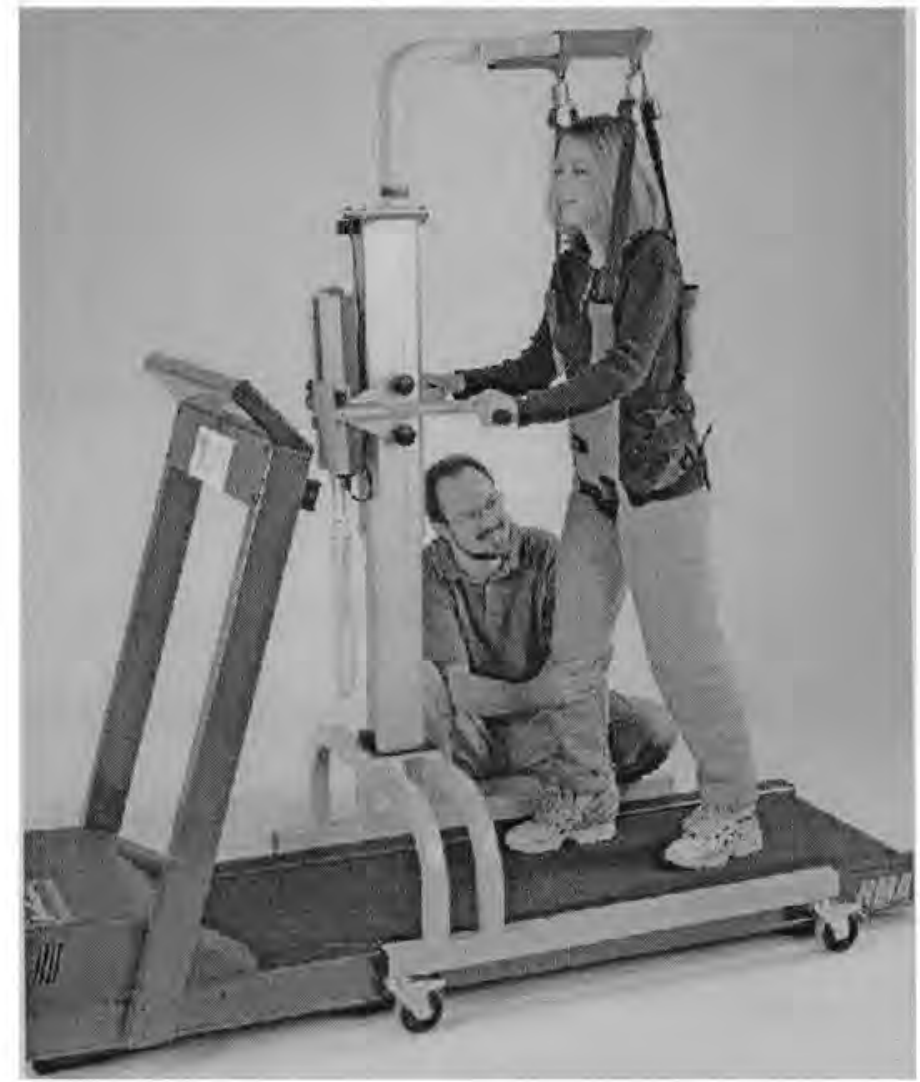

Figure 2.7 Body Weight Support Treadmill Training 


\subsubsection{Robotic-Assisted Therapy}

The use of robotic technology in the rehabilitation services is emerging as an invaluable tool for treating individuals suffering from physical disability. Robot-assisted therapy permits semi-autonomous practice of therapeutic tasks and have shown to improve motor functions in stroke patients. The MIT-MANUS is a 2 Degree-of-Freedom (DOF) upper limb neurological training tool developed for clinical neurological applications (Figure 2.8) (Hogan 1995).

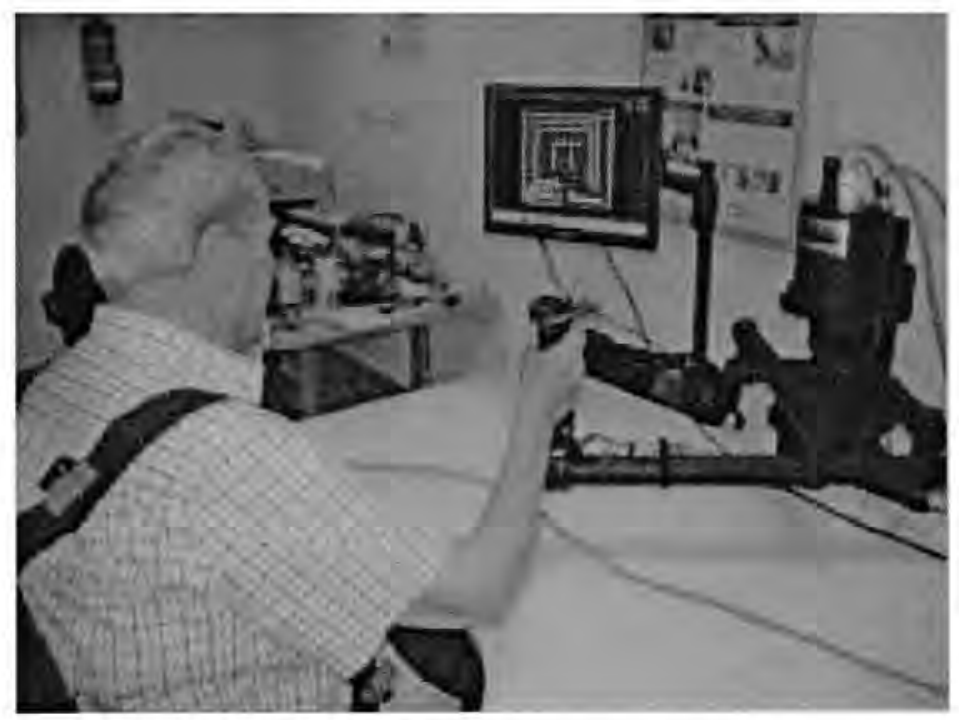

Figure 2.8 MIT-MANUS

The MIT-MANUS can guide or perturb the movement of a subjects arm while providing visual feedback and recording the motion and mechanical quantities.

A clinical trial was conducted using the MIT-MANUS (Krebs 1998). The research included 20 hemiparetic stroke patients in a double blinded study. The population was divided into two equal groups. Both groups received conventional therapy. The experimental group also received 4-5 hours/week of training using the MIT-MANUS for 7 weeks which would provide automated assistance to the user if they 
were unable to complete the movement. The control group only received 1 hour/week of training; however, the MIT-MANUS would not assist the subject in the motion. The user had to rely on the unimpaired limb to help complete the motion if necessary. The results showed that the experimental group had statistically higher motor improvements in the shoulder and elbow in the Fugl-Meyer, motor power, and motor status scores when compared to the control group. In a similar clinical study, 20 chronic stroke subjects were put through a four week training program using the MIT-MANUS (Fasoli et al. 2003). The subjects ranged form 1-5 years post stroke and participated in three 1 hour session per week for a six week training period. The groups received either a sensorimotor program which consisted of the MIT-MANUS assisting the user to complete the upper limb motion; or they were placed into a progressive-resistance group where they performed the same goal oriented movements with the MIT-MANUS providing an opposing force. The resistive force was generated using an adaptive algorithm. The results again showed a reduction of motor impairment of the hemiparetic limb. The assessment scored showed statistically significant numbers for the Fugl-Meyer test $(\mathrm{P}=0.001)$, motor status scale $(\mathrm{P}=0.01)$, and motor power $(\mathrm{P}=0.0001)$ for the shoulder and elbow. The motor improvements were sustained over a 3-year period after the hospital discharge (Volpe et al. 1999)

\subsubsection{Electromyography Biofeedback}

The use of electromyography biofeedback (EMG BFB) for rehabilitation has seen increasing growth in the past several decades. It utilizes surface electrodes which monitors muscle contraction and converts the information into a visual and/or audio 
output (Figure 2.9). This form of treatment provides a noninvasive method for conveying information to a patient in real-time in order to raise their awareness and conscious control of the related physiological activities. By providing access to physiological information about which the user is generally unaware due to injury, biofeedback allows users to gain control over physical processes previously considered automatic. Research has shown EMG BFB to be a plausible adjunct to other forms of stroke rehabilitation.

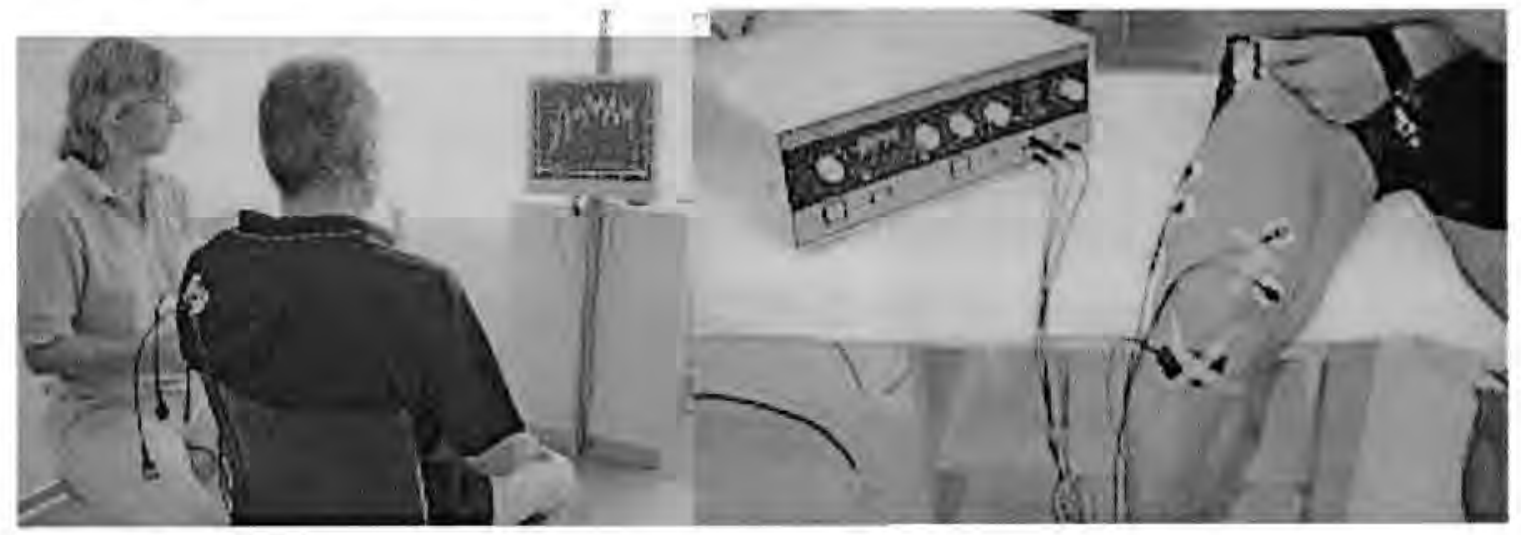

Figure 2.9 Electromyography Biofeedback Training

EMG BFB has been used to successfully improve the conditions associated with drop-foot. A clinical trial of 20 adult stroke patients exhibiting residual foot dorsiflexion paresis was conducted (Basmajian et al. 1975). The patients were randomly divided into two groups of ten patients each. The control group received traditional therapeutic exercises for 40 minutes a day for five weeks. The experimental group received 20 minutes of therapeutic exercise and 20 minutes of biofeedback training for the same five week duration. After the completion of the program, an evaluation into the patient's strength and ankle ROM showed that both groups had improvements in both categories. However, the experimental group had results that were approximately twice as great as the control group. In a related study, a group of 15 stroke patients with residual foot 
dorsiflexion paresis ranging from 4-120 months post onset was selected for gait analysis (Takebe et al. 1976). The groups were randomly divided into three groups. The first group of six patients received 40 minutes of conventional therapeutic exercise three times a week for five weeks. The second group of six patients received 20 minutes of physical therapy along with 20 minutes of biofeedback training three times a week for five weeks. The third group of three subjects received only functional electrical stimulation (FES) for the five week duration. Gait patterns using a stroboscopic cinematography were recorded at the initial visit and after the completion of the training program. The results indicated that the physical therapy group showed slight improvement in gait patterns but were not considered remarkable. Two of the three subjects in the FES group showed improvement in gait patterns even when not using the stimulator device. The biofeedback group showed more substantial improvements in the overall gait pattern.

In a separate clinical study, 16 patients with abnormal gait patterns as a result of drop-foot were evaluated (Intiso et al. 1994). The subjects were randomly placed into either a control group or EMG-BFB group. The 8 patients within the control group received only 60 minutes of daily physical therapy based on the Bobath method for 2 months. The EMG-BFB group received a combination of physical therapy and biofeedback training for the same interval of time. Evaluations of the subject's initial and final capabilities were done using various clinical neurological examinations and functional scales. In addition, a kinesiological assessment was also performed using motion capture applications. The results showed a significant difference between initial and final scores on two of the five neurological and functional exams for the EMG-BFB group. No statistically significant differences were found in the control group. The 
kinematic analysis results from the motion analysis showed significant recovery in the ankle dorsiflexion during swing phase from $130.9^{\circ}$ to $119.1^{\circ}(\mathrm{P}<0.02)$ in the EMG-BFB group. No statistically significant results were obtained from the control group. In a separate 1 year follow-up study of 54 stroke patients who received only biofeedback training, the results showed that 39 of the 54 patients had retained their functional recovery skills at the time of the reassessment (Wolf 1980). This indicates that biofeedback training does provide lasting neurological improvements. 


\section{STATEMENT OF PURPOSE}

The pathology of stoke primarily attacks the central nervous system which includes the cerebrum. With the cerebrum serving as the primary center for higher brain functions, memory, and the regulation of skeletal muscles contraction (Martini et al. 2003), damage to this portion of the CNS will commonly result in various forms of motor function disorders. Paralysis or paresis of the dorsiflexion muscle results in a condition known as drop-foot where the user is unable to voluntarily raise their ankle during the swing phase. This alters the gait pattern in a way that handicaps the user's ability to walk.

The most common treatment for drop-foot includes the use of a thermoplastic ankle-foot orthosis. The semi-rigid brace passively holds the users foot in a neutral position during the swing phase correcting the drop-foot condition. The solution corrects the superficial problem, but does not attempt to improve the underlying cause of the handicap which is the loss of voluntary muscular control. New rehabilitation techniques have been created focused on retraining the user's mental capability to revitalize their capacity to regain neuromuscular control. Treadmill training improves gait pattern by allowing the patient to perform gait specific exercises in a repetitive motion. Robotic assistive devices programmed to assist the user in completing active movement exercises have shown dramatic improvement in voluntary muscular control. And the use of biofeedback in active exercise programs resulted in improved gait and muscular coordination. Each of these rehabilitation techniques provides individually unique and 
beneficial characteristics which have shown to help improve the level of recovery in stroke patients.

The focus of this research thesis is to develop a tool that can be used as a rehabilitation device which incorporates the best-of characteristics from other forms of rehabilitation techniques into a single integrated package. The Biofeedback Assistive Robotic Ankle-foot orthosis (B.A.R.A.) is intended to provide an instrument to be used by stroke patients in a home or rehabilitation environment that offers neuromuscular and gait training exercises. This requires the development of a new device that is low-cost, portable, durable, and easy to use tool. The specific aims of this device are as follows,

- Specific Aim \#1: Develop a stationary assistive repetitive motion training tool focused on improving the user's capability to voluntarily dorsiflex their ankle by promoting muscular contraction of the tibialis anterior muscle through EMG biofeedback

Test Hypothesis \#1: Biofeedback training in conjunction with assistive movement of the impaired ankle improves neuromuscular control in stroke patients

- Specific Aim \#2: Develop a gait exercise training device designed to provide a more symmetrical gait pattern of the ankle by correcting the conditions of swing phase drop-foot through EMG biofeedback

Test Hypothesis \#2: The use of a biofeedback controlled assistive AFO will improve gait symmetry in stoke patients

The premise for the creation of the BARA is not intended to be a permanent training tool to be used by the stroke patient in an on-going exercise regiment. Rather, the end goal is for the rehabilitation device to provide substantial neuromuscular reeducation to allow 
the patient to become independent on the use of walking aids and regain their natural walking capabilities. 


\section{DESIGN PARAMETERS}

The BARA device will integrate the use of the patient's own electromyography signal to provide an external sensory feedback to supplement the natural proprioceptive awareness which may have been lost due to the complications of stroke. The BARA will also have the capability to assist the user in completing their physical movements through mechanical actuation of the ankle utilizing a robotic algorithmic control system. The device must also allow the user to participate in walking exercises that will not altering the typical gait patterns, in addition will includes a safety system to help prevent the effects of drop-foot during the swing phase.

Based on the intended use of the BARA device, several requirements were first established which had to be satisfied when creating the overall design of the BARA. The requirements are,

- The device must be portable to be easily transported between locations

- The device must allow the user the ability to sit comfortably in a standard chair with the device on

- The device should provide the user the ability to don and doff the equipment easily

- The device must function in a typical environment without additional specialized equipments and outputs

- The maximum weight should be less than $10 \mathrm{lbs}$.

- The device must include safety measures to prevent the foot from dragging during the swing phase of gait 
- The device should allow general size fitting to accommodate multiple users

- The device must be non-invasive

- The device should require minimal maintenance to operate

- The device should have a simple user interface requiring minimal direction to operate

- The device should allow the user at least an 8 meter walking range

- The device must be comfortable to the user

Based on the requirements listed above, the following BARA design was created and implemented for this research thesis.

\subsection{Ankle-Foot Orthosis}

In order to maintain the overall design parameters of the BARA device, the anklefoot orthosis device had to adhere to the following three requirements,

- Does not impede the natural walking motion

- Provides enough surface area and rigidity to attach necessary components

- General size and fitting to allow various users to wear the device

A decision was made to use a commercially available walking brace to reduce the cost and work associated with building a custom frame. The Pro-Rom Walker (Model \#: 7995045, Size M) was selected as a suitable device which met all the necessary requirements (Figure 4.1). The Pro-Rom Walker is originally designed to help treat patients with acute ankle sprains, fractures of the lower leg and ankle, and following achilles tendon surgery. 


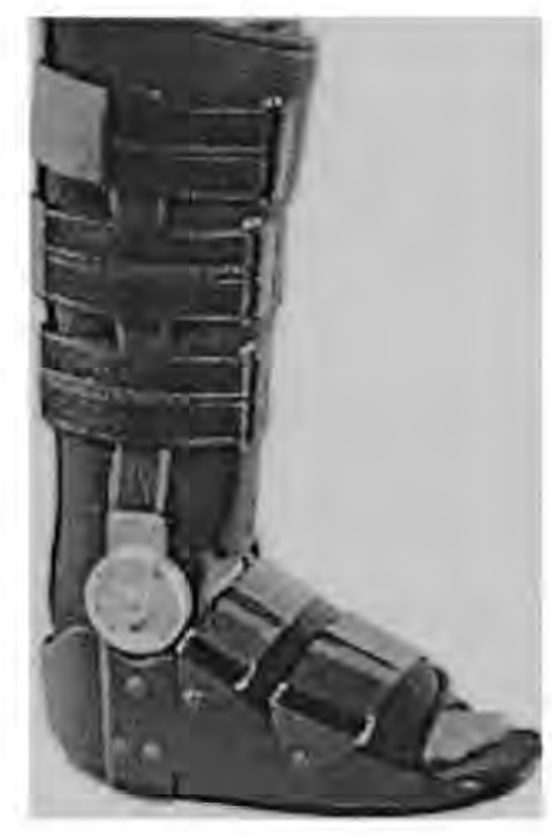

Figure 4.1 Pro-Rom Walker

The device is specifically designed to be used as a walking cast and includes an ankle hinge with allows for free motion about the ankle. The device allows for a maximum ROM of $45^{\circ}$ plantar flexion and $30^{\circ}$ dorsiflexion, similar to that of a normal ankle. Predrilled holes within the ankle hinge provide a protected range of motion in $7.5^{\circ}$ increments for both plantar flexion and dorsiflexion. Because the device is meant to be used for gait rehabilitation, where the joint angle of the ankle-foot complex ranges around $20^{\circ}$ plantar flexion and $10^{\circ}$ dorsiflexion for a typical gait pattern (Perry 1992), the restriction pins were set so as to only allow a maximum of $37.5^{\circ}$ plantar flexion and $22.5^{\circ}$ dorsiflexion as a safety precaution for the intended user.

The leg support is made out of aluminum bars which provide a very rigid support structure onto which additional components can be attached to the AFO. The foot-base is constructed out of plastic with a low profile rocker bottom design with a non-skid sole 
which assists in a smooth forward rocking motion during stance period. A foam insert is included in the base to provide shock absorption and comfort. A breathable sock-like liner is worn over the leg of the user to provide a form fit and assists in securing the AFO to the user's leg using velcro straps.

Additional components had to be custom fabricated and added to the AFO to provide anchor points onto which to secure necessary mechanical and electrical devices. The Five custom components that were manufactured for use with the AFO brace are:

a) Calf connector

b) Calf connector hinge

c) Heel connector hinge

d) Encoder cap

e) Encoder mount

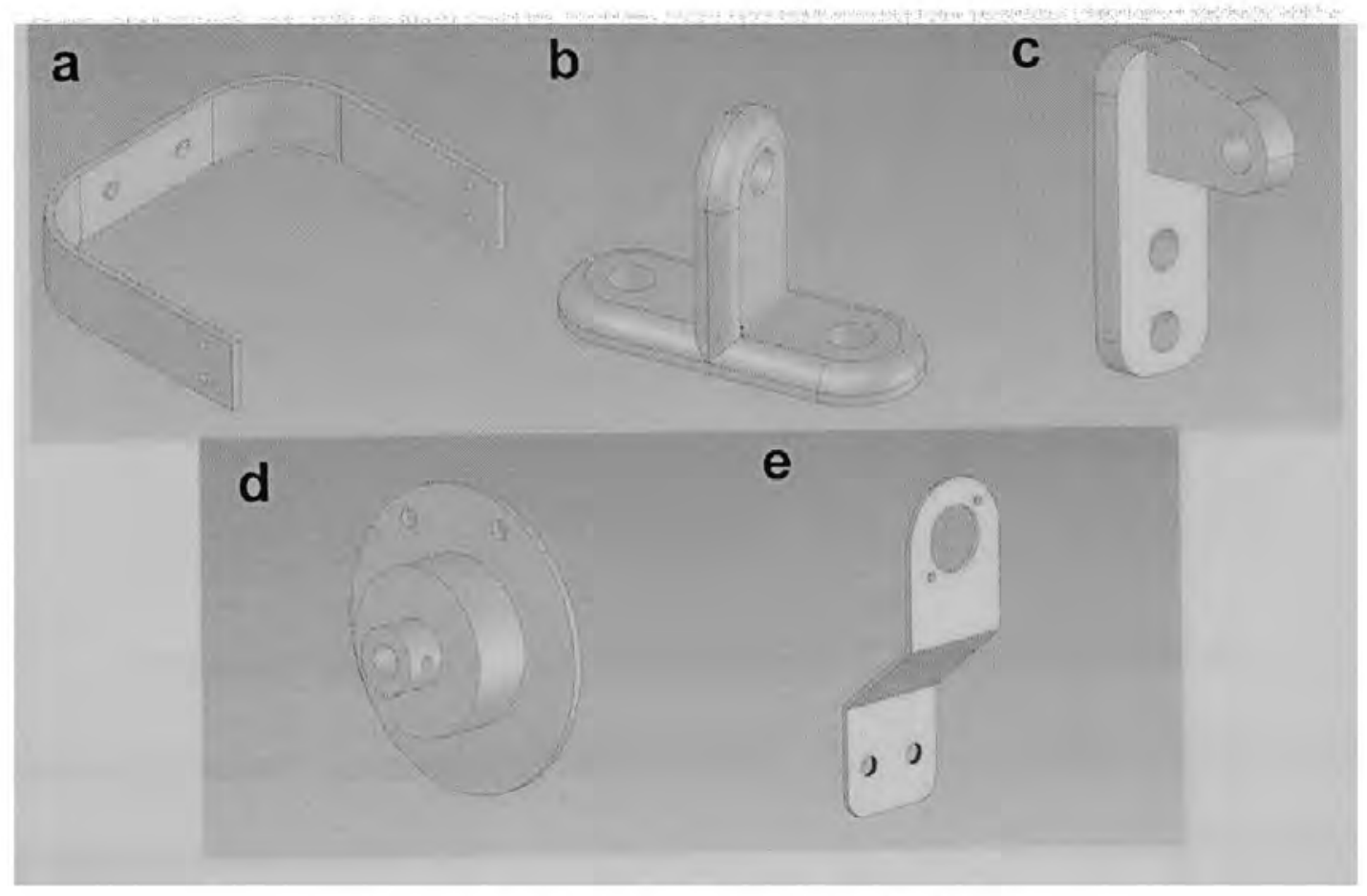

Figure 4.2 Custom components for AFO brace 
Figure 4.2 provides a 3 -dimensional rendering of the five individual components (See Appendix A for engineering drawing with dimensions). The components are assembled to the AFO by hand. The calf connector and calf connector hinge is secured to the aluminum support bars on the AFO and provides a point of attachment for connecting the actuator frame to the AFO. The heel connector hinge is attached to the rear of the footbase and provides the anchor point for connecting the actuator piston to the AFO.

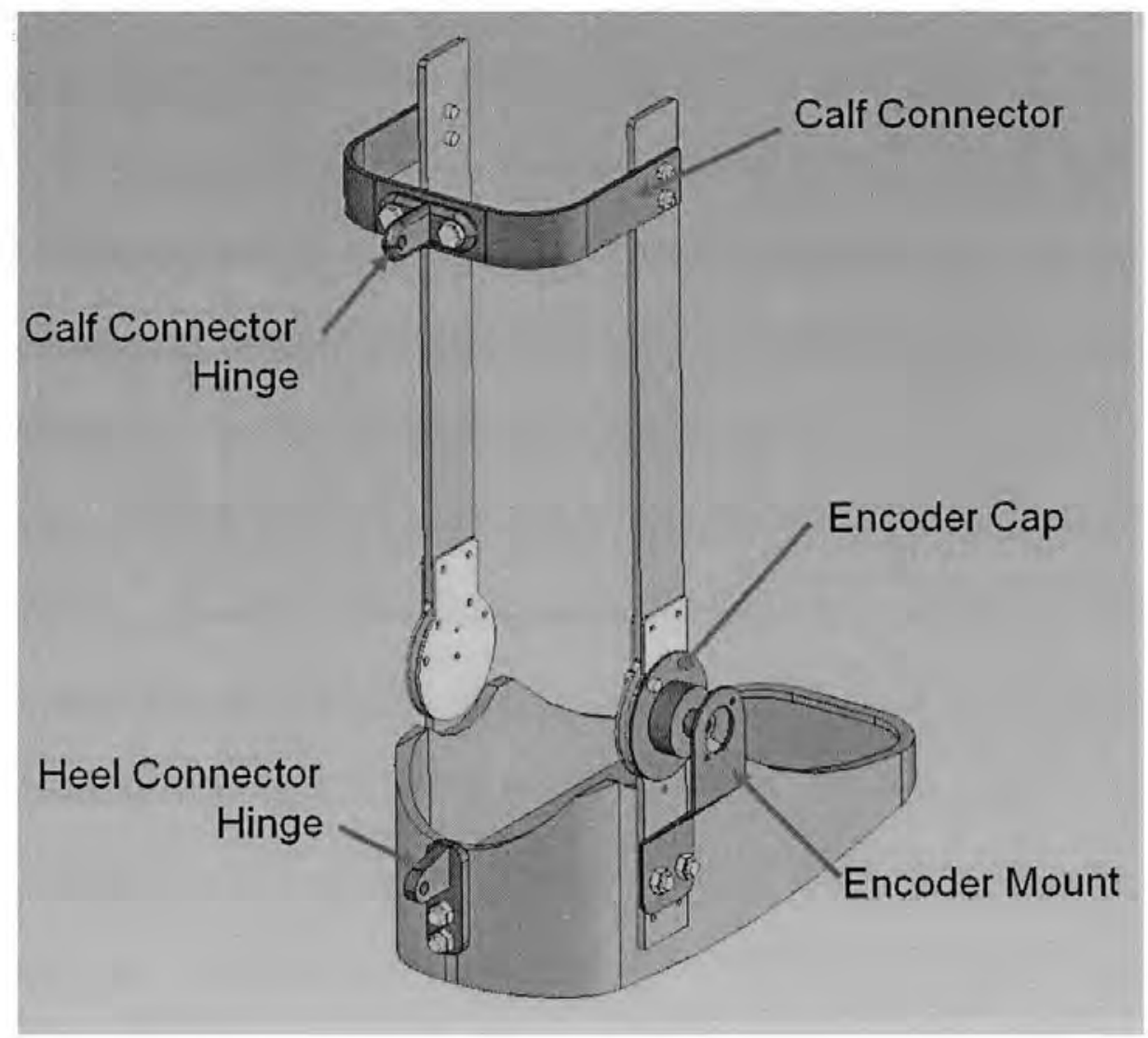

Figure 4.3 Assembled Ankle-Foot Orthosis with custom components attached

The encoder mount is connected directly to the foot-base and aligned so that the shaft of the encoder will be in-line with the ankle hinge. The encoder cap is secured to the outer right ankle hinge. The central axis is directly in-line with that of the ankle hinge and the 
design includes a set screw collar for securing the encoder shaft in position.. Figure 4.3 shows an illustration of the AFO with the custom components assembled.

\subsection{Actuator}

\subsubsection{Ball Screw}

A ball screw was incorporated into the design because it provides a mechanical device that translates rotary motion into linear motion and vice versa. They are capable of providing high precision movement in addition to being able to apply or withstand high thrust loads. The ball screw is composed of two main components, (1) a spiral shaft, and (2) a specialized ball assembly nut. The frictional contact between the spiral shaft and the ball assembly is applied through the contact of the ball bearings situated between both components. The balls are allowed to freely roll within the ball assembly which is re-circulated. The decrease in contact surface area dramatically reduces the amount of internal friction. This allows balls screws to operate at approximately $90 \%$ efficiency.

A semi-custom built ball screw manufactured by Misumi USA Inc. (Part No. BSSZ1004-219-RLC) was used within the BARA device. The ball screw shaft consisted of a thread diameter of $10 \mathrm{~mm}$ and a lead length of $4 \mathrm{~mm}(\mathrm{Dia}=0.393 \mathrm{in} ., \mathrm{Lead}=0.157$ in.) (Figure 4.4). The spiral shaft of the ball screw assembly was further modified by reducing the length of the fixed side end to 0.5 " (see Appendix A for engineering drawing and dimensions).

The threaded area on the spiral shaft equates to the maximum length of travel the ball bearing assembly can displace linearly which corresponds to the maximum amount of angular movement that can be applied to the ankle. The travel length of the ball screw 
required for the BARA device was finalized by generating a detailed 3-dimensional model of the entire BARA using the Solidworks CAD program. Using the set design parameters for the arc of motion of the ankle not to exceed $60^{\circ}\left(37.5^{\circ}\right.$ plantar flexion + $22.5^{\circ}$ dorsiflexion $=60^{\circ}$ ), it was determined that a threaded length of 6.5 inches would meet both the functional and design requirements.

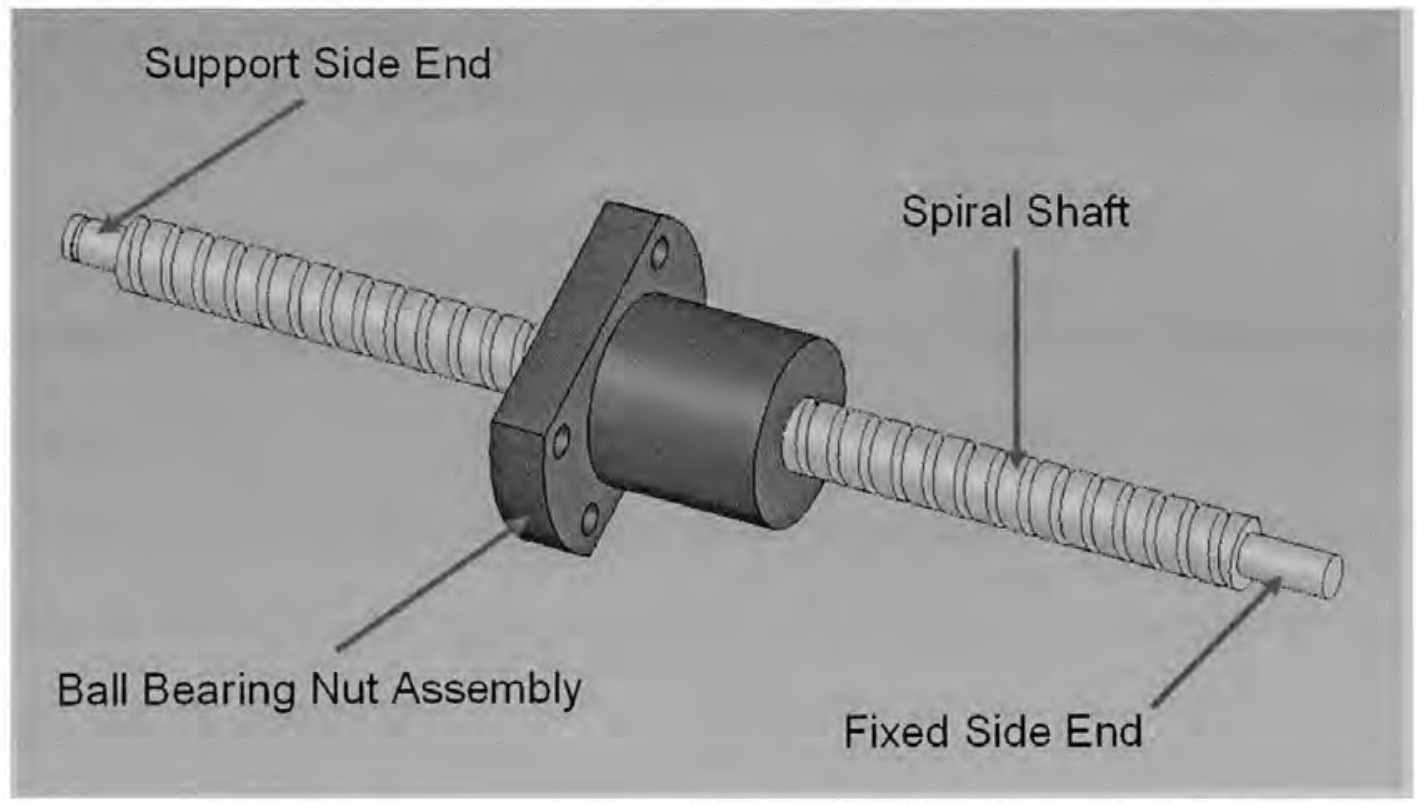

Figure 4.4 Semi-custom built ball screw assembly

Two equations were used to help calculate important design factors governing the use of ball screws (Danaher Motion, 2006). The first equation determines the rotational speed of the ball screw shaft required to achieve a specific linear velocity (Eq. 4.1).

$$
n(R P M)=\frac{\text { Travel_Rate }\left(\text { in } x \text { min } .^{-1}\right)}{\text { Lead_Length }(\text { in })}
$$

Based on the design of the BARA brace a Travel rate of $4 \mathrm{in} / \mathrm{sec}=240 \mathrm{in} / \mathrm{min}$ was deemed to be an acceptable velocity to reposition the foot into a neutral position during swing phase. The calculated rotational speed (n) is equal to, 


$$
n=\frac{240 \frac{\mathrm{in}}{\mathrm{min}}}{0.157 \mathrm{in}}=1529 R P M
$$

The second equation calculates the necessary driving torque $\left(T_{d}\right)$ required to achieve linear motion (Eq. 4.2).

$$
\begin{aligned}
& T_{d}\left(l b_{f}-\text { in.. }\right)=\frac{F P e}{2 \pi}=0.177 F P \\
& F={\text { Equivalent_Operating_Load }\left(l b_{f}\right)}_{P}=\text { Lead }(\text { in }) \\
& e=\text { Efficiency }=0.90
\end{aligned}
$$

An efficiency rating of $90 \%$ was used based on typical ball screw design parameter (Danaher Motion 2004). Considering the nature of the target patient who exhibits a flaccid muscle tone, an operating load of $40 \mathrm{lb}_{\mathrm{f}}$ was considered more than an adequate amount of force generated within a patient's ankle. Therefore the maximum driving torque was calculated to be,

$$
T_{d}=0.177 * 40 l b_{f} * 0.157 \text { in }=1.112\left(l b_{f}-\text { in }\right)
$$

\subsubsection{Support Frame}

The function of the actuator support frame is designed to, (1) Provide a rigid framework to securely anchor the DC motor and the support side of the ball screw shaft, (2) Present a central axis of alignment between the shaft of the DC motor, the clutch, the ball screw, and the piston frame, (3) Include an attachment site that allows the actuator component to be easily attached/removed from the AFO. 
The support frame is composed of 4 separate custom manufactured pieces which are assembled together by hand using bolts and nuts to form the complete frame. The custom pieces are:

1. Actuator frame hinge

2. Top plate

3. Base plate

4. Support columns

Figure 4.5 illustrates the assembled support frame in its entirety (see Appendix A for engineering drawing and dimensions).

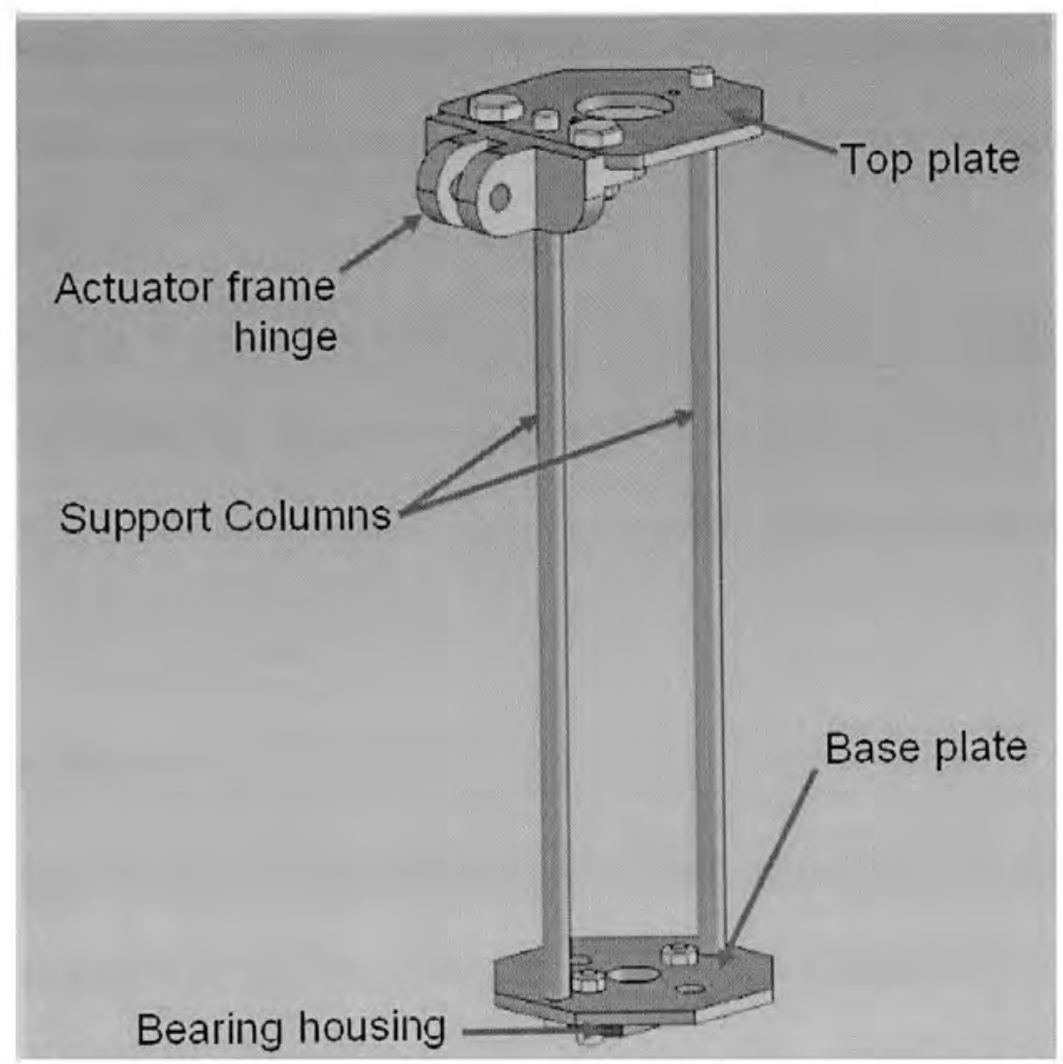

Figure 4.5 Assembled actuator support frame 
The actuator frame hinge is made out of aluminum and designed with two parallel ridges that align with the calf connector hinge to provide a point of attaching the actuator to the AFO using a quick release pin. The remaining custom components are fabricated from steel to provide strength. The top plate has several predrilled holes used to allow the connecting bolts for the support column, frame hinge, and motor to pass through. The next component is a pair of supporting columns made of steel rods with holes drilled and threaded on either end. The connecting bolts screw directly into the ends of the steel rods. The base plate has similar predrilled holes to allow the connecting bolts for the support column and bearing holder. In addition there is a pair of guide holes used by the actuator piston frame to keep the motion inline with the axis of travel. It is essential that the predrilled holes used to attach the motor, support columns, and bearing are all inline to allow parallel axes.

The bearing housing is a commercial piece purchased from Misumi USA Inc. (Part No. SBACN606ZZ). It consists of an aluminum housing which encases a collar bearing used to hold the support side of the ball bearing in place and allows the shaft to rotate freely.

\subsubsection{Piston Frame}

The piston frame is also a custom manufactured component. Its primary role is to serve as a fixed length bridge that connects the ball bearing assembly of the ball screw to the heel of the AFO. Figure 4.6 shows the assembled piston frame which is constructed from two custom manufactured pieces. The first component is the pair of steel support columns. They are made out of steel rods with threaded holes on either end. The support 
columns connect to the piston head which is an aluminum piece with two parallel ridges which align with the hell connector hinge to provide the second point of attachment for connecting the actuator to the AFO.

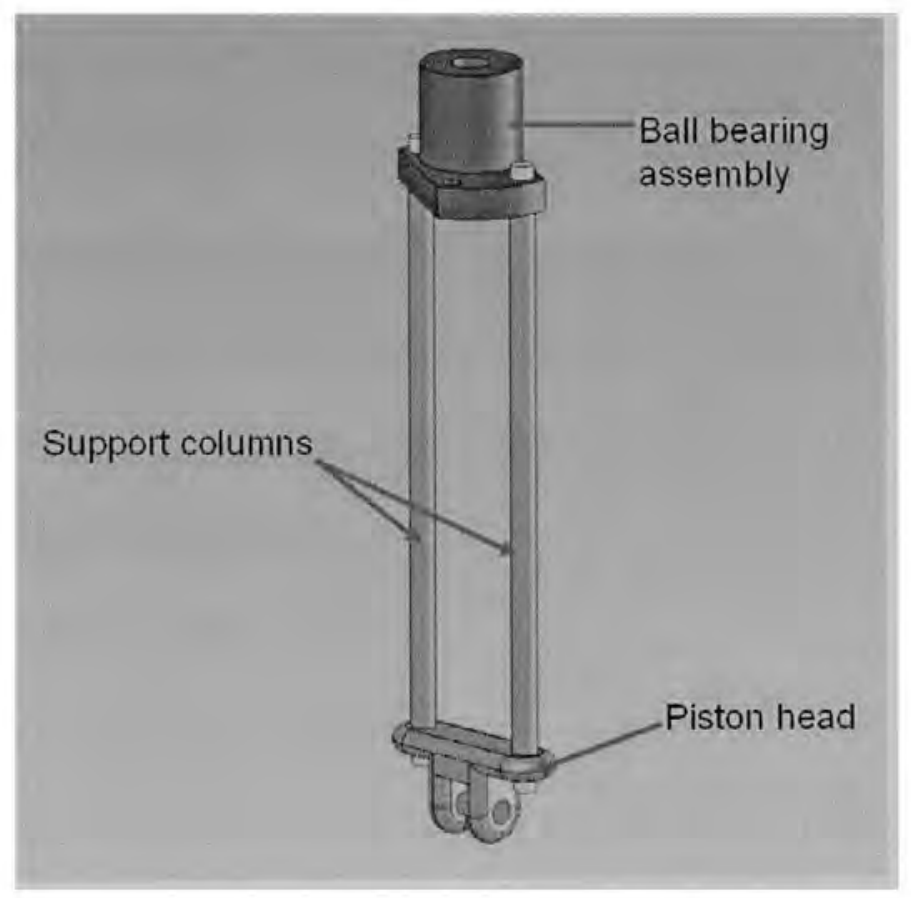

Figure 4.6 Assembled piston frame

The piston frame acts as an extension of the ball bearing assembly and directly transfers the linear motion into a push/pull force on the heel of the AFO. This in turn will result in the angular movement around the ankle due to the moment force created by the piston frame. The support frames run through the guide holes located on the base plate of the support frame. This insures the movement of the piston frame is inline with the axis of the ball screw. 


\subsubsection{Brush Motor}

The DC motor provides the mechanical energy for which to power the movement of the ball screw. A brushed DC Motor was selected for use with the BARA because of their general availability, lower cost, and simpler control interface requirements. The selection of the motor was based on the following design and performance requirements,

1. A nominal performance speed of at least 1529 RPM to satisfy the desired 4 $\mathrm{in} / \mathrm{sec}$ linear velocity of using the selected ball screw

2. A maximum torque output of at least $1.112 \mathrm{lb}_{\mathrm{f}}$-in $=125.64 \mathrm{mN}-\mathrm{m}$ based on the calculation result for use with the selected ball screw

3. Overall length of the less than 5 "

4. Maximum diameter less than 3"

5. Operating voltage of $12 \mathrm{~V}$ or less

The Couzet DC motor Model \#82-800-036 was chosen because it satisfied all the performance and design requirements. Figure 4.7 shows the schematic dimension of the Crouzet motor. Table 4.1 provides the performance specifications of the motor.
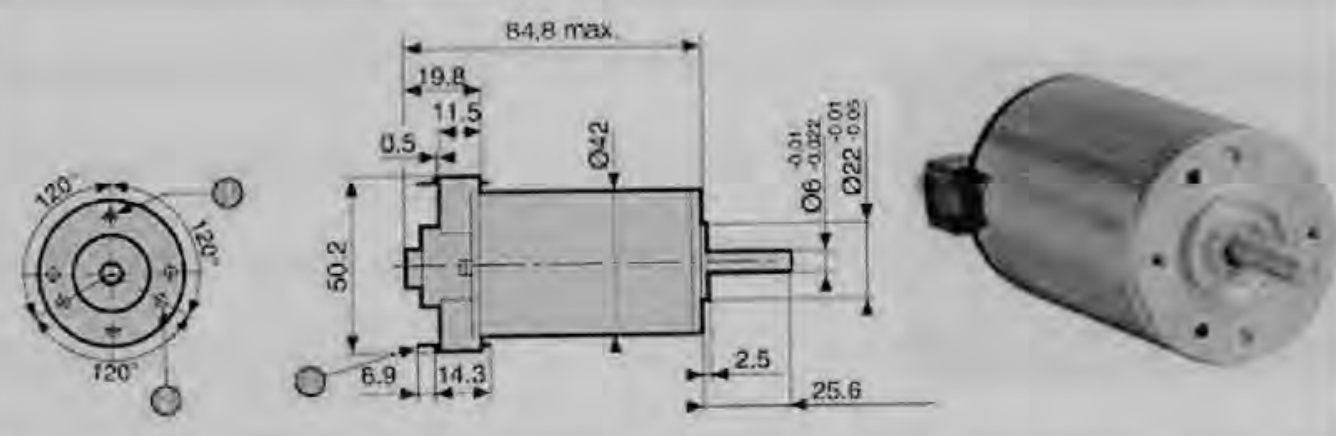

Figure 4. 7 Crouzet DC brushed motor dimension 


\begin{tabular}{|l|c|c|}
\hline \multicolumn{1}{|c|}{ Characteristics } & Units & Value \\
\hline Nominal Voltage & $\mathrm{V}$ & 12 \\
\hline Nominal Speed & $\mathrm{rpm}$ & 2000 \\
\hline Starting Torque & $\mathrm{mN}-\mathrm{m}$ & 185 \\
\hline Diameter & in & 1.65 \\
\hline Overall Length & in & 4.35 \\
\hline Weight & $\mathrm{g}$ & 400 \\
\hline
\end{tabular}

Table 4.1 Crouzet motor specifications

\subsubsection{Electromagnetic Clutch}

An electromagnetic clutch is a mechanical device used to transmit rotational movement. The device allows the coupling between the motor and load to be engaged/disengaged through the means of an electromagnet. The device is comprised of two main assemblies: (1) rotor assembly, (2) hub assembly. The rotor assembly contains a magnetic coil which magnetizes when a current is passed through. The magnetic flux generated pulls the hub assembly toward the rotor assembly and a secure contact is formed through two high friction surfaces. The transfer of torque is achieved through mechanical friction.

An electromagnetic clutch was integrated into the design after the initial direct coupling between the motor and ball screw exhibited higher than desired rotational impedance. The torsional impedance inherent in turning the DC motor's shaft "by hand" made it difficult for the linear thrust on the ball bearing assembly to translate into rotational movement of the ball screw and motor shaft that was generated from the free motion of the user's action. The type SO-11 electromagnetic clutch by Inertia Dynamics (Model No.: M0710-3311) was incorporated as the coupling system between the motor shaft and 
ball screw. Figure 4.8 provides an illustration of the clutch assembly along with the schematic dimension.

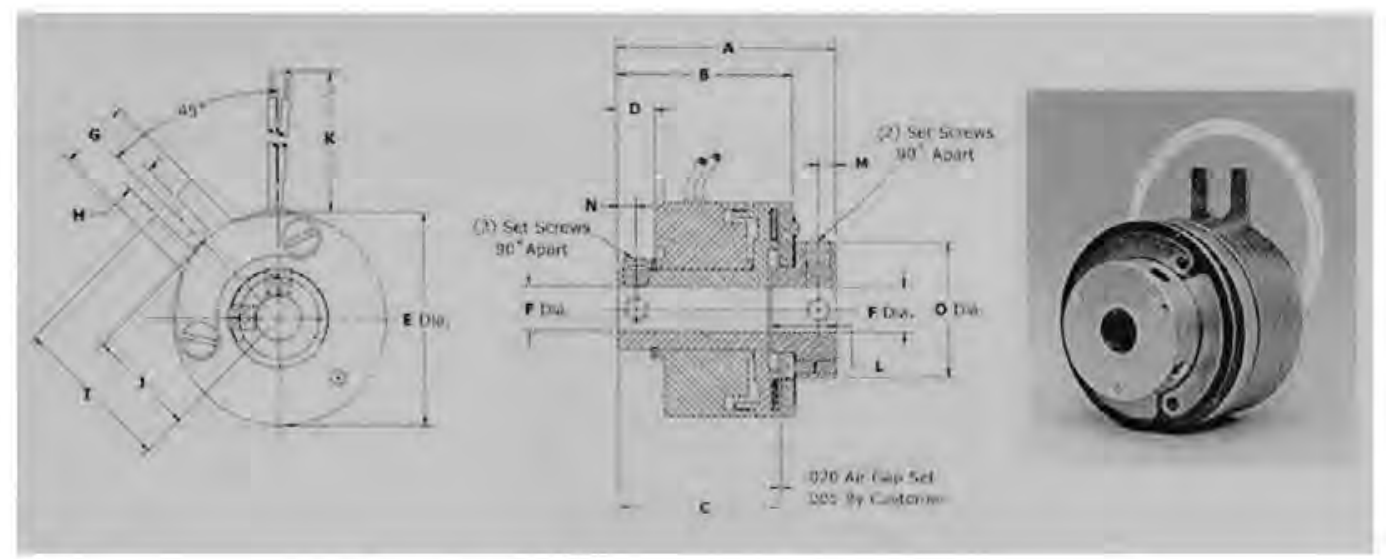

\begin{tabular}{|c|c|c|c|}
\hline & $\begin{array}{c}\text { Dimension } \\
\text { (in.) }\end{array}$ & $\begin{array}{c}\text { Dimension } \\
\text { (in.) }\end{array}$ \\
\hline A & 1.168 & I & 0.875 \\
\hline B & 0.933 & J & 0.585 \\
\hline C & 0.777 & K & 12.00 \\
\hline D & 0.147 & L & 0.307 \\
\hline E & 1.160 & M & 0.093 \\
\hline F & $5 / 16$ & N & 0.087 \\
\hline G & 0.380 & O & 0.687 \\
\hline H & 0.122 & & \\
\hline
\end{tabular}

Figure 4.8 SO-11 Electromagnetic clutch

Table 4.2 provides the specification of the device.

\begin{tabular}{|l|c|c|}
\hline \multicolumn{1}{|c|}{ Description } & Unit & Value \\
\hline Weight & $\mathrm{oz}$ & 3.2 \\
\hline Static Torque & $\mathrm{lb}-\mathrm{in}$ & 6 \\
\hline Voltage & $\mathrm{V}$ & 12 \\
\hline Current & $\mathrm{Amp}$ & 0.447 \\
\hline
\end{tabular}

Table 4.2 SO-11 electromagnetic clutch cpecifications

When the electromagnetic clutch is disengaged, the rotor and hub assembly are separated by a $0.005 "-0.020$ " air gap between the two components. This essentially eliminates the torsional impedance effect of the motor shaft on the ball screw while still maintaining a 
central alignment of the two axes. This allows the users to freely actuate their ankle with minimal resistance while the clutch is disengaged.

Because both the motor and electromagnetic clutch are off-the-shelf items, there was a difference in diameter between the motor shaft and the collar of the clutch.

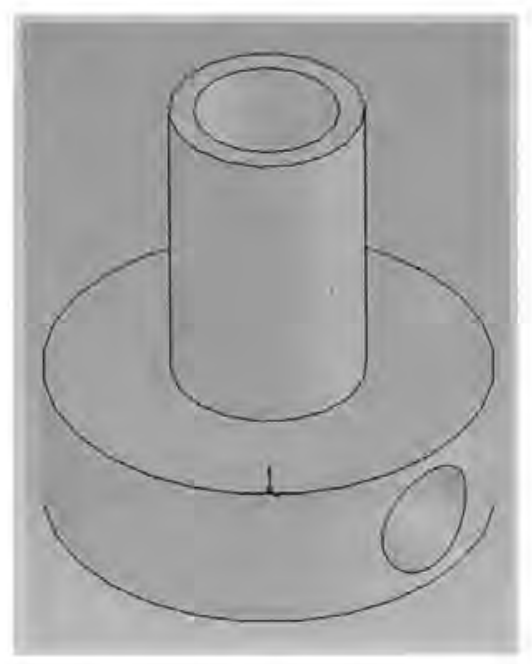

Figure 4.9 Clutch sleeve

In order to maintain proper alignment of the two axes, a custom aluminum clutch sleeve was manufactured to provide a proper fitting (Figure 4.9) (See Appendix A for engineering drawing with dimensions). The clutch sleeve is assembled between the motor shaft and the rotor assembly. Figure 4.10 provides an exploded view of the entire assembly. The through hole in the clutch sleeve allows the motor shaft to properly fit within the diameter of the sleeve. It is secured using two set screws aligned $180^{\circ}$ apart. The outer diameter of the lower column of the sleeve matches the inner diameter of the rotor assembly collar allowing a tight fit. This is also secures using the existing set screws. 


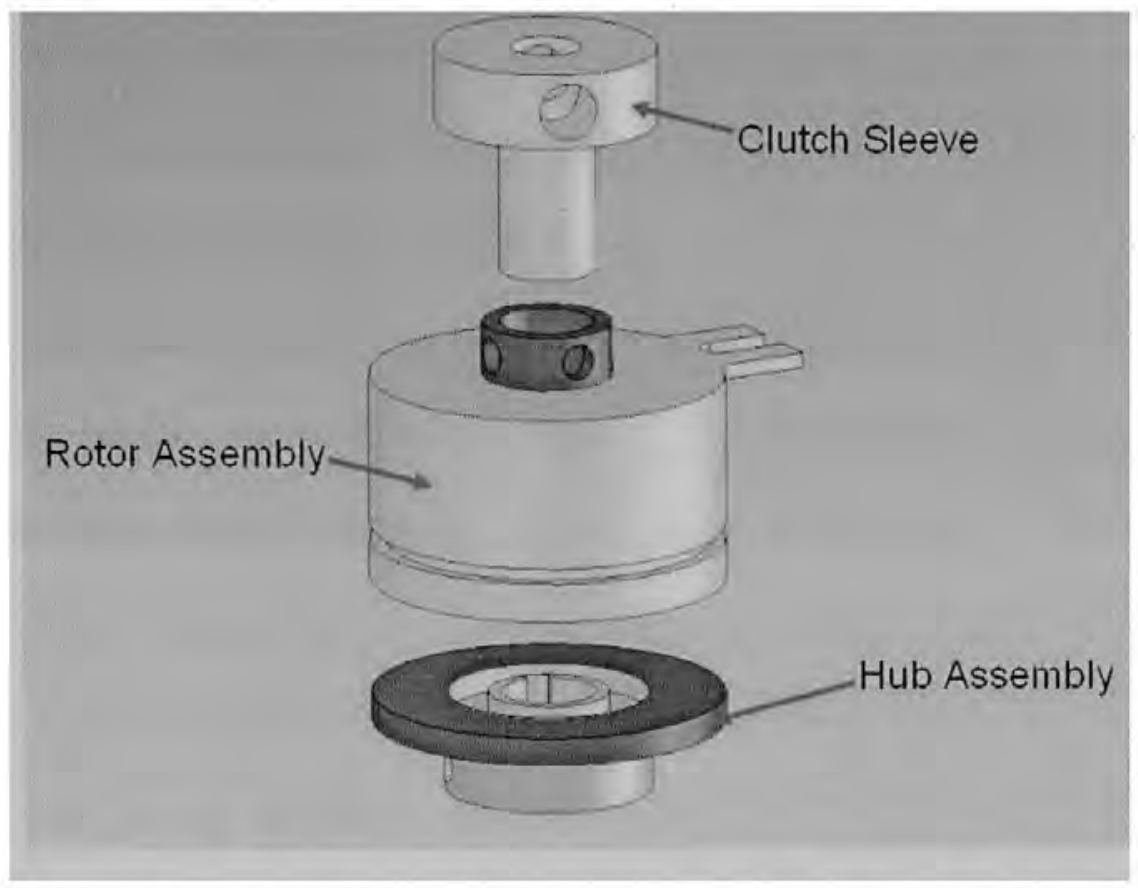

Figure 4.10 Exploded view of clutch assembly components

\subsection{EMG Device}

The role of the EMG device serves to provide the vital biofeedback input into the entire gait rehabilitation process. It accomplishes two tasks which are (1) the surface electrode are able to monitor the intensity of voluntary muscle contractions which requires the active participation of the user, and (2) the device provides some forms of substitute sensory feedback (e.g. visual or audio) to the user to help compensate for lost proprioceptive senses. The requirements for the biofeedback device includes,

1. Design must include a minimum of 1-channel input capable of monitoring the myoelectric signal of the user without being invasive

2. Device must be adjustable to customize to the specific need of the user and provide incremental increases in setting to follow improvement strategies 
3. Must provide some form of alternative sensory feedback to the user

4. Device must include an output capable of serving as an initiation trigger that can be connected to an external equipment

5. The unit must be portable to allow use with ambulatory exercises The MyoTrac (Model No. SA4000P) by Thought Technologies Ltd. was selected as the EMG Biofeedback device to be used with the BARA (Figure 4.11). The device uses disposable surface triodes (Model \# T3402) using a sensor cable (Model \# T9385M).

The device features instrumental controls for feedback setting (i.e. continuous or threshold), gain setting, threshold adjustment setting, a 13 LED light bar display, and audio volume control. The device also includes input connectors on its side for connecting the sensor cable, ear phones, and a $2.5 \mathrm{~mm}$ jack for the $0-2 \mathrm{~V}$ output. Table 4.3 outlines the specifications of the device.

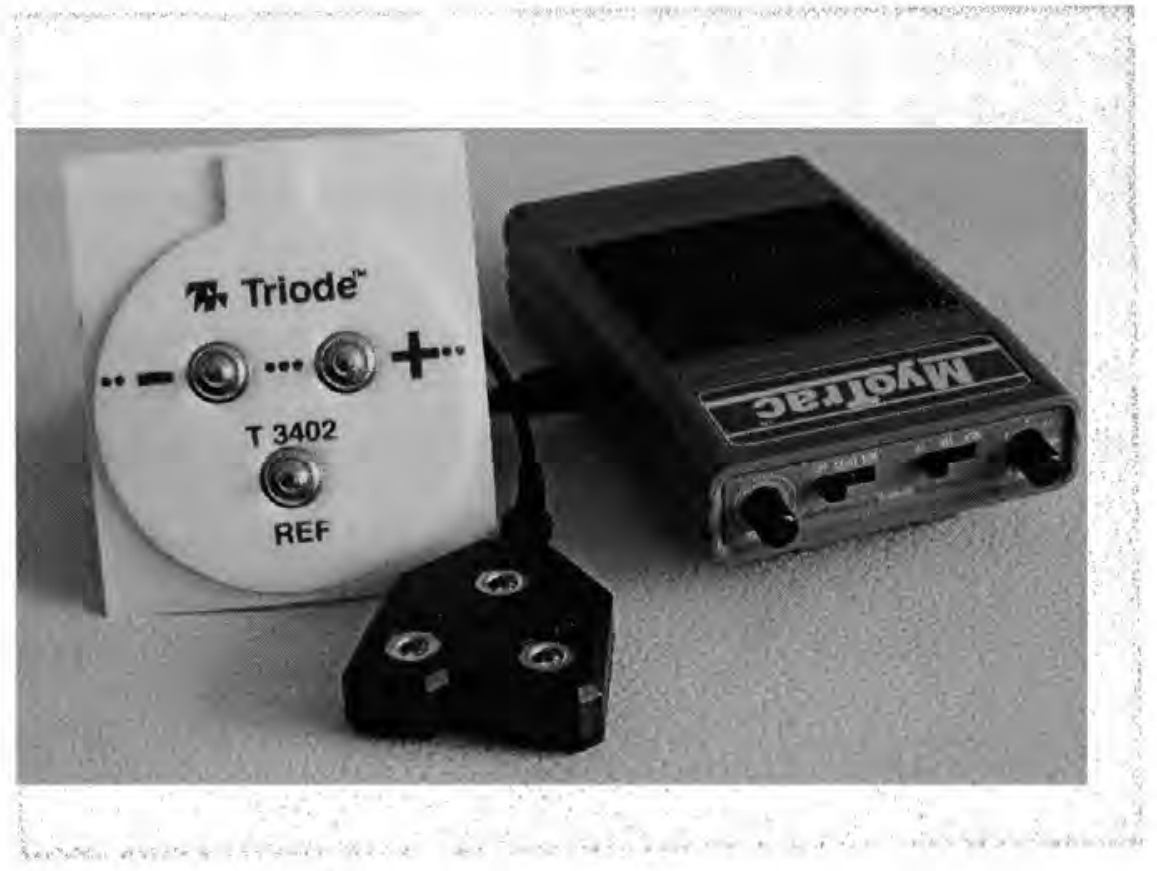

Figure 4.11 Myotrac EMG device with sensor cable and Triode 


\begin{tabular}{|l|l|}
\hline \multicolumn{2}{|l|}{ MyoTrac SA4000P Specifications } \\
\hline Size & $2.4 " \times 4.4 " x ~ 1 "$ \\
\hline Weight & $2.5 \mathrm{oz}$ \\
\hline Gain Settings & $\mathrm{x} 1, \mathrm{x} 10, \mathrm{x} 100$ \\
\hline Signal Input Range $\mathrm{x} 1$ & $0-20 \mu \mathrm{V}$ \\
\hline Signal Input Range $\mathrm{x} 10$ & $0-200 \mu \mathrm{V}$ \\
\hline Signal Input Range $\mathrm{x} 100$ & $0-2000 \mu \mathrm{V}$ \\
\hline Threshold Control & $0.5-10$ \\
\hline Signal Output Range & $0 \mathrm{~V}-2 \mathrm{~V}$ \\
\hline
\end{tabular}

Table 4.3 MyoTrac Specifications

The threshold feedback setting was utilized because it provides control settings to adjust the proportional audio tone and visual light bar to enable when the EMG signal exceeds the desired threshold control value. This provides the substitute sensory biofeedback to the user to indicate their level of activity and also allows the settings to be personalized for specific users.

The $0-2 \mathrm{~V}$ output from the MyoTrac was used as the trigger signal for initiating user defined motion on the BARA. A minimum output of $1.5 \mathrm{~V}$ was necessary to initiate the movement sequence on the control system. This required an EMG output reading of at least $15 \mu \mathrm{V}, 150 \mu \mathrm{V}$, or $1500 \mu \mathrm{V}$ depending on the gain setting (i.e. $\mathrm{x} 1$, $\mathrm{x} 10$, or $\mathrm{x} 100$ ).

\subsection{Foot Switches}

Foot switches are necessary to help determine whether the target foot is in the stance or swing period of the gait cycle. When the foot switches are active, this signifies that some portion of the body weight is being carried by the foot representing the stance period when the foot is in contact with the ground. When all the switches are inactive, this represents the swing phase characterized by the lifting of the foot off the ground resulting in a no-load situation. Two foot switches were used with the design of the 
BARA. The placement of the two foot switches was located at the heel and ball of the right foot to capture the initiation and termination of the stance phase (i.e. initial contact and pre-swing).

The foot switch was created using a semi-custom Flexiforce load sensors (Model No. 1207) by manufactured by Tekscan. The load sensor is an ultra-thin and flexible printed circuit with an active sensing area of 1" in diameter. The sensor uses a resistivebased technology which provides a change in the resistance of the sensor element inverse to the applied load. Table 4.4 provides some of the specification of the Flexiforce sensor. Applications for this type of sensor include identifying force thresholds to trigger specific actions. The force threshold will be adjustable to provide a signal to the control system when an acceptable weight is applied to the load sensors signifying the initiation or termination of stance.

\begin{tabular}{|l|c|}
\hline Flexiforce Model No. 1207 & $0.014 "$ \\
\hline Thickness & $1.80 "$ \\
\hline Length & $1.25 "$ \\
\hline Diameter of sensor & $1 "$ \\
\hline Sensing Area (Diameter) & 2-pin male square pin \\
\hline Connector & $>40 \mathrm{M} \Omega$ \\
\hline Resistance at no-load & $\sim 40 \mathrm{~K} \Omega$ \\
\hline Resistance at 165 lbs & \\
\hline
\end{tabular}

Table 4.4 Flexiforce load sensor model 1207 specification

The foot switch assembly is constructed using the Flexiforce sensor, a 1.25" diameter aluminum disk, and a 1" diameter plastic disk. Double sided adhesive tape is placed one either side of the aluminum and plastic disk.

The aluminum disk is attached to the underside of the Flexiforce sensor using the adhesive tape. The plastic disk is attached to the top side of the Flexiforce sensor 
centered in the middle of the sensor area. Figure 4.12 shows the individual components of the foot switch along with the assembled component. The plastic disk helps to evenly distribute the load onto the sensor area and the aluminum disk provides a rigid backing for the sensor to rest upon. A Waldom 2-pin connector and crimps (Part No. 22-01-3027 \& 08-50-0114) were used to connect the 2-pin terminal of the foot switch to the 20-gauge wire used to connect to the control system. Electrical tape was carefully used to cover the entire foot switch and secure the connector to protect it from outside elements.

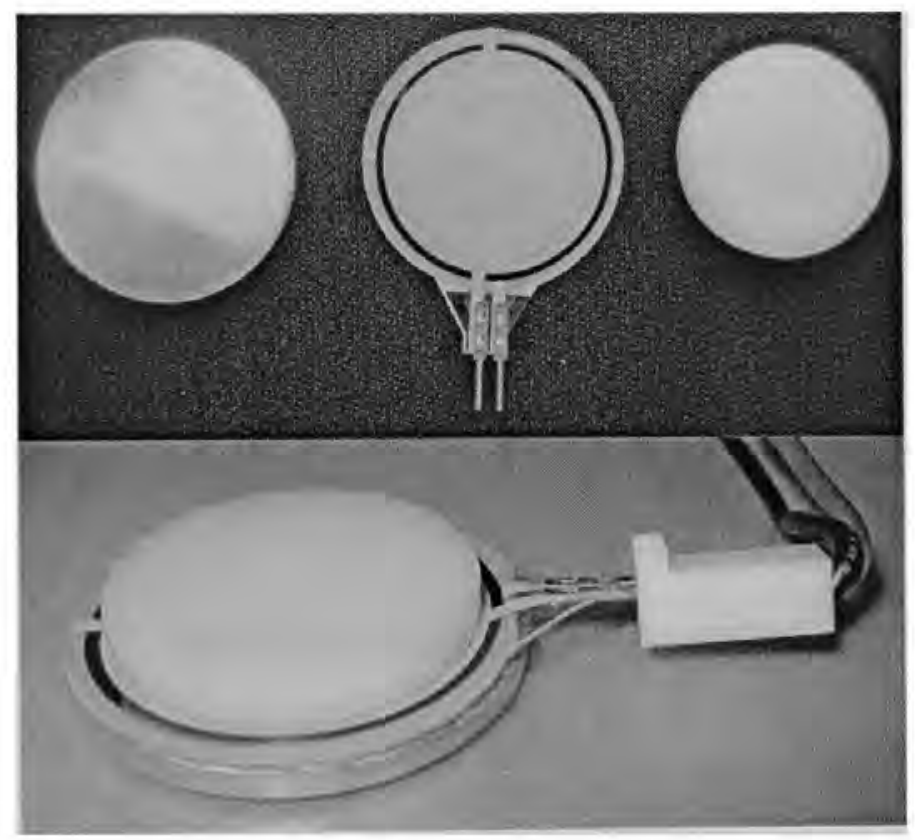

Figure 4.12 Foot switch components and assembly

\subsection{Rotary Incremental Quadrature Encoder}

A rotary incremental quadrature encoder is an electro-mechanical device that converts rotational movement into a digital code signifying angular position. They contain a circular disk with a predefined resolution of radial lines. Two stationary 
sensors that are positioned $90^{\circ}$ out of phase are able to count the number of electrical pulses created by the movement of each line passing over the sensor to determine direction and angular displacement relative to some arbitrary "power-up" starting point. An encoder will be attached to the ankle hinge of the BARA and used to determine the joint angle of the ankle. A light-duty quadrature encoder (Model No. TRD-S1000-BD) from Automation Direct was selected to be used with the BARA. Figure 4.13 displays the schematic diagram of the encoder and Table 4.5 provides the technical specifications of the encoder.

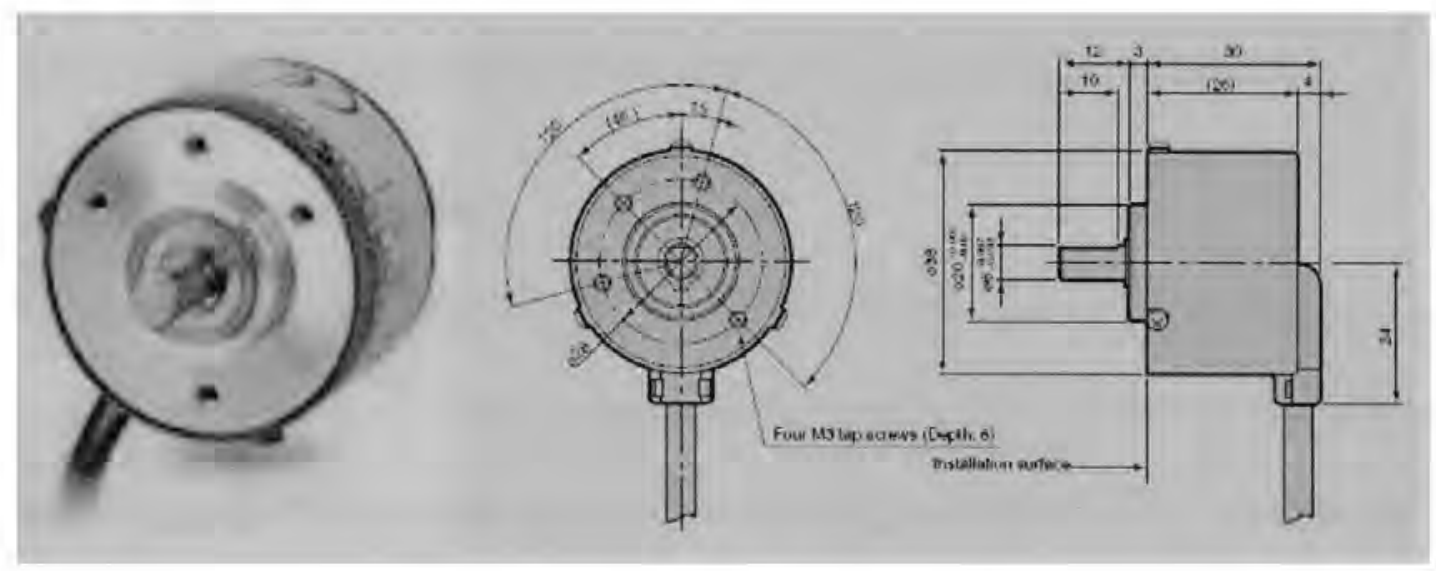

Figure 4.13 Schematic diagram of encoder

\begin{tabular}{|l|l|}
\hline Quadrature Encoder Model TRD-S1000-BD Specification \\
\hline Resolution & $1000 \mathrm{PPR}$ \\
\hline Input Voltage & $12 \mathrm{~V}-24 \mathrm{~V}$ \\
\hline Output & Open Collector \\
\hline Current Consumption & $50 \mathrm{~mA}$ max \\
\hline Weight & $5.3 \mathrm{oz}$ \\
\hline Wiring Schematic & COM \\
\hline Blue & Power supply \\
\hline Brown & Output A \\
\hline Black & Output B \\
\hline White & Output Z \\
\hline Orange & Earth ground \\
\hline Shield &
\end{tabular}

Table 4.5 Encoder specification 


\subsection{Control System}

Similar to how the human body uses the CNS to integrate and issue commands between peripheral organs to coordinate motion, the BARA also utilizes a similar architecture to achieve motion control. The design includes a central processing center which integrates incoming information from peripheral devices. The information is analyzed and based on a preprogrammed logic, specific actions are then communicated outward. There is a host of supporting components within the control system that performs specific and different functions associated with movement. And it requires an intricate network of interconnections between them to achieve the desired result.

\subsubsection{PIC Microcontroller}

A PIC Microcontroller (MCU) is a programmable microprocessor designed for use within an embedded system. They provide specific control functionality and are designed with all the necessary components including an integrated central processing unit (CPU), limited internal memory, and input/output (I/O) ports. The MCU offers a compact and low-cost design solution. The PIC MCU stores custom programs created using various coding languages in a non-volatile memory which can be reused. The PIC MCU (Model No. 16F876-20S/P) manufactured my Microchip was used with the BARA control system. Table 4.6 provides some of the basic specifications of the PIC MCU.

\begin{tabular}{|l|c|}
\hline PIC16F876-20S/P Specification \\
\hline Pin Count & 28 \\
\hline I/O Pins & 22 \\
\hline Architecture & 8 -bit \\
\hline Program Memory Type & Enhanced Flash \\
\hline Program Memory Size & $14 \mathrm{~kb}$ \\
\hline
\end{tabular}

Table 4.6 PIC MCU Specifications 
Creating a custom designed program onto the PIC MCU requires the use of a PC computer, a compatible development environment, a programmer, and a suitable programming language. Figure 4.14 shows the sequence of activities that occur in order to program an $\mathrm{MCU}$.

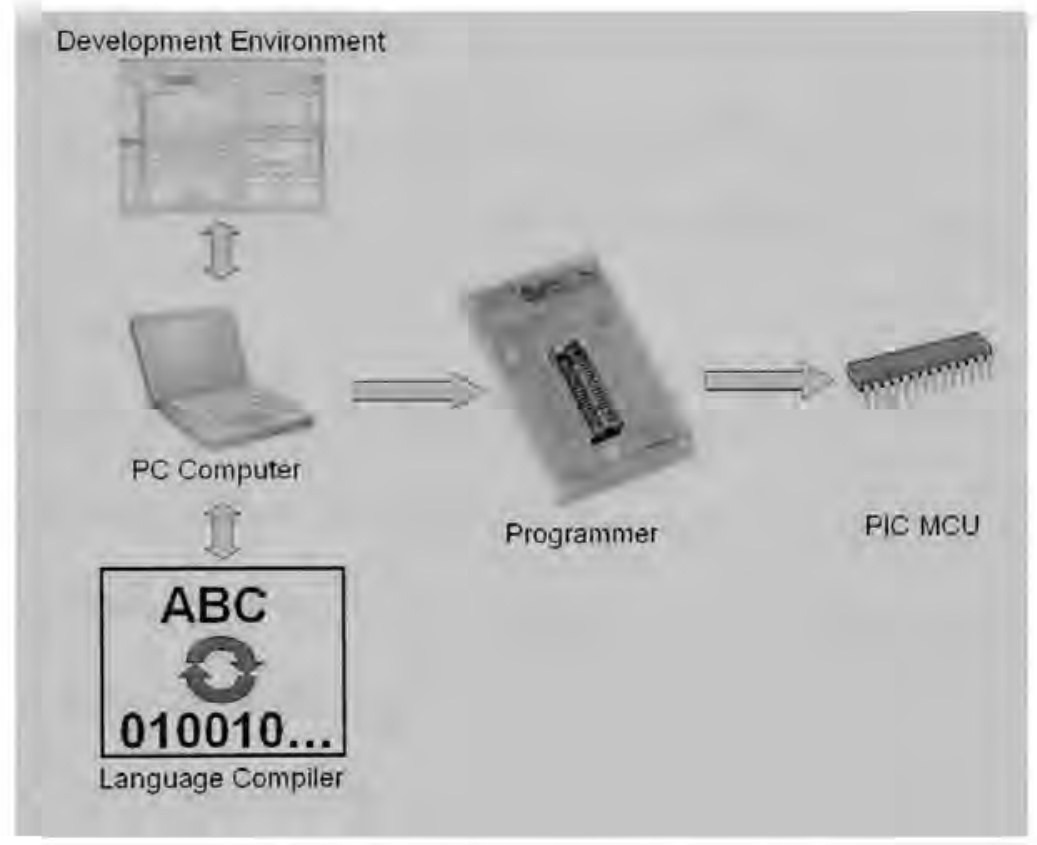

Figure 4.14 Programming sequence

Any PC computers capable of running Windows XP operating system with either a USB or serial port can be used to run the following applications. The MPLAB Integrated Development Environment (IDE) was used as the application development tools for creating the coding language. The development environment provides a usable interface with necessary tools and helpful options that the user can employ to type out programming code. The PicBasic Pro (PBP) Compiler by microEngineering Labs Inc. was selected as the programming language. The PBP language uses short English-like commands similar to the BASIC STAMP II programming language. Once the code is generated on a PC Computer using the development environment, the PBP compiler 
translates the code into a format understandable for use by the PIC MCU. The PICSTART Plus Programmer (Model No. DV003001) manufactured by Microchip was connected to the PC via a USB or Serial port connector cable. The PICSTART Plus is designed with a connector used to easily secure and release the PIC MCU for programming purposes. Once the PIC MCU is properly positioned into the PICSTART Plus, the user is able to begin programming the MCU through the MPLAB IDE application. Once the transfer is successful, the custom code is programmed into the MCU and ready for use.

In order for the BARA device to operate, the PIC MCU must be programmed with a specific functional logic which determines what actions to take given certain inputs. The device can only perform tasks that it is specifically programmed to handle. Therefore figure 4.15 shows the complete logic flowchart that was incorporated into the design of the BARA PIC MCU (see Appendix B for full page image). The flowchart provides a set of directions that instructs the PIC MCU on what actions should be taken given certain input parameters. The square boxes represent tasks or actions that need to be taken. These are similar to efferent signals which require the PIC MCU to generate an output signal directed to some external component. The diamond shaped boxes represent decision points where specific inputs are analyzed and based on the status of the input, different decisions and actions are performed. The flowcharts are used as a guide for writing the algorithmic code used by the PIC MCU. 


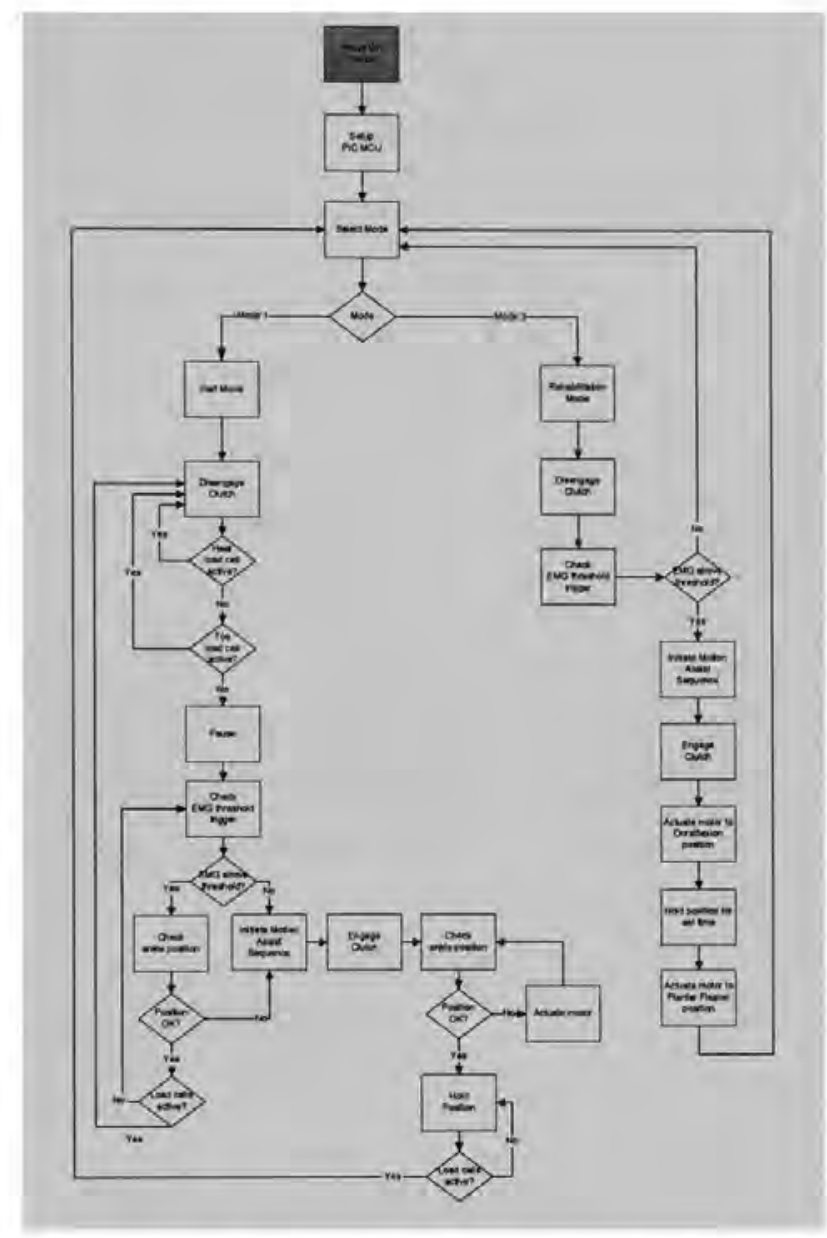

Figure 4.15 Logic flowchart for BARA (see Appendix B for full size image)

Coding standards typically follow a useful format where the complete code is comprised of a compilation of individual modules. Each module is created to perform a specific task with a limited functional scope. The modules are linked through certain command calls within the code. This allows the user to efficiently save memory space, provide a more helpful format when trying to identify bugs, and the ability to reuse modules without duplicating the code. The BARA uses a similar compilation of task specific modules in its program. Figure 4.16 provides an outline block diagram showing the various modules used within the program (See Appendix $\mathrm{C}$ for the complete PBP code). 


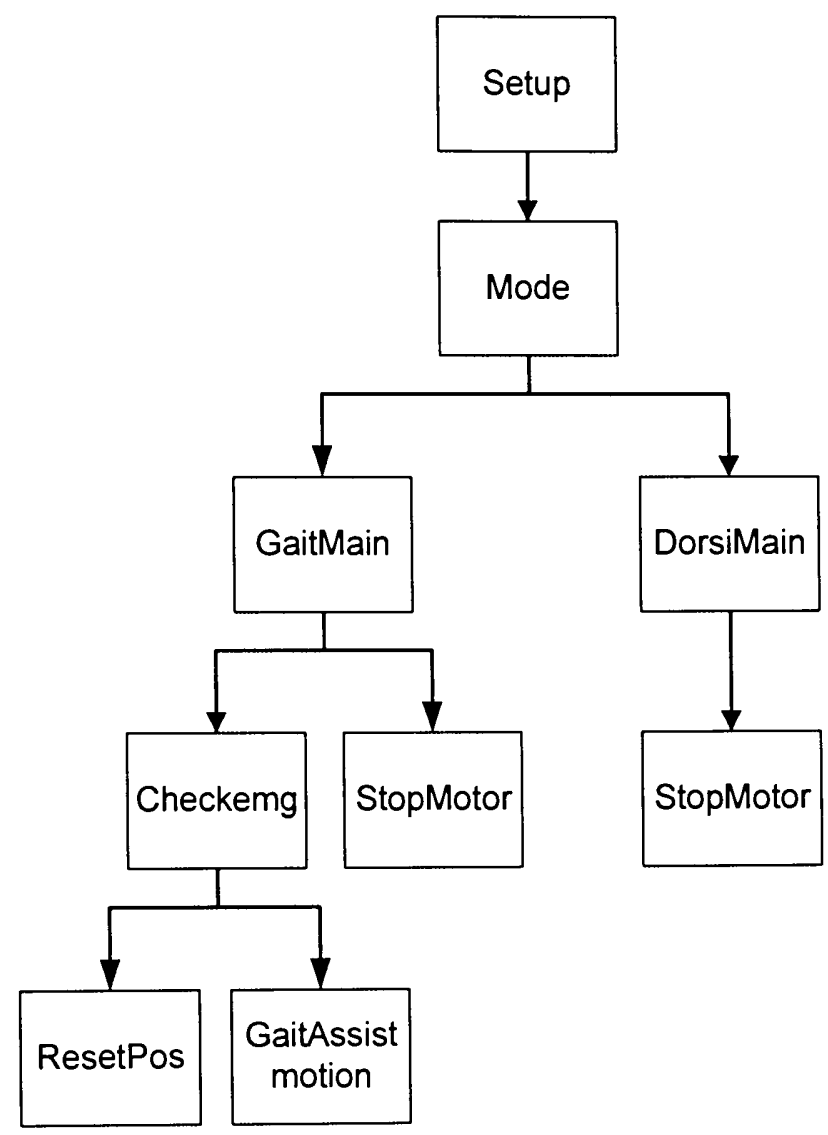

Figure 4.16 BARA Program Modules

The "Setup" module initiates a sequence of actions necessary to properly configure both the PIC MCU as well as the PIC Servo SC controller when the device is powered-up. It performs the following instructions,

- Sets the address of PIC Servo SC

- Defines the formats the information packets

- Reset the encoder to the neutral position

- Defines the PID filter parameters

- Resets variable values

The Setup module is followed by the Mode module. 
The "Mode" module reads the input from the training mode circuit switch to determine whether the device will be used for dorsiflexion rehabilitation training or gait rehabilitation training. The PIC MCU will illuminate the appropriate LED to indicate which mode the device has been set to. This module is linked to the GaitMain and DorsiMain modules.

The "GaitMain" module represents the first set of instructions for the gait rehabilitation training. This module incorporates both foot switches (i.e. Heel load cell and Foot load cell) as inputs to determine when the foot makes contact with the ground. When either of the inputs is active, no subsequent action is taken. The PIC MCU illuminates the appropriate LED representing the active input. A pause of $400 \mathrm{~ms}$ is used following the activation of the Heel load cell to insure no action is taken after heel strike. This insures the device will not trigger prematurely in the case the heel load cell sensor looses input during the progression of the stance phase where free motion about the ankle is required. A pause of $200 \mathrm{~ms}$ is added following the activation of the Foot load cell to allow a window of opportunity for the user to manually move their ankle into the proper neutral position during the swing period prior to the device making automatic adjustments. The Checkemg and StopMotor modules are called upon within this module.

The "StopMotor" provides a simple set of code used to instruct the motor controller to stop all motion of the motor and remain in state of waiting. This allows the motor to respond efficiently to the next set of instructions when necessary.

When the "Checkemg" module is called, the PIC MCU reads the input signal from the EMG biofeedback device to determine if the user is voluntarily attempting to dorsiflex their ankle into the neutral position. If the EMG signal is triggered, the BFAFO 
will issue a command to the PIC SERVO SC to return the current position of the encoder. The PIC MCU will then compare the current position to the preset neutral position. If the angular difference exceeds $\sim 4^{\circ}$ off neutral, the device will automatically initiate movement to correct the difference. If no EMG signal is detected, the BARA will also automatically initiate movement to position the ankle into the proper neutral position. If either of the foot switches becomes activated during this time signifying the stance period, the program will automatically return to the GaitMain module where the clutch is released to allow free motion. This module calls upon the ResetPos and GaitAssitmotion modules.

The "ResetPos" module is a simple set of code used to reset variables used within the program to zero. These variables hold temporary values that are used to calculate and compare value when called upon. Resetting the variable prevents cumulative addition of variable values from occuring allowing accurate results whenever the variable is used.

When the "GaitAssist" module is called upon, the PIC MCU will issue an immediate command to activate the electromagnetic clutch to couple the motors output to the ball screw. The PIC MCU will issue a subsequent command to the PIC SERVO SC to initiate rotation of the motor back to the neutral ankle position. Again if either of the foot switches becomes activated during this time signifying the stance period, the program will automatically return to the GaitMain module where the clutch is released to allow free motion.

The "DorsiMain" module is the secondary training mode available for use by the BARA. This module when called releases the clutch to allow the user to manually move their ankle. This module also incorporates the input from the EMG device to see when 
the signal is strong enough to trigger the sequence. Once triggered, the PIC MCU sends one signal to engage the electromagnetic clutch. A second command is issued to the motor control system to actuate the ankle to approximately $16^{\circ}$ dorsiflexion. The position is held for 3 seconds and the ankle it automatically returned to a position of $\sim 25^{\circ}$ plantar flexion. The device resets awaiting the next EMG trigger or change in the training mode.

\subsubsection{Motion Controller}

A motion controller is a typical automations tool composed of a variety of electronic circuitry used to control the speed and/or position of machinery. Motion controllers typically generate desired positional output signals which are transmitted to an actuator such as an electric motor; and utilize a closed feedback loop such as an encoder to achieve an accurate end position. They play an important part in the control of robotics and $\mathrm{CNC}$ machines.

The PIC-Servo SC (Part No. KAE-T0V10-BDV1) by Jeffrey Kerr, LLC (Figure 4.17-a) was chosen as the motion controller board for the BARA. The PIC-Servo SC is designed specifically for use in controlling DC brush-type motors utilizing incremental encoder feedback. It allows for trapezoidal profiling utilizing customizable velocity and acceleration settings in addition to inputting PID filter gain values. The PIC-Servo SC connects to the motor and encoder via a DB15 connection. The PIC-Servo SC connects to the PIC MCU using a z232-485 serial port converter (Part No. KAE-Z232485-BDV2), through a RS232 port (Figure 4.17-b). 


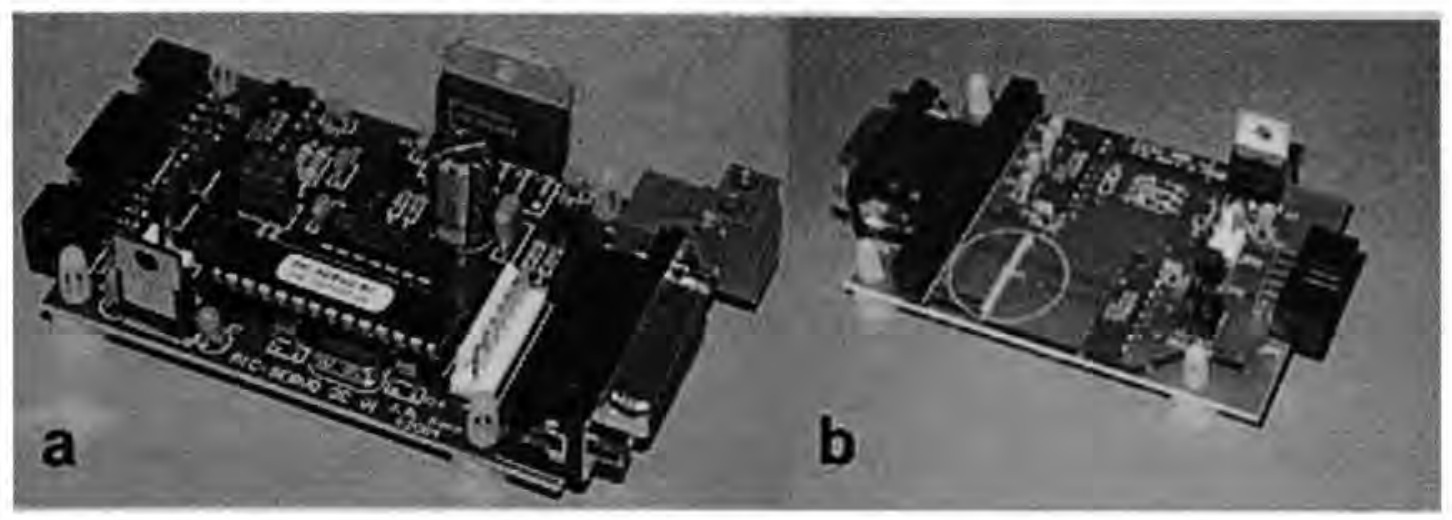

Figure 4.17 PIC-Servo SC and z232-485 serial port converter

This requires a 10-pin ribbon cable to interconnect the z232-485 with the PIC-Servo SC.

Table 4.7 provides specifications of the PIC-Servo SC and Z232-485 Serial Port Converter.

\begin{tabular}{|l|l|}
\hline PIC-Servo SC Specification \\
\hline Motor Type & DC Servo Motor (brush type) \\
\hline Driving Current Rating & 3A cont./6A peak \\
\hline Driving Voltage rating & 12-48VDC \\
\hline Motor/Encoder Connector & DB15 Male \\
\hline Network Connector & 10-Pin flat ribbon IDC Socket \\
\hline Nominal Dimension & 2.1"x 3.1"x 0.9" \\
\hline Z232-485 Serial Port Converter Specifications \\
\hline Logic Power & 7.5V-12V DC \\
\hline RS232 Connector & DB9 Female \\
\hline Dimension & 2.1" $x 3.1$ "x 0.9" \\
\hline
\end{tabular}

Table 4.7 PIC-Servo SC and Z232-485 Serial Port Converter Specifications

\subsubsection{Circuitry Design}

The control circuit is the integration of the various electrical components interconnected into a single cohesive element to perform a predefined operation. Figure 4.18 provides an illustration of the overall system architecture for the BARA design. The 
design incorporates the foot switches, EMG input, electromagnetic clutch, DC motor, and encoder and integrates them to the PIC MCU.

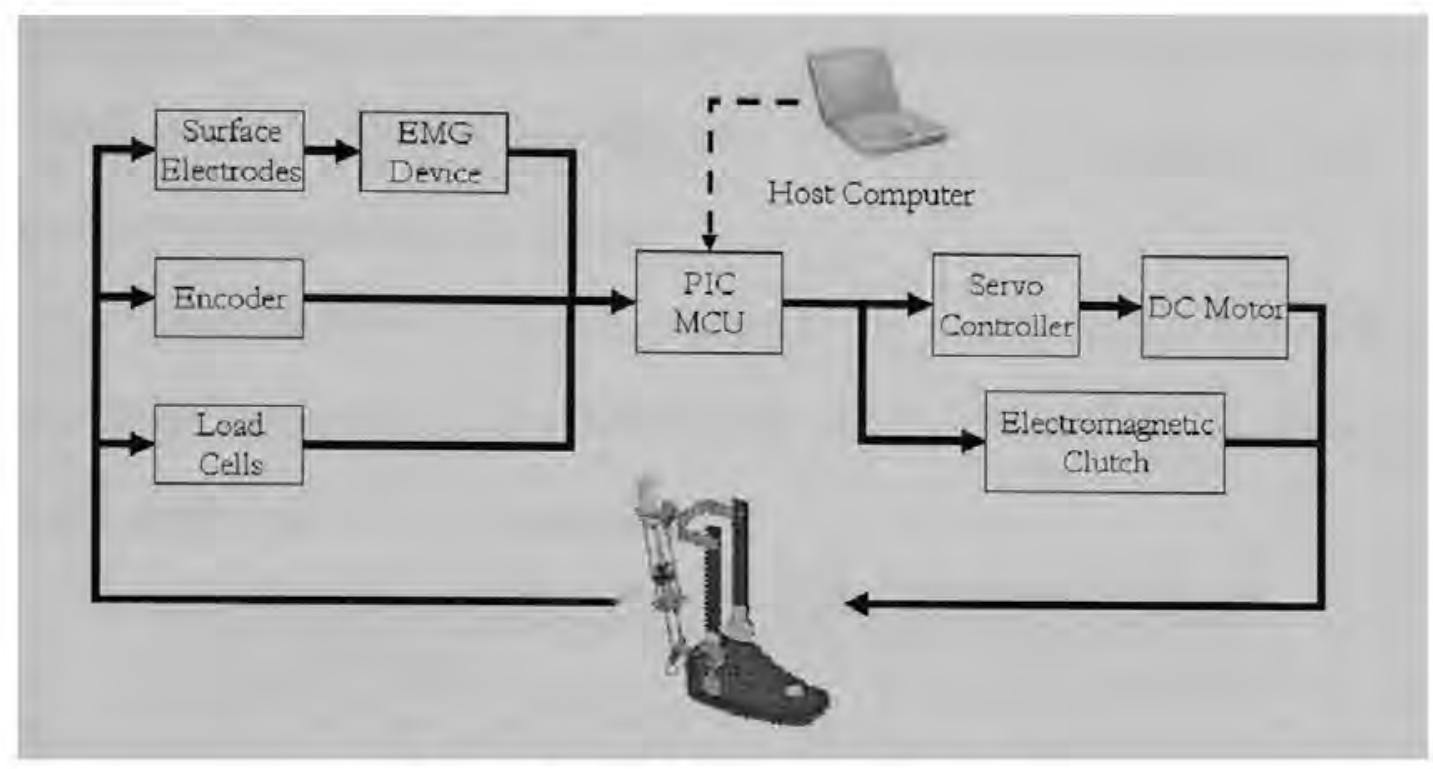

Figure 4.18 BARA System architecture

The center of the BARA control circuit architecture is the PIC MCU which serves as the primary integration device for receiving inputs and issuing output commands. The design of the control circuit requires the use of 12 of the PIC MCU pins as I/O ports.

Table 4.8 provides a mapping of the PIC MCU I/O pins.

\begin{tabular}{|c|c|l|}
\hline Pin No. & Input/Output & \multicolumn{1}{|c|}{ Source/Target } \\
\hline 2 & Input & Heel load cell \\
\hline 3 & Input & Foot load cell \\
\hline 4 & Output & Heel active LED indicator \\
\hline 5 & Output & Foot active LED indicator \\
\hline 17 & Output & RS232 Transmit \\
\hline 18 & Input & RS232 Receive \\
\hline 21 & Output & Electromagnetic clutch \\
\hline 22 & Input & EMG device trigger signal \\
\hline 23 & Output & EMG active LED indicator \\
\hline 24 & Input & Rehabilitation mode switch \\
\hline 25 & Output & Dorsiflexion rehabilitation mode LED indicator \\
\hline 26 & Output & Gait rehabilitation mode LED indicator \\
\hline
\end{tabular}

Table 4.8 PIC MCU Pin I/O Mapping 
The control circuit architecture is composed of six separate circuits. Each circuit performs a specific task related to the function of the BARA. The six circuits are (1) the Power supply circuit, (2) the PIC MCU circuit, (3) the Electromagnetic clutch circuit, (4) the EMG circuit, (5) the Foot switch circuit, and (6) the Motion control circuit. See Appendix D for complete circuit diagram.

The various electrical components comprising the BARA require three different voltage levels for proper operation (i.e. $5 \mathrm{~V}, 9 \mathrm{~V}, 12 \mathrm{~V}$ ). Table 4.9 provides the specific voltage requirements for the components.

\begin{tabular}{|l|c|}
\hline \multicolumn{1}{|c|}{ Component } & Voltage requirement \\
\hline PIC 16F876 MCU & $5 \mathrm{~V}$ \\
\hline LT 1761 RS232 Driver/Receiver IC & $5 \mathrm{~V}$ \\
\hline TSH22 Operational Amplifier IC & $5 \mathrm{~V}$ \\
\hline Flexiforce load sensor & $5 \mathrm{~V}$ \\
\hline 4N31 Optoisolator IC & $5 \mathrm{~V}$ \\
\hline Rehabilitation mode switch & $5 \mathrm{~V}$ \\
\hline Z232-485 Serial Port Converter & $9 \mathrm{~V}$ \\
\hline SO-11 Electromagnetic clutch & $12 \mathrm{~V}$ \\
\hline PIC-Servo SC & $12 \mathrm{~V}$ \\
\hline
\end{tabular}

Table 4.9 Electrical component voltage requirement

The Power circuit manages the voltage output generated by the AC-DC switching power supply to provide the correct voltage levels needed (Figure 4.19). The 12V power supply can be used directly from the AC-DC switching power supply. The $5 \mathrm{~V}$ and $9 \mathrm{~V}$ supplies require the use of voltage regulators to step down the voltage output. The LM7805 voltage regulator provides the $5 \mathrm{~V}$ output. The LM7808 voltage regulator generates the $9 \mathrm{~V}$ output using two additional resistors as shown. 


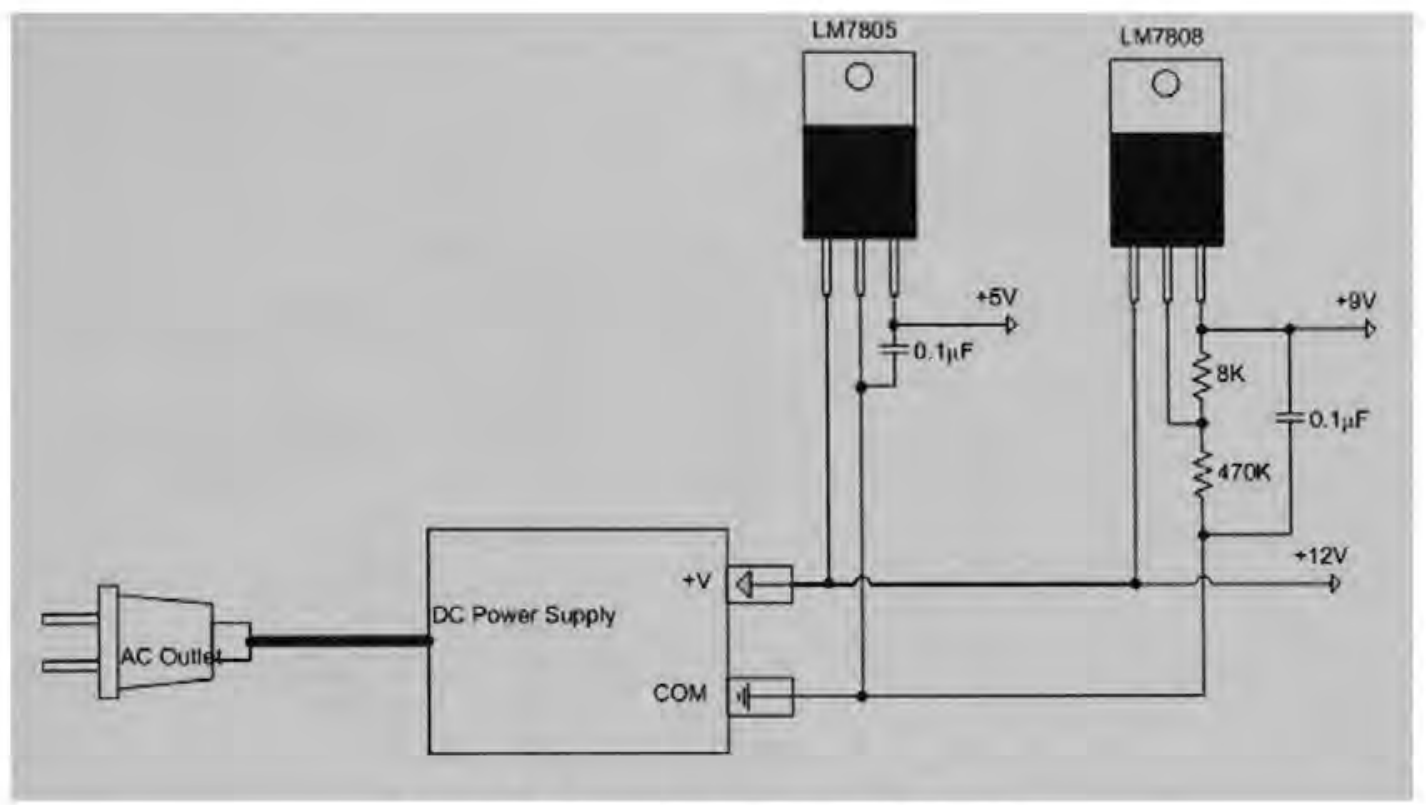

Figure 4.19 Power supply circuit schematic

The PIC MCU requires some basic connections for the circuit to function properly. Figure 4.20 provides the schematic for setting up the PIC MCU. The PIC MCU requires a $5 \mathrm{~V}$ power supply connected to pins 1 and 20 , along with pins 8 and 19 connected to COM. The PIC also uses a $12 \mathrm{MHz}$ ceramic resonator for the internal timing. The rehabilitation mode switch is designed to provide a High input (i.e. $\sim 5 \mathrm{~V}$ ) when the switch is open and inversely a Low input (i.e. $\sim 0 \mathrm{~V}$ ) when the switch is closed. The PIC MCU has five LEDs connected which are used to indicate when certain inputs/outputs are active, such as the foot switches, the EMG trigger, and the current rehabilitation mode. 


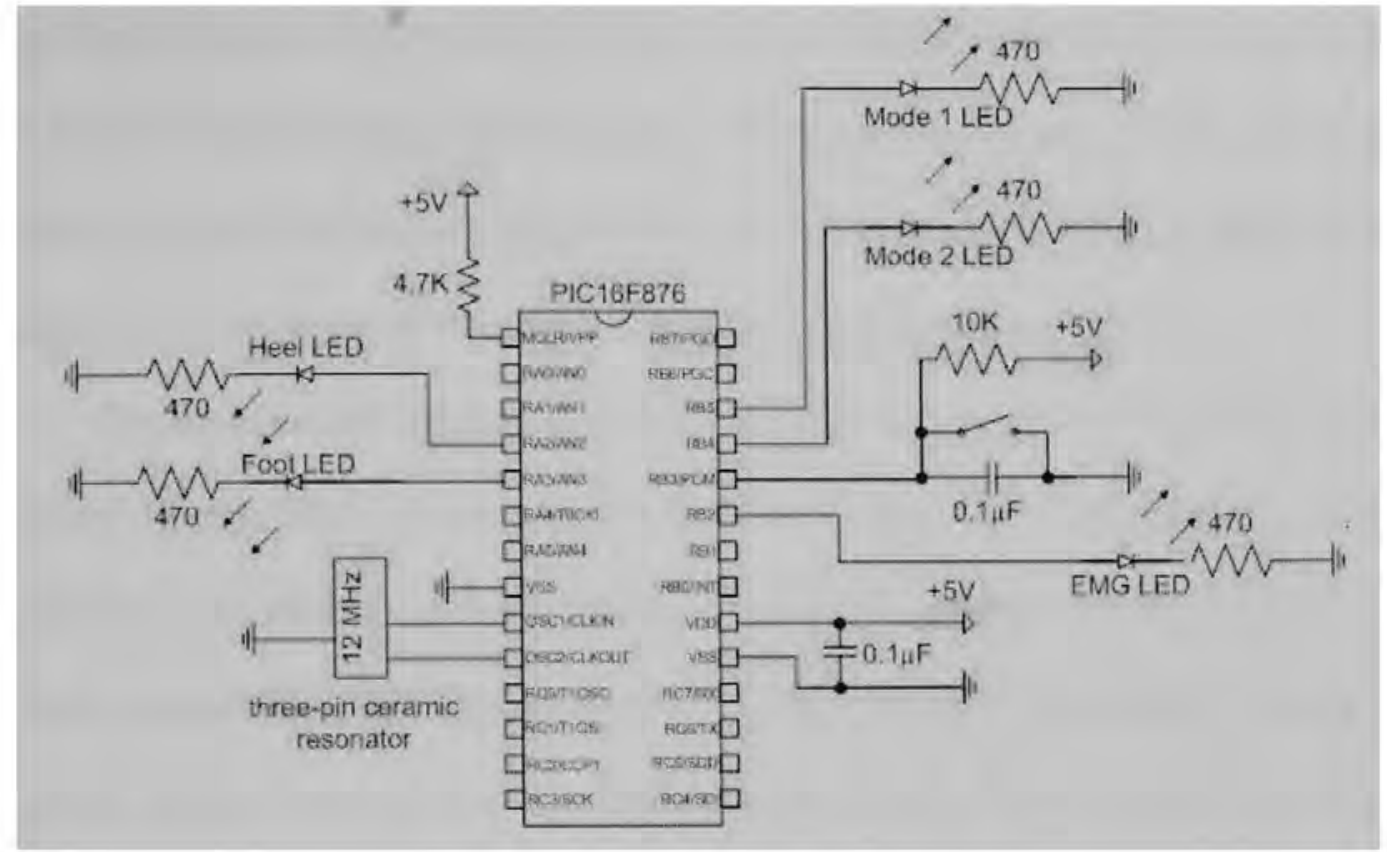

Figure 4.20 PIC MCU circuit schematic

The electromagnetic clutch circuit is used as an output for the PIC MCU to activate the clutch assembly. It utilizes a solid state relay which acts similar to a transistor by providing a source of amplification while still isolating the original signal from the clutch assembly to eliminate noise and interruption (Figure 4.21).

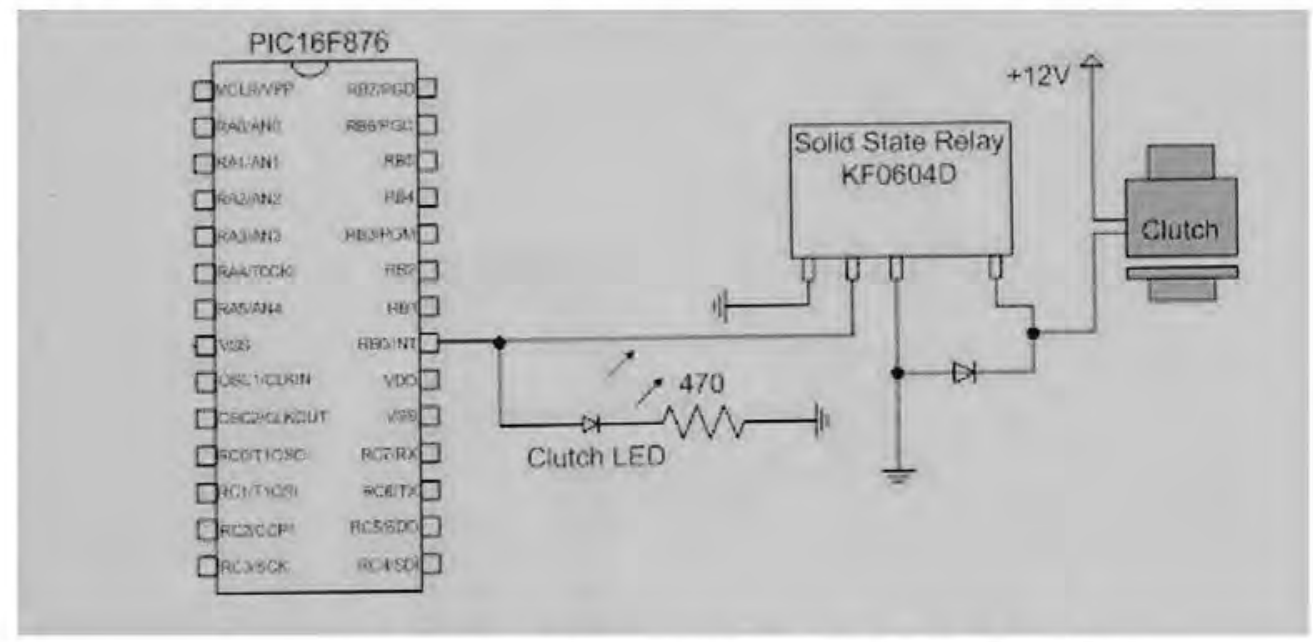

Figure 4.21 Electromagnetic clutch circuit schematic 
When the PIC output is Low, the solid state relay is closed preventing the higher voltage to flow thus inactivating the clutch system. When the PIC output is High, this triggers the relay to open thus activating the clutch. An LED is connected in parallel with the output pin to serve as an indicator as to when the clutch assembly is active.

The EMG circuit required the use of an Optoisolator (Part No. 4N31-NTE3084) IC Circuit (Figure 4.22). Connecting the EMG device directly into the circuit resulted in interference that affected the performance of the EMG device. The Optoisolator circuit optically isolates the EMG output from the PIC MCU input by allowing the voltage from the EMG output to activate an LED within the optoisolator. The activated LED triggers the optoisolator to allow the $5 \mathrm{~V}$ power supply to reach the PIC MCU input pin.

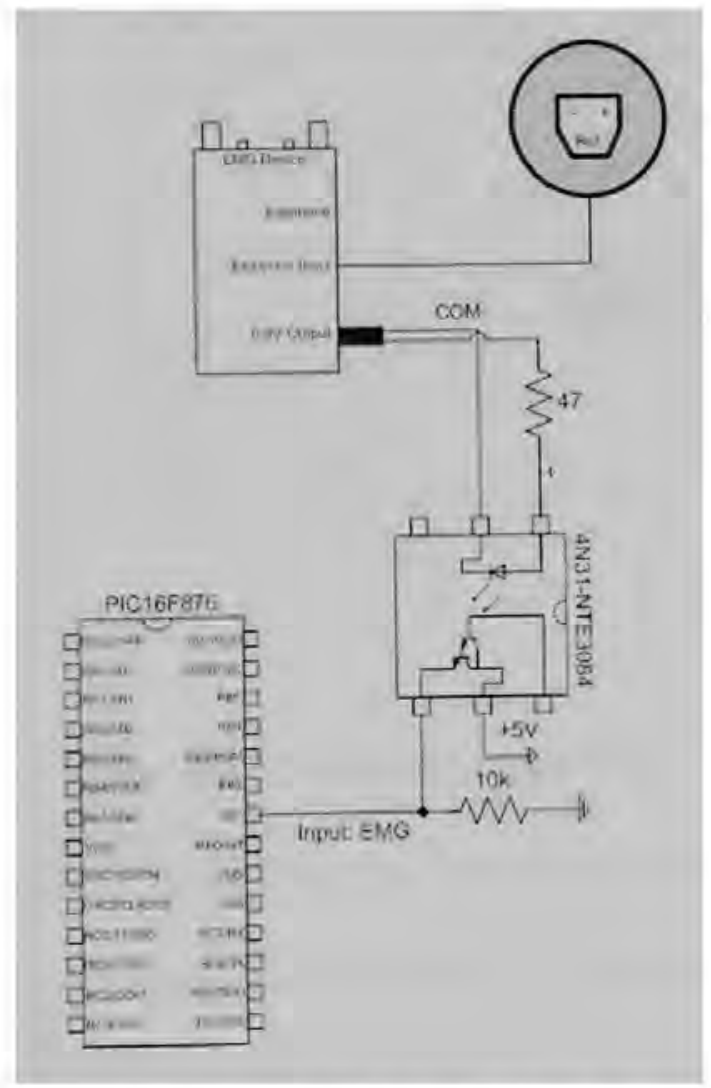

Figure 4.22 EMG circuit schematic 
The foot switch circuit utilizes a dual Op-Amp (Part No. TSH22) IC circuit in addition to two $500 \mathrm{~K}$ potentiometers, and two load sensors (Figure 4.23). The potentiometers serve as the reference resistor used to adjust the sensitivity of the load sensors. The potentiometers can be adjusted from $0-500 \mathrm{~K} \Omega$. The Op-Amp serves as a comparator between the two inputs. When the reference resistance is equal to the load sensor resistance, the Op-Amp triggers an output of $5 \mathrm{~V}$ which serves as the input signal to the PIC MCU. The $5 \mathrm{~V}$ serves as a High inputs signal signifying a loading response representative of the stance period. This triggers a series of actions based on the PIC MCU program.

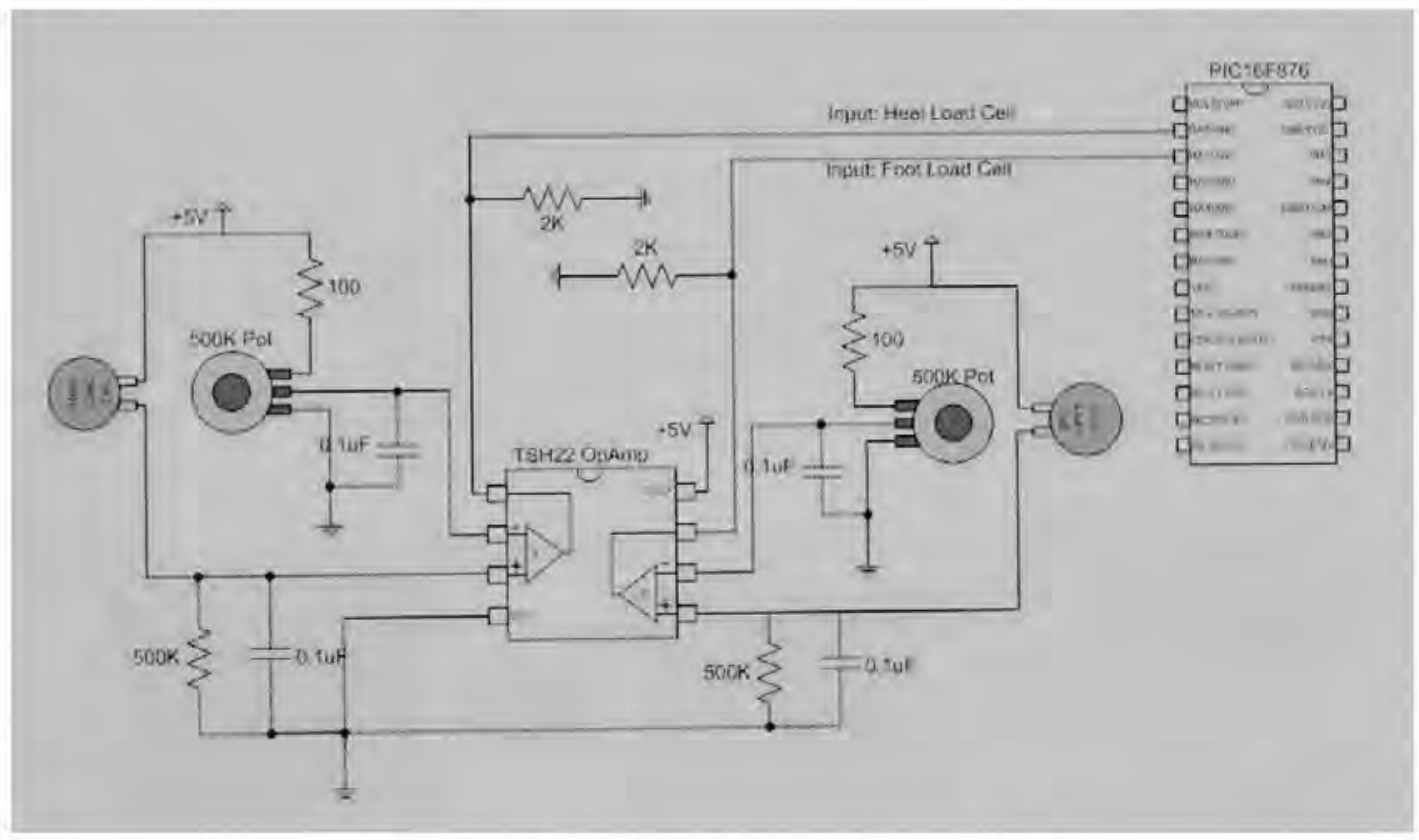

Figure 4.23 Foot switch circuit schematic

The Motion control circuit involves the integration of the servo controller with the PIC MCU. The peripheral components include the DC motor, quadrature encoder, the PIC-SERVO SC board, the Z232-485 serial converter board, and a LT1781 RS232 Dual 
Driver/Receiver IC (Figure 4.24). The encoder and DC motor are wired directly to the PIC-Servo SC board via a DB15 pin connector. The PIC-Servo SC and Z232-485 are networked using a 10-pin flat ribbon cable. The Z232-485 communicates through the RS232 connector to the LT1781 utilizing a DB9 pin connector. Table 4.10 provides the pin mapping for the DB15 and DB9 connector. The LT1781 generates the necessary RS232 voltage signals which relay incoming and outgoing packets of information between the PIC MCU and the PIC-Servo SC motion control boards.

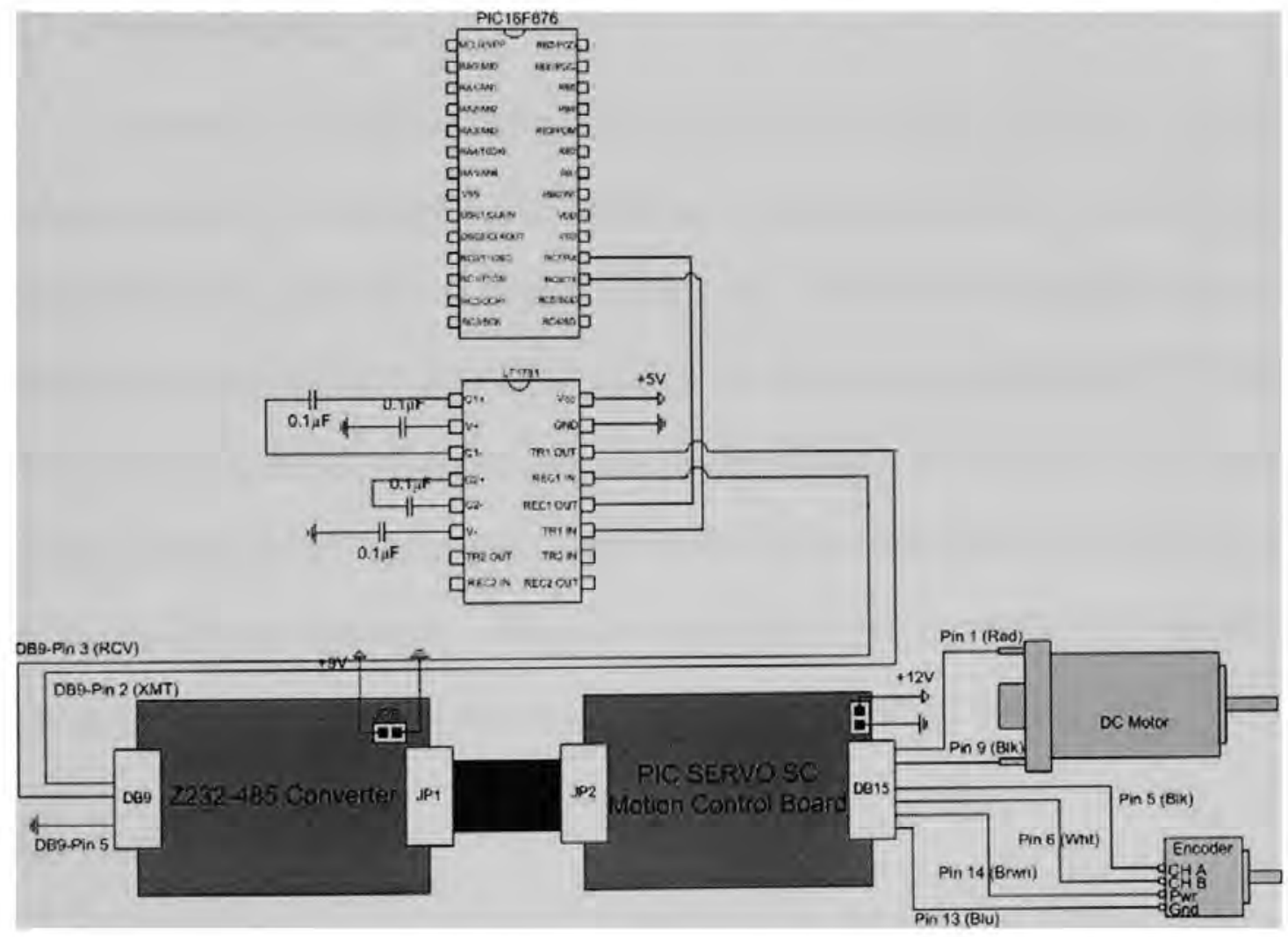

Figure 4.24 Motion control circuit schematic 


\begin{tabular}{|l|l|l|}
\hline \multicolumn{3}{|l|}{ DB15 Pin Connector } \\
\hline Pin 1 & Motor Output (M+) & Red wire \\
\hline Pin 5 & Encoder Channel A & Black wire \\
\hline Pin 6 & Encoder Channel B & White wire \\
\hline Pin 9 & Motor Output (M-) & Black wire \\
\hline Pin 13 & Encoder COM & Blue wire \\
\hline Pin 14 & Encoder power supply & Brown wire \\
\hline DB9 Pin Connector & Red wire \\
\hline Pin 2 & XMT- & Green wire \\
\hline Pin 3 & RCV+ & Black wire \\
\hline Pin 5 & COM &
\end{tabular}

Table 4.10 DB15 and DB9 pin mapping

\subsection{Power Supply}

An AC-DC Switching power supply is required to supply the necessary amount of voltage and current to all the electrical components powering the BARA. The Meanwell Enclosed Switching DC Power Supply (Model No. S-100F-12) was chosen because it allows the BARA device to draw power from a standard Alternating Current (AC) outlet. This provides a readily available and near endless supply of electricity. The power supply converts the AC signal into a stable Direct Current (DC) electrical supply for use by the circuitry components. The power supply provides a portable unit capable of producing the required $12 \mathrm{~V}$ output and a maximum current of 8.5 Amps.

\subsection{System Integration}

The creation of the BARA system required the final assembly of the numerous mechanical and electrical components. 


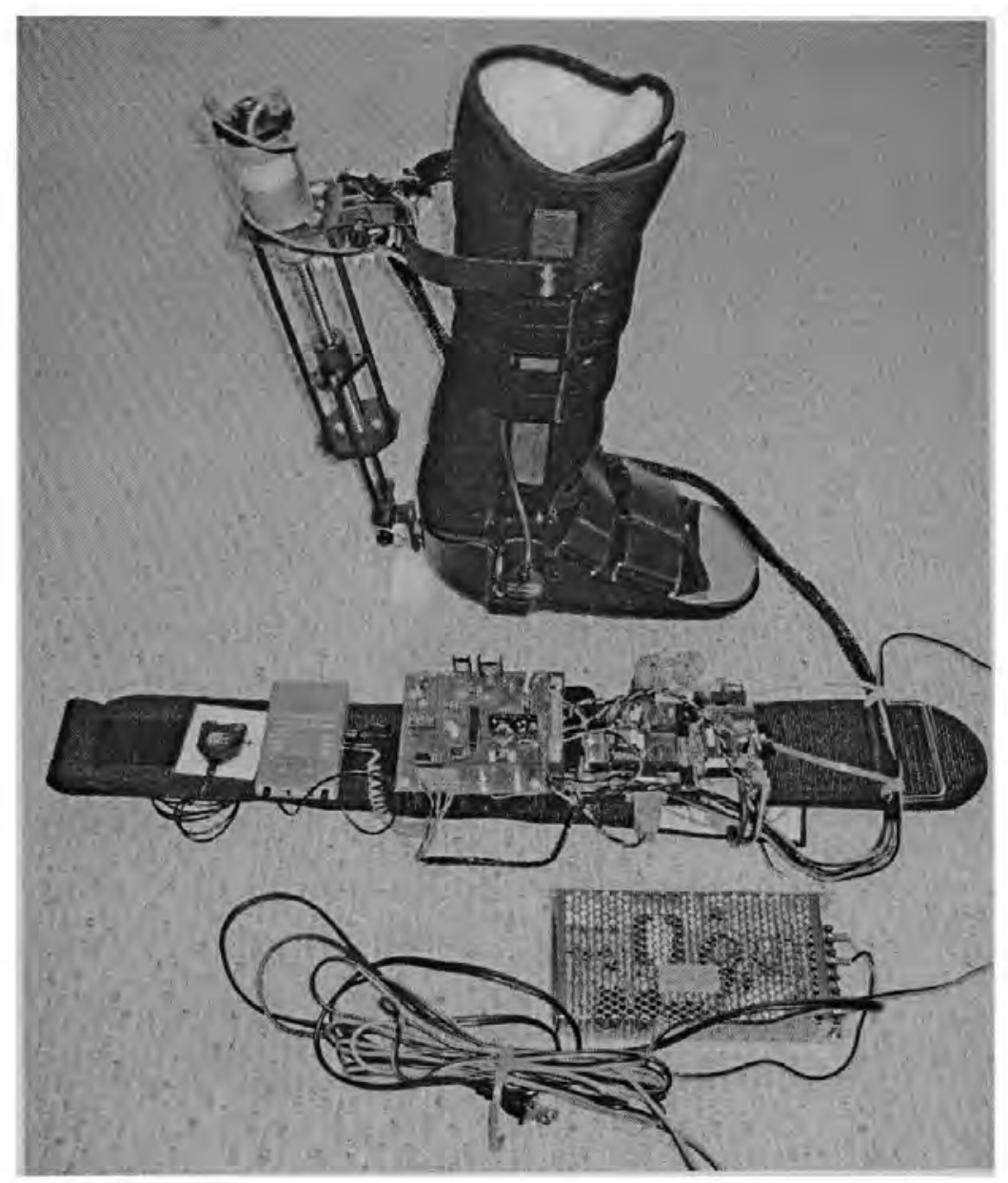

Figure 4.25 Final assembly of BARA

The BARA device consists of two sections consisting of (1) the control system circuit boards, and (2) the mechanically actuated AFO brace. Figure 4.25 shows a picture of the complete BARA system. The control circuit was created using a soldering protoboard (Part No. 263250) from Jameco Electronics. The protoboard was cut to a dimension of 4.5in $\mathrm{x}$ 5in. The IC's, resistors, capacitors, and LED's used within the design was assembled onto various sized IC sockets which was then positioned onto the protoboard and soldered into position. The IC sockets provided an easy mean of removing/replacing any component that may be necessary due to damage. 


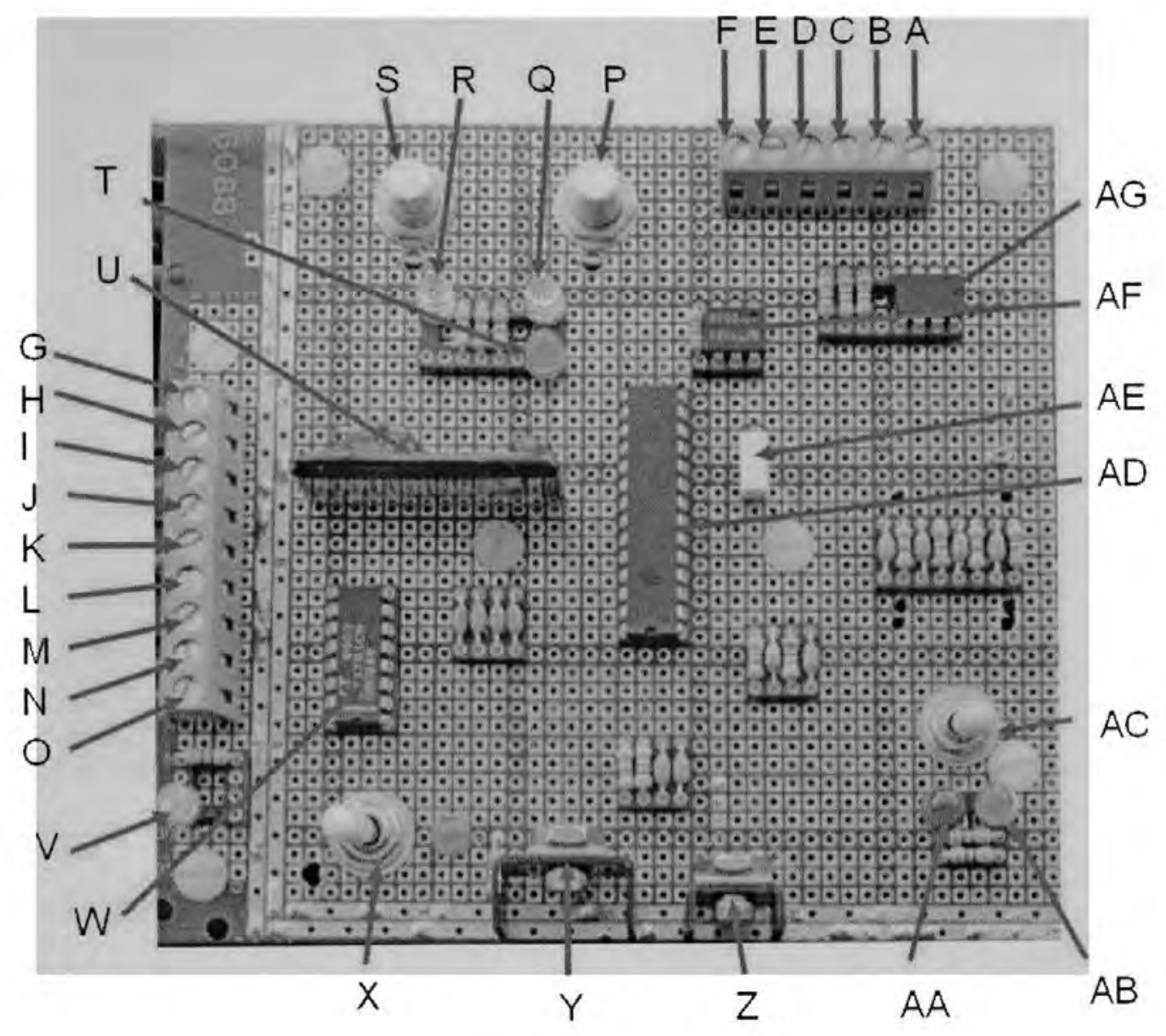

Figure 4.26 Control Circuit Board

Figure 4.26 shows the final control circuit with the various components described in Table 4.11. A combination of $20 \& 30$-gauge wiring was used to connect the electronic components running through the underside of the prototype circuit board. Heats sinks were also added to the two voltage regulators to help dissipate the heat generated. 


\begin{tabular}{|c|l|c|l|}
\hline Item & \multicolumn{1}{|c|}{ Description } & Item & \multicolumn{1}{|c|}{ Description } \\
\hline A & Terminal 1 & R & Foot LED indicator \\
\hline B & Terminal 2 & S & Foot sensor adjuster \\
\hline C & Terminal 3 & T & EMG LED indicator \\
\hline D & Terminal 4 & U & Solid state relay \\
\hline E & Terminal 5 & V & Clutch LED indicator \\
\hline F & Terminal 6 & W & RS232 Dual Driver/Receiver IC \\
\hline G & Terminal 7 & X & Power switch and LED \\
\hline H & Terminal 8 & Y & Voltage regulator (9V) \\
\hline I & Terminal 9 & Z & Voltage regulator (5V) \\
\hline J & Terminal 10 & AA & Dorsiflexion mode LED indicator \\
\hline K & Terminal 11 & AB & Gait mode LED indicator \\
\hline L & Terminal 12 & AC & Rehabilitation mode selector \\
\hline M & Terminal 13 & AD & PIC MCU \\
\hline N & Terminal 14 & AE & Ceramic resonator \\
\hline O & Terminal 15 & AF & Optoisolator \\
\hline P & Heel sensor adjuster & AG & Operational Amplifier \\
\hline Q & Heel LED indicator & & \\
\hline
\end{tabular}

Table 4.11 Control Circuit Board components

Terminals blocks were positioned around the perimeter of the circuit board to provide easier access and a secure means of connecting the various peripherals to the $\mathrm{I} / \mathrm{O}$ ports of the PIC MCU, certain IC's, as well as supplying power to various components.

Table 4.12 provides the I/O mapping of the 15 terminal connections.

\begin{tabular}{|c|l|c|l|}
\hline Terminal \# & \multicolumn{1}{|c|}{ Description } & Terminal \# & \multicolumn{1}{|c|}{ Description } \\
\hline T1 & Heel Load Cell - lead 1 & T9 & PIC-Servo SC (+12V) \\
\hline T2 & Heel Load Cell - lead 2 & T10 & PIC-Servo SC (COM) \\
\hline T3 & Foot Load Cell - lead 1 & T11 & Z232-485 (+9V) \\
\hline T4 & Foot Load Cell - lead 2 & T12 & Z232-485 (COM) \\
\hline T5 & EMG Input - COM & T13 & RS232 - COM (Pin 5) \\
\hline T6 & EMG Input - +V & T14 & RS232 - XMT- (Pin 2) \\
\hline T7 & Clutch - lead 1 & T15 & RS232 - RCV+ (Pin 3) \\
\hline T8 & Clutch - lead 2 & & \\
\hline
\end{tabular}

Table 4.12 Terminal block I/O map

20-gauge wiring was used to connect the peripheral $\mathrm{I} / \mathrm{O}$ components to the terminal blocks. 
The control circuit board is then mounted to a $1 / 8$ " thick plastic sheet cut to size to serve as a rigid backing. Seven holes were drilled through open areas in the circuit board. Plastic rods of length $5 / 8$ " were cut to serve as supporting columns to hold-up the circuit board and protect the wiring underneath. Both ends were drilled and threaded for attaching screws. Plastic screws were used to secure the control circuit board to the plastic backing. Similar backings were creating for both the PIC-Servo SC and Z232-485 serial port converter boards. The circuit boards were also secured using the plastic screws. The Control circuit, PIC-Servo SC, and Z232-485 boards were connected in line using 1.25 " steel hinges. A velcro strip was adhered onto the back of the plastic backing for each component to be used later to attach the circuit boards to the utility belt.

Because the BARA will be used as an ambulatory device, the entire control system was also designed to be easily portable. All four electronic components (i.e. control circuit, PIC-Servo SC, z232-485, and EMG device) were attached to a utility belt using velcro strips which could then be worn around the users waist. An Atlus weight lifting belt was used for the purpose of the utility belt which provided a 4" wide surface area with velcro attachments included; and provided enough area for attaching the four components.

The power supply is the only stationary component of the BARA. An 18-gauge wire was used to connect the power supply to the control system to provide the $12 \mathrm{~V}$ output necessary to power the electronic components. A wire length of $25^{\prime}$ was used for this connection which provides the BARA with a walking range of approximately 50 '.

The BARA brace required the final assembly of the four main components which are (1) the brace connectors, (2) the actuator, (3) the encoder, and (4) the foot switches. 
Table 4.13 provides a list of the individual components comprising the BARA. Appendix E provides an exploded view rending of both the actuator with all the subcomponents as well as the complete BARA brace. Figure 4.27 shows the actual device assembled. The encoder is secured to the encoder mount. The shaft of the encoder is connected to the encoder cap and secured in place using a set screw.

\begin{tabular}{|c|c|}
\hline \multicolumn{2}{|c|}{ Brace connectors } \\
\hline & Pro-Rom Walker \\
\hline & Calf connector \\
\hline & Calf connector hinge \\
\hline & Heel connector hinge \\
\hline \multicolumn{2}{|c|}{ Actuator } \\
\hline & DC motor \\
\hline & Clutch sleeve \\
\hline & Electromagnetic clutch \\
\hline & Ball screw \& ball bearing assembly nut \\
\hline & Bearing housing \\
\hline & Actuator support frame \\
\hline & Actuator piston frame \\
\hline \multicolumn{2}{|c|}{ Encoder Assembly } \\
\hline & Quadrature encoder \\
\hline & Encoder cap \\
\hline & Encoder mount \\
\hline \multicolumn{2}{|c|}{ Foot Switches } \\
\hline & Heel load cell \\
\hline & Foot load cell \\
\hline
\end{tabular}

Table 4.13 Acuator components list

This design allows the encoder body to rotate as the ankle moves relative to the encoder shaft which stays stationary to provide the angular displacement measurements. The two load switches are held in position by velcro strips on the foot base. They are positioned about the heel and ball of the users' foot. The wiring connecting the DC motor, electromagnetic clutch, encoder, and two load cells are routed to the control system using approximately 4-6' of cabling. 


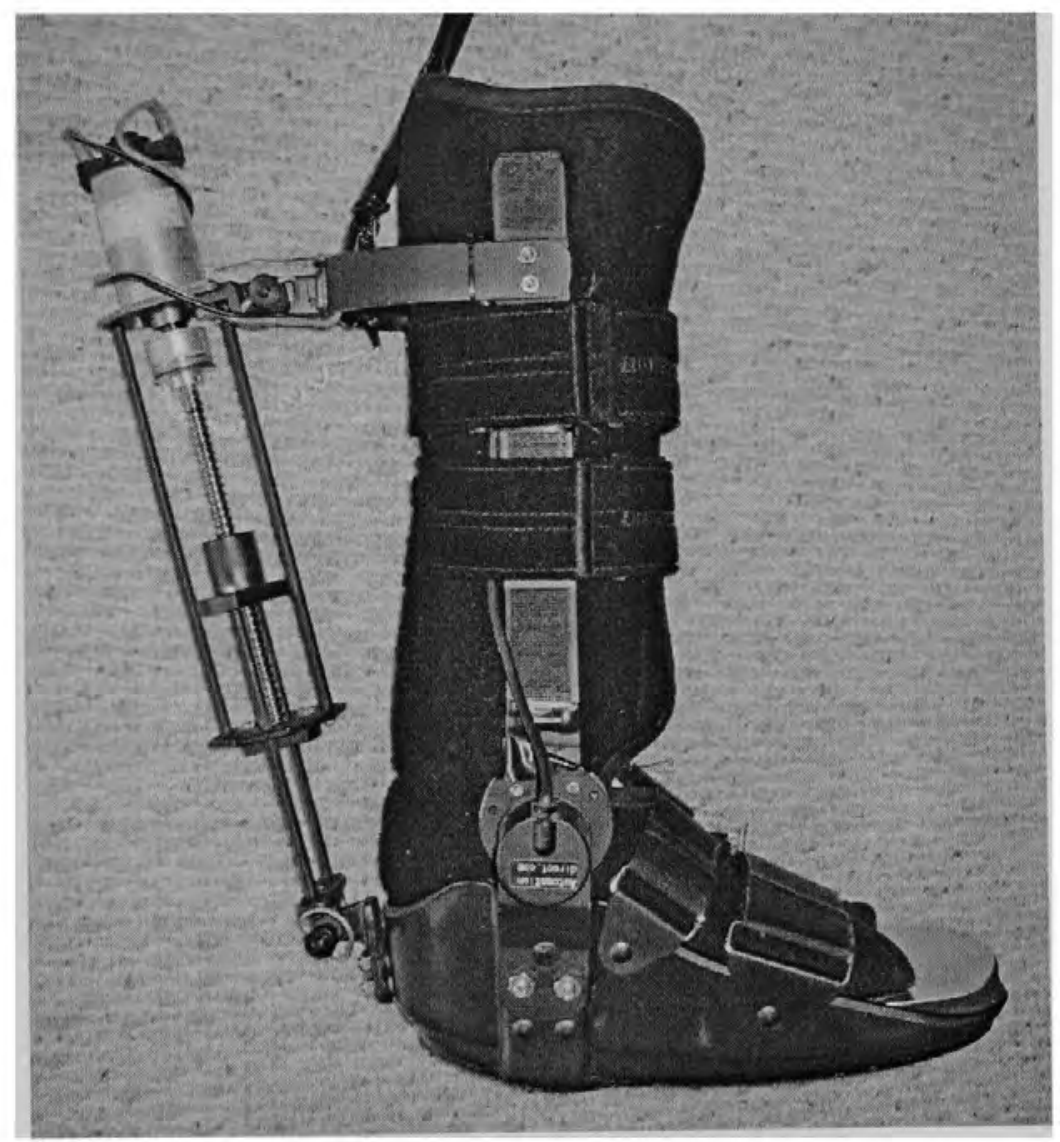

Figure 4.27 Image of BARA assembled 


\section{EXPERIMENTAL PROCEDURES}

\subsection{Video Motion Capture}

The kinematic analysis of the gait experiment requires the use of a video camera (Model No, JVC GR-DVL9800), a lighting system, passive reflective markers, a computer system, and the APAS (Ariel Performance Analysis System) software. The video camera will record the walking motion of the subject and transfer the video images into the computers memory. The passive reflective markers are small spherical shaped markers $(\sim 0.75 \mathrm{in}$. dia.) with a light reflective surface (Figure 5.1$)$. The reflective markers will be strategically positioned onto the user and background. The lighting system provides a source of illumination that will reflect off the markers resulting is a high contrast distinguishing mark on the video image.

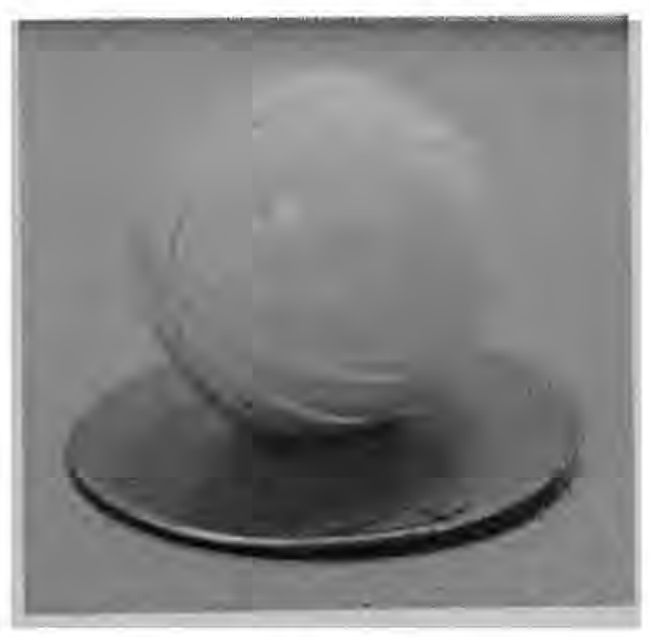

Figure 5.1 Passive Reflective Marker

The APAS software is a video-based 3-D motion analysis system. The software includes all the tools necessary to digitize the video image, process the information, and 
provide quantitative data and displays results illustratively. The APAS software will capture video images at a rate of 60 frames per second (fps).

\subsubsection{Calibration Frame}

The APAS system requires the configuration of a calibration frame image that is used by the software to determine relative distance. A rectangular steel frame was used which includes four reflective markers located at the corners of the structure representing the four control points. The frame measures $20 \mathrm{~cm}$ wide $\times 40 \mathrm{~cm}$ high providing the relative position in space of the four control points (Figure 5.2). The calibration frame was positioned on the ground along the walking path in front of the video camera. Several frames of video were taken and stored to the computers memory.

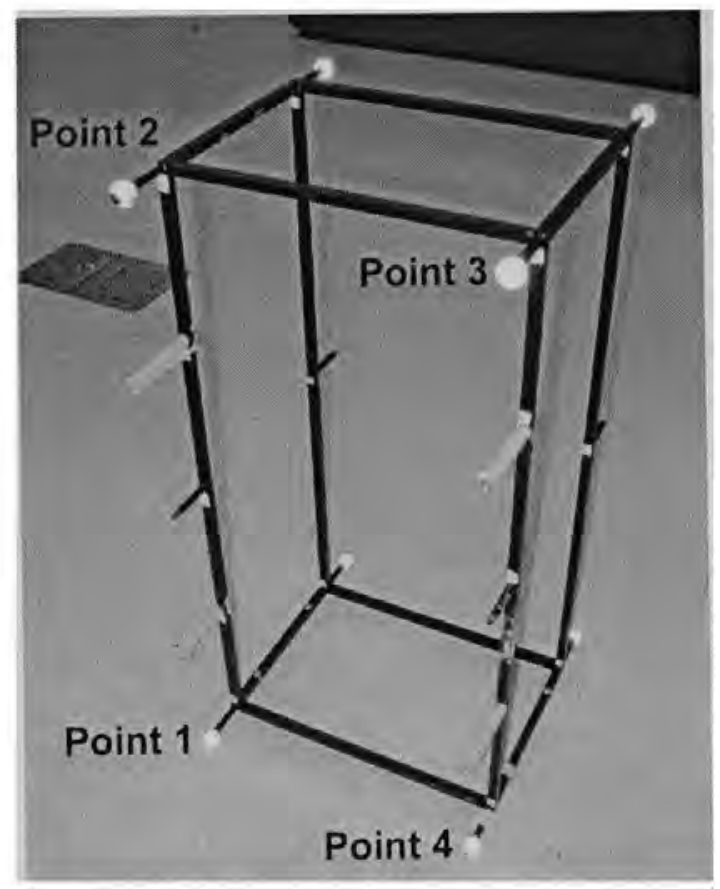

Figure 5.2 Calibration Frame 


\subsubsection{Marker Placement}

The passive reflective markers are used to provide (1) a fixed point of reference on the video image, and (2) relative positions of joints and limbs on the human body. Four markers will be used for this experimental setup. One marker will be positioned as a stationary object in the background. This will provide any information on the relative movement of the camera in space while filming. The remaining three markers will be attached to the side of the right leg facing the camera on both the human subject and BARA brace. The three limb markers will represent the (1) leg, (2) ankle, and (3) foot of the user. The markers are positioned in a way so that when the user is in a straight standing position, the markers will have a relatively linear alignment (Figure 5.3).

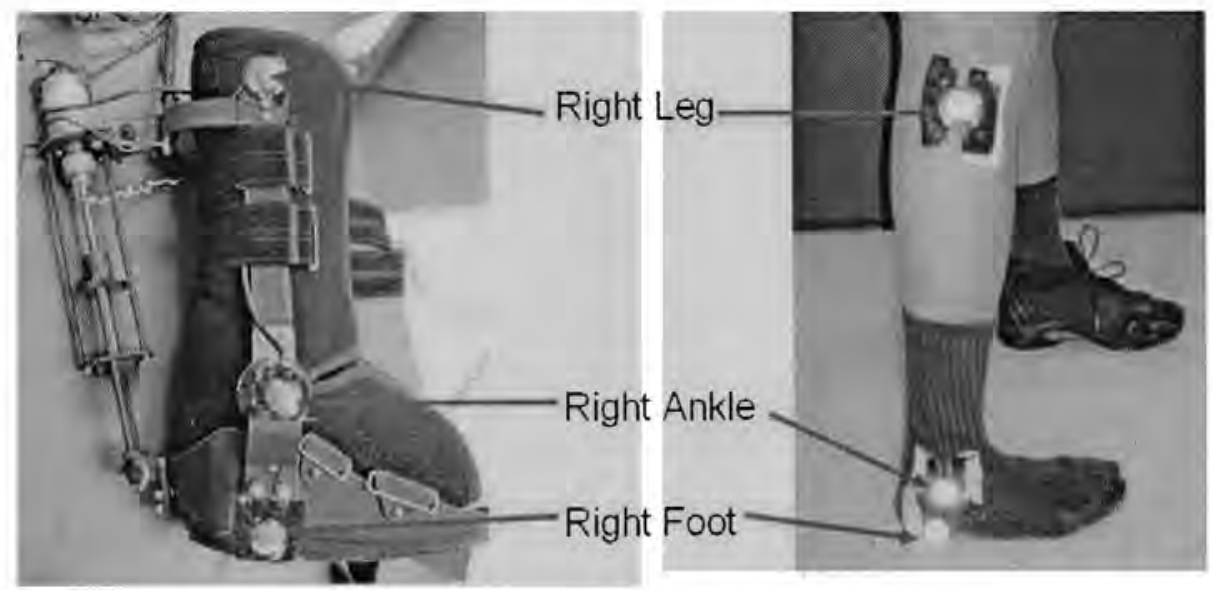

\begin{tabular}{|l|l|l|}
\hline Marker & \multicolumn{1}{|c|}{ BARA } & \multicolumn{1}{c|}{ Subjects Leg } \\
\hline Leg & Top center of AFO support bar & Head of the Fibula \\
\hline Ankle & Center of encoder body & Lateral Malleolus \\
\hline Foot & $\begin{array}{l}\text { Bottom center of ankle hinge } \\
\text { support on the foot base }\end{array}$ & $\begin{array}{l}\text { At the base of the foot } \\
\text { directly below the Lateral } \\
\text { Malleolus }\end{array}$ \\
\hline
\end{tabular}

Figure 5.3 Reflective marker placement 


\subsection{Experimental Setup}

\subsubsection{Equipment Setup}

The experiment was conducted in the Gait Analysis laboratory at Florida International University's Physical Therapy department. The video camera and lighting equipment was setup as shown in Figure 5.4. The video camera was positioned approximately $14^{\prime}$ from the walking path to allow a wide enough camera view to capture a minimum of one complete gait cycle. An area $7 \mathrm{ft}$. in length was marked off and centered directly in front of the camera view. This gait cycle frame provided the ideal area for performing the complete gait cycle of the right leg. The staring position for the subject was approximately $7 \mathrm{ft}$. behind the gait cycle frame.

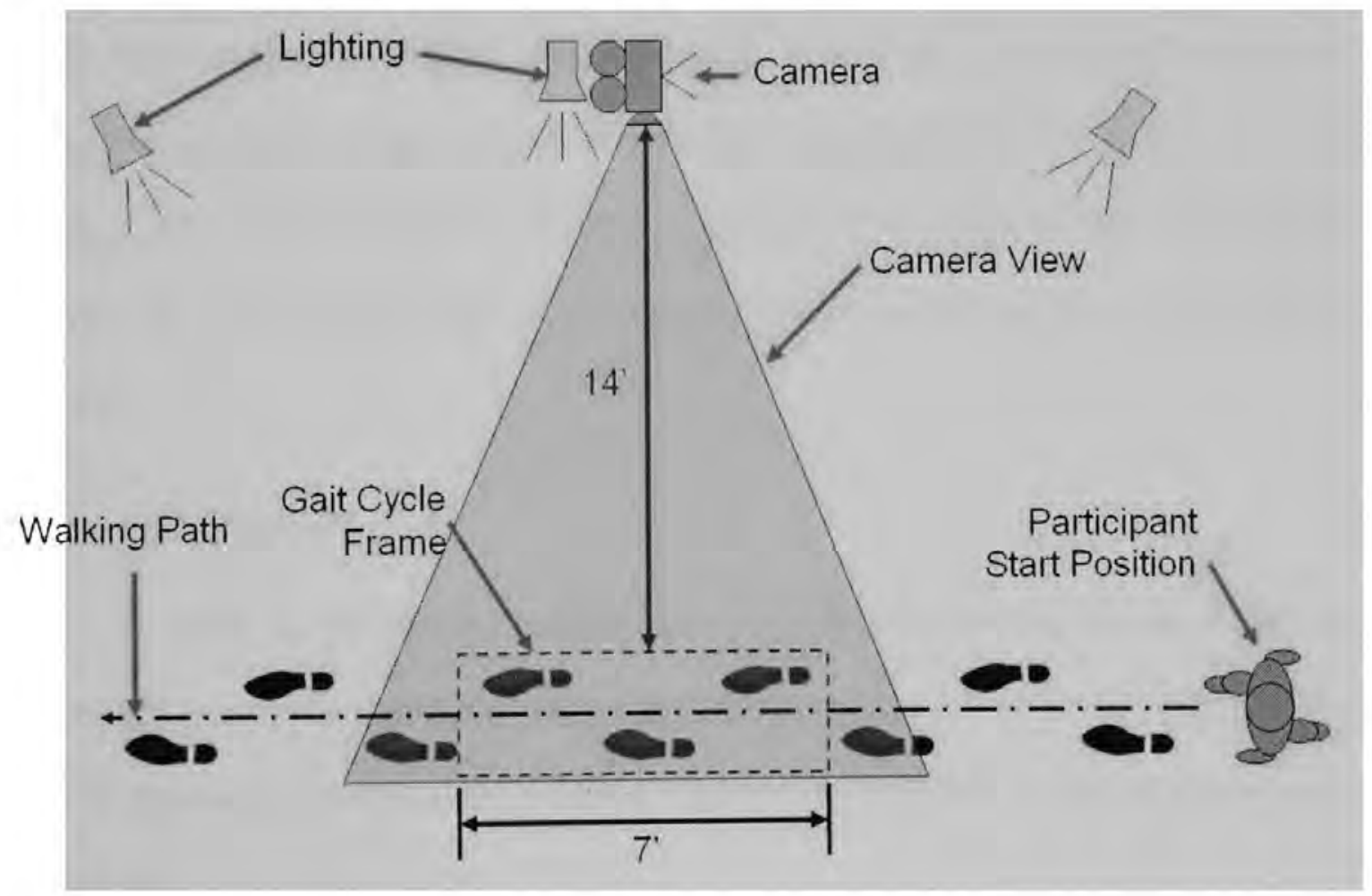

Figure 5.4 Video motion capture equipment layout 
This allows the user at least one complete gait cycle to achieve regular walking patterns prior to entering the gait cycle frame. The subject would follow a straight walking path and continue their walking cycle for at least three steps after exiting the gait cycle frame.

Prior to turning on the BARA device, the user must be in a comfortable straight upright standing position. This standing position is typically the user's neutral ankle position when the foot and lower leg are near perpendicular to one another. This neutral position is representative to the perpendicular ankle position achieved during the swing phase of gait to allow proper foot clearance. Once turned-on, the BARA will initiate its preliminary setup procedures which include setting the current ankle position as the desired neutral reference position.

Once the device is activated, both the heel and foot switches should be adjusted to fit the user's weight requirement. The two potentiometer dials on the control circuit will adjust the sensitivity of the foot switches to match the force the user exerts onto the load sensors. The sensors should be adjusted to provide the minimal amount of weight to activate the foot switches while still remaining inactive once the foot is lifted off the ground.

\subsubsection{Gait Scenario}

In order to provide comparison data into the effectiveness of the BARA at improving joint ankle movement during gait, five scenarios are performed requiring the user to demonstrate different gait patterns. Table 5.1 details the various scenarios to be conducted. 


\begin{tabular}{|c|c|c|}
\hline Gait Scenario & Description & \# Trials \\
\hline Barefoot-Normal Gait & $\begin{array}{l}\text { - User is barefoot } \\
\text { - Perform normal walking patterns }\end{array}$ & 3 trials \\
\hline $\begin{array}{l}\text { Barefoot-Simulated } \\
\text { Drop-Foot Gait }\end{array}$ & $\begin{array}{l}\text { - User is barefoot } \\
\text { - Simulate paralysis of the right } \\
\text { dorsiflexor muscle exhibiting a flaccid } \\
\text { muscle tone during swing phase }\end{array}$ & 3 trials \\
\hline BARA-Normal Gait & $\begin{array}{l}\text { - User will don the BARA } \\
\text { - The device will remain unpowered } \\
\text { - Perform natural walking patterns }\end{array}$ & 3 trials \\
\hline $\begin{array}{l}\text { BARA-Simulated Drop- } \\
\text { Foot Gait }\end{array}$ & $\begin{array}{l}\text { - User will don the BARA } \\
\text { - The device will remain unpowered } \\
\text { - Simulate paralysis of the right } \\
\text { dorsiflexor muscle exhibiting a flaccid } \\
\text { muscle tone during swing phase }\end{array}$ & 3 trials \\
\hline BARA-Assistive Gait & $\begin{array}{l}\text { - User will don the BARA } \\
\text { - The device will be powered-up and } \\
\text { activated } \\
\text { - Simulate paralysis of the right } \\
\text { dorsiflexor muscle exhibiting a flaccid } \\
\text { muscle tone during swing phase }\end{array}$ & 3 trials \\
\hline
\end{tabular}

Table 5.1 Experimental Gait Scenarios

Prior to the start of the experiment, the user is given approximately 30 minutes to wear the BARA while unpowered and allowed to walk on a level surface to become familiar with the feel of the device. In addition, the user is allowed approximately 10 minutes to practice simulating drop-foot gait patterns. Both practice sessions allow the user to become familiar with the changes in gait pattern to prevent falling or injury.

In order for the APAS software to recognize the subject's neutral position, two calibration videos must be acquired with the user standing in a static upright straight position; one with the user barefoot and the second with the BARA device worn. Using the video motion capture setup, the user is asked to stand in the center of the gait cycle frame with the right side facing the camera. Two seconds of video is recorded for each standing calibration and stored into memory. 
Based on the nature of the study being a preliminary validation study using only a single subject test setup, a comprehensive statistical analysis will not be conducted. Therefore each gait scenario will be repeated only three times sequentially to collect multiple data sets for general comparison analysis. The orders in which the gait scenarios are conducted are not considered. A five second recording length is used to capture each gait pass. This provides enough time for the user to completely pass through the entire camera frame during the walking sequence. After each pass, the user is allowed enough time to return to the starting position and prepare for the next sequence.

\subsection{Data Analysis}

The APAS software includes a toolset of five modules that are used to perform the majority of kinematic analysis of the video files which are the (1) Trimmer, (2) Digitize, (3) Transform, (4) Filter, and (5) Display. The Trimmer module is used to edit the video file by cropping the section of video that contains only the video frames of interest. For this research, this will include the complete timeframe when the subject's right leg remains in view of the camera frame to provide the maximum amount of kinematic data on the gait pattern. The timeframe indicator is synchronized to zero when the right leg makes the initial heel strike contact to the floor. This will indicate the start time of the gait cycle. All frames before this time marker will be tracked as a negative time value.

Once the video is cropped, the Digitize module is used to calibrate the frame of the video and identify the limb markers to be processed. The coordinates of the four calibration control points are entered into the program along with the three marker points 
representing the lower leg. The researcher will begin on the first frame of the video file and manually label the three markers points as being the right leg, right ankle, and right foot. This will create a 2-D stick diagram representing the leg and foot pivoting about the ankle point (Figure 5.5). The software will apply the same marker labels to the remaining frames of the video based on internal algorithm which identify the reflective pixels in the video file.

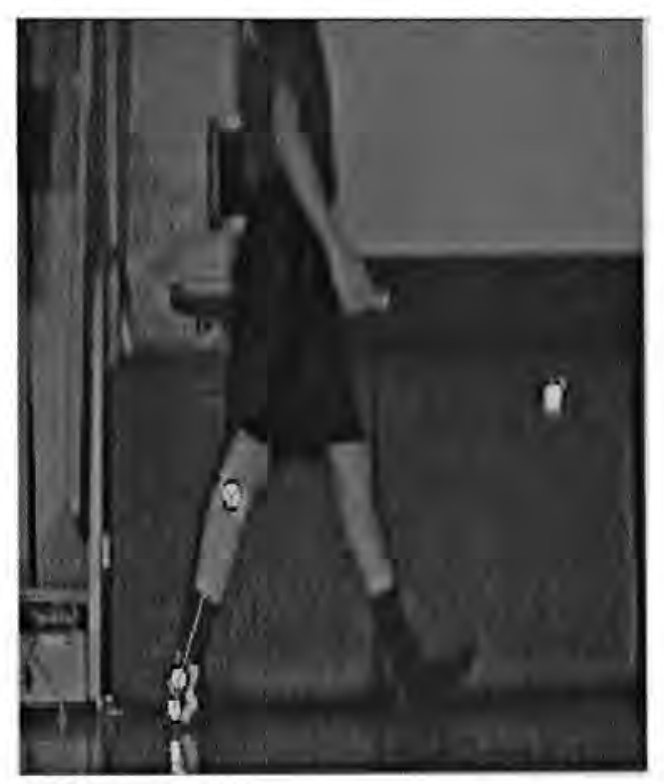

Figure 5.5 APAS Digitize Module

The Transform module uses internal computational algorithm to translate the digitized marker points into positional coordinates in a three dimensional space. The Filter modules will then be used to apply a cubic spline filter onto the data set to remove minor discrepancies during the digitization. Finally the Display modules will generate the desired data results which include the joint angle of the right leg and right foot about the ankle along the $\mathrm{Z}$-axis for each digitized frame within the video file. The results are 
displayed in a tabular or graphical output that can be linked frame-for-frame to the video file. This kinematic data processing is performed for each of the 17 video files recorded for the experiment.

The raw positional data from the APAS software is exported to a spreadsheet file where the information can be further processed using the MS Excel Spreadsheet application. The data associated with the single gait cycle is determines from the video time frame. The start of the gait cycle (i.e. initial contact of the heel) has been synchronized to a timeframe of 0.00 seconds. The end of the gait cycle occurs at the following heel strike of the same leg. The time frame at which this occurs on the video file is used as the end point for the data set to draw up the complete gait cycle. Graphs for the full data set as well as the single gait cycle are generated to provide a visual representation of the joint ankle of the ankle foot complex for each gait scenario.

Because the gait cycles occur at different speeds, comparing the results between gait scenarios based on the time frame results in a misalignment of the gait cycle. Each data point must then be modified to represent the percentage of the gait cycle at which it occurred rather than the time. Once the data has been refined, the resulting performance curves can be plotted based on a $100 \%$ gait cycle. 


\section{RESULTS}

\subsection{Joint Angle Results}

The results of the angular position with respect to time of the right leg for the entire walking session are graphed for each of the five gait scenarios (See Appendix F for full page graphs). Figure 6.1 and 6.2 illustrates the walking patterns exhibiting by the user under the two barefoot walking scenarios (i.e. normal walking and simulated dropfoot).

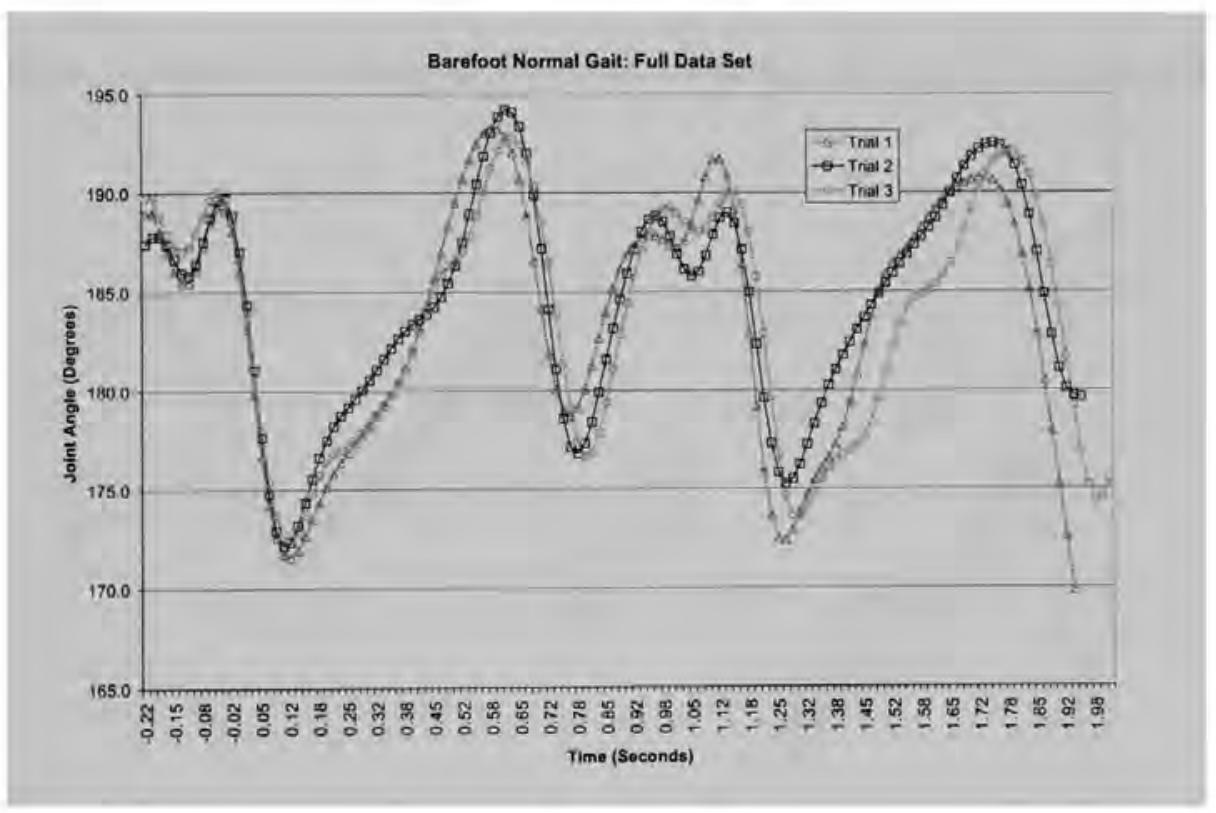

Figure 6.1 Joint angle of right leg for normal barefoot walking (full data set)

Figures $6.3,6.4$, and 6.5 displays the entire walking pattern of the user for each of the three gait scenarios while using the BARA (i.e. normal walking, simulated drop-foot, and BARA assisted movement). 


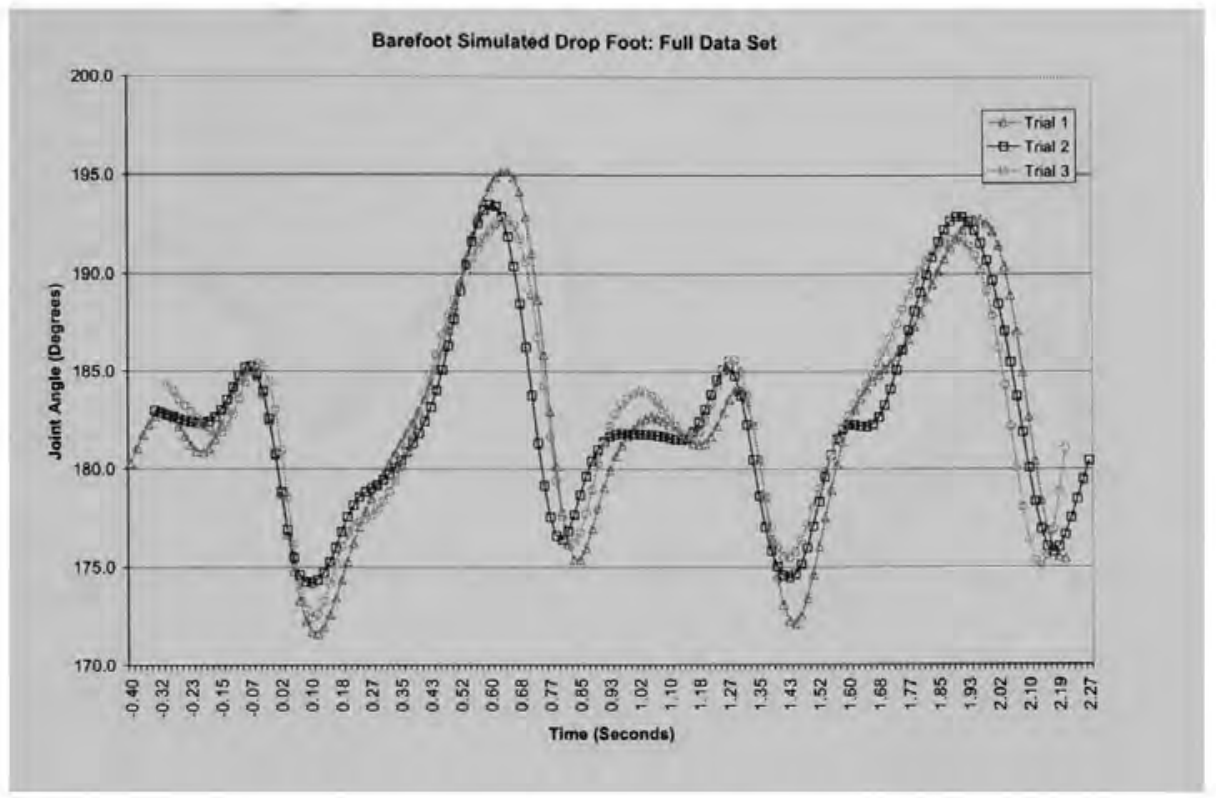

Figure 6.2 Joint angle of right leg for simulated drop-foot barefoot walking (full data set)

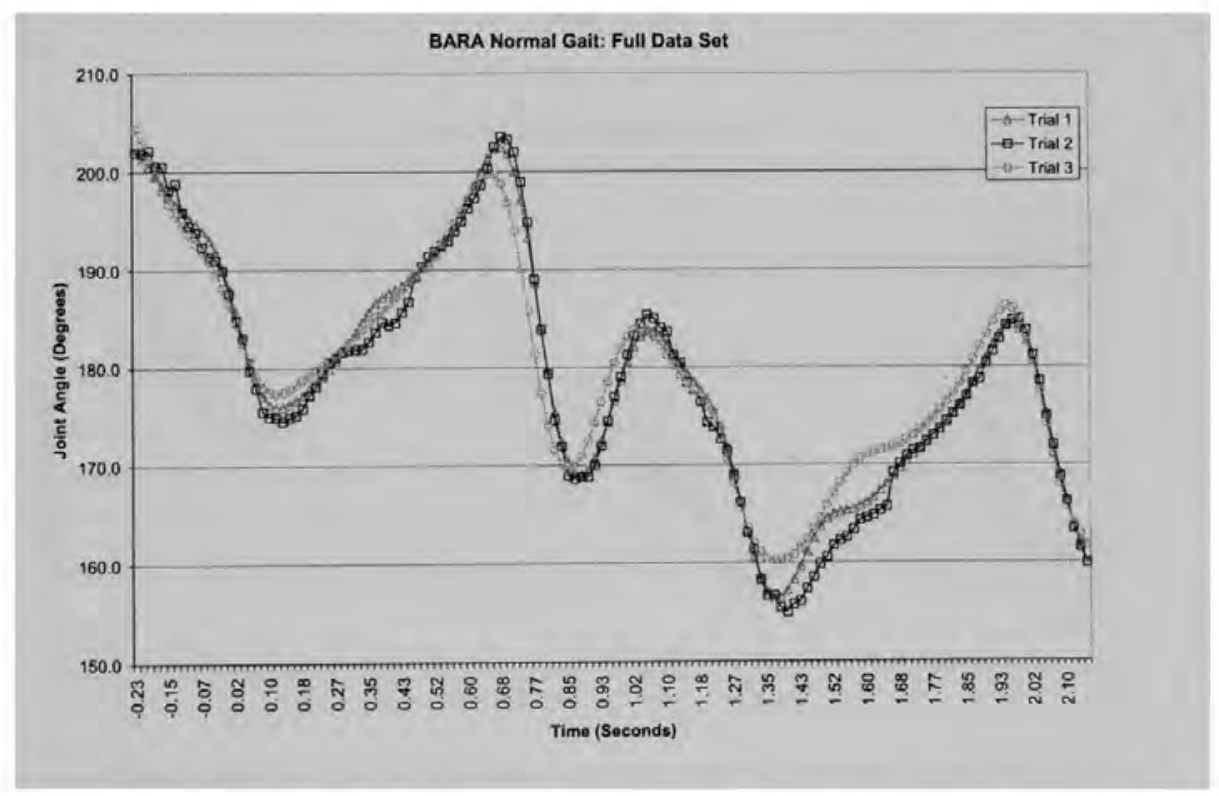

Figure 6.3 Joint angle of right leg for normal walking with BARA powered-off (full data set) 


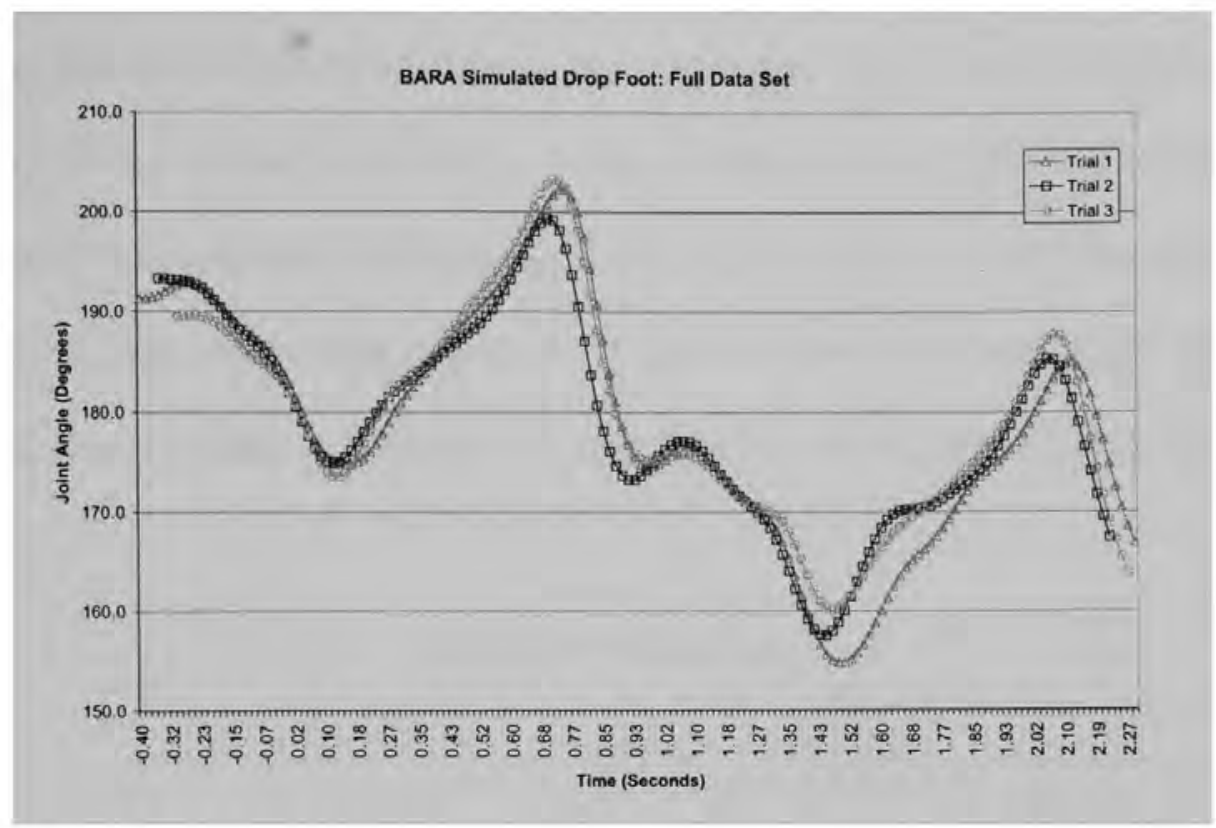

Figure 6.4 Joint angle of right leg for simulated drop-foot walking with BARA powered-off (full data set)

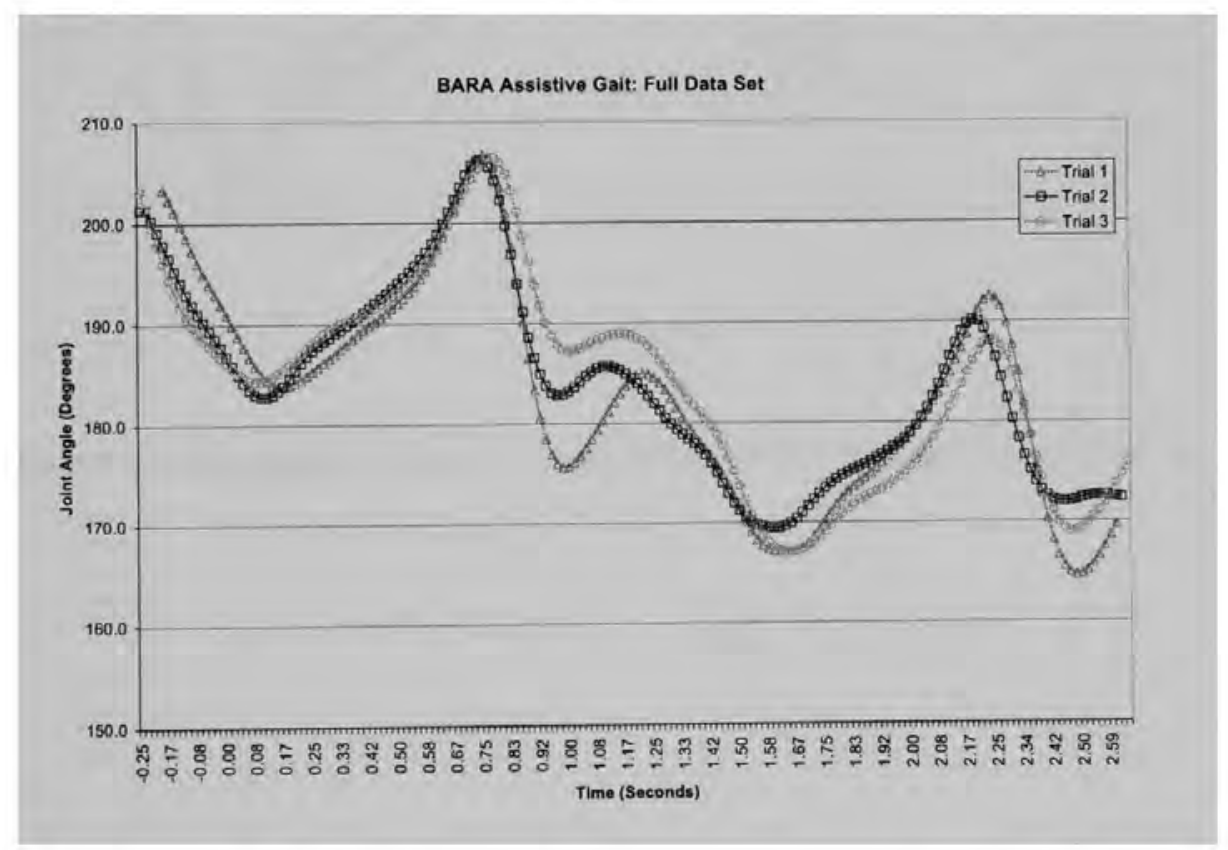

Figure 6.5 Joint angle of right leg for simulated drop-foot walking while using BARA assisted movements (full data set) 
The next set of graphs focuses on the single gait cycle captured within the gait cycle frame of the walking sessions. By using the onset of the heel strikes of the right foot to signify the start and end of the gait cycle, Figure 6.6 thru 6.10 illustrates only the patterns displayed in that single gait cycle. Figure 6.6 shows the joint angle results for normal barefoot walking and Figure 6.7 demonstrates the simulated drop-foot while barefoot.

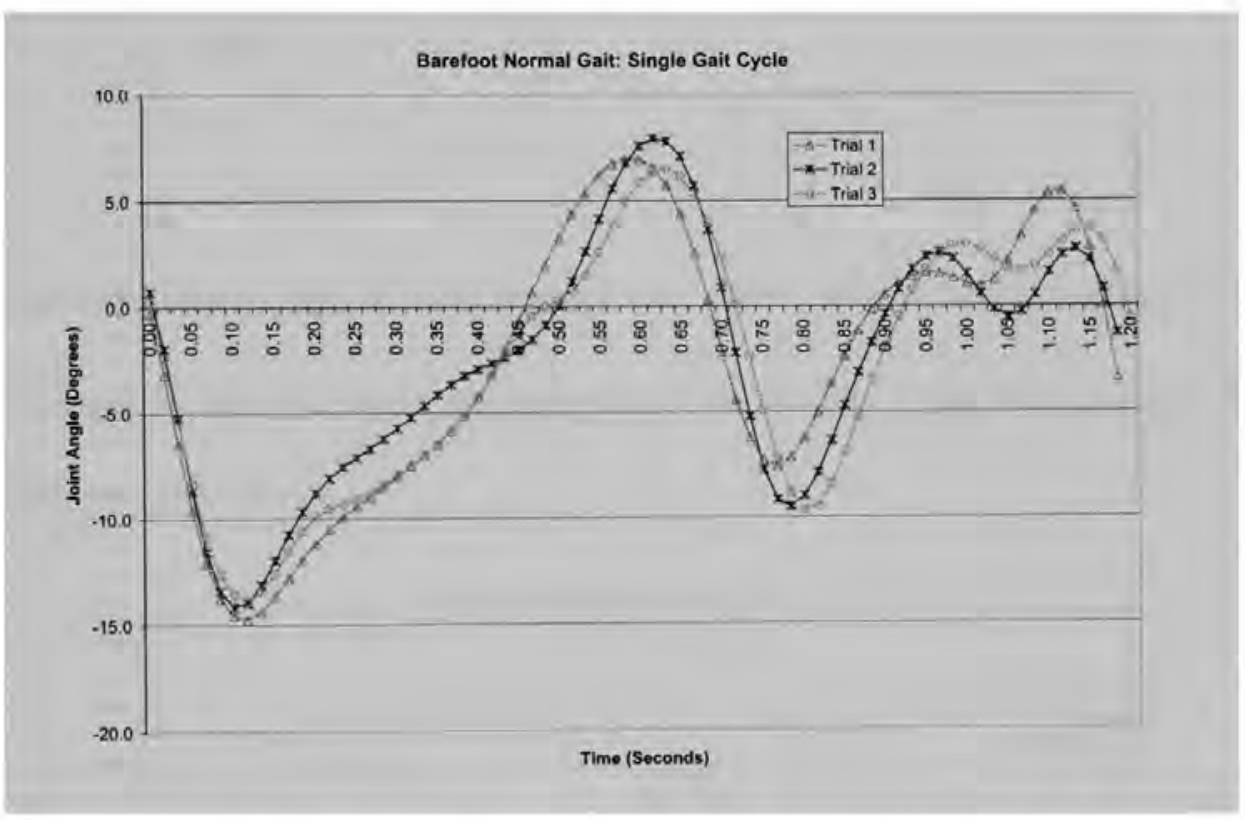

Figure 6.6 Joint angle of right leg for normal barefoot walking (single gait cycle) 


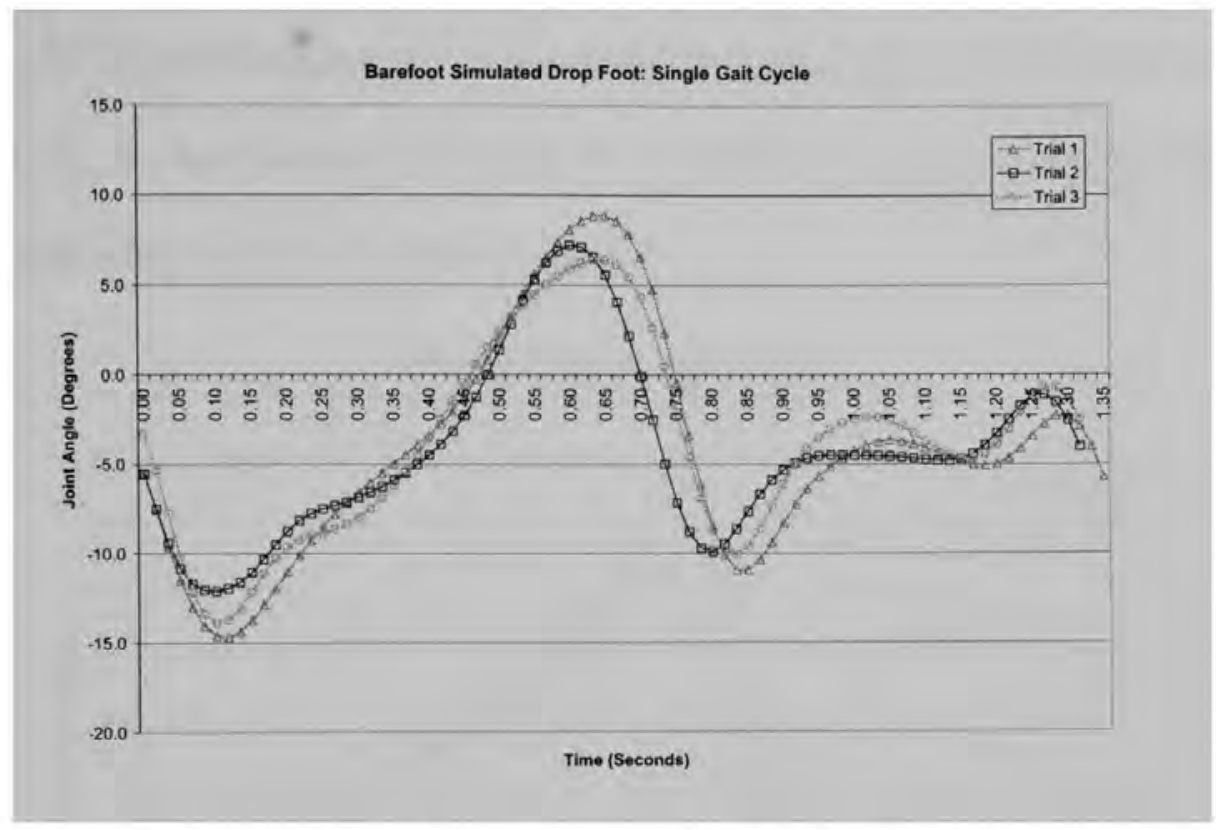

Figure 6.7 Joint angle of right leg for simulated drop-foot barefoot walking (single gait cycle)

Figure 6.8 thru 6.10 also illustrates the joint angle for the gait cycle during which the user is wearing the BARA device.

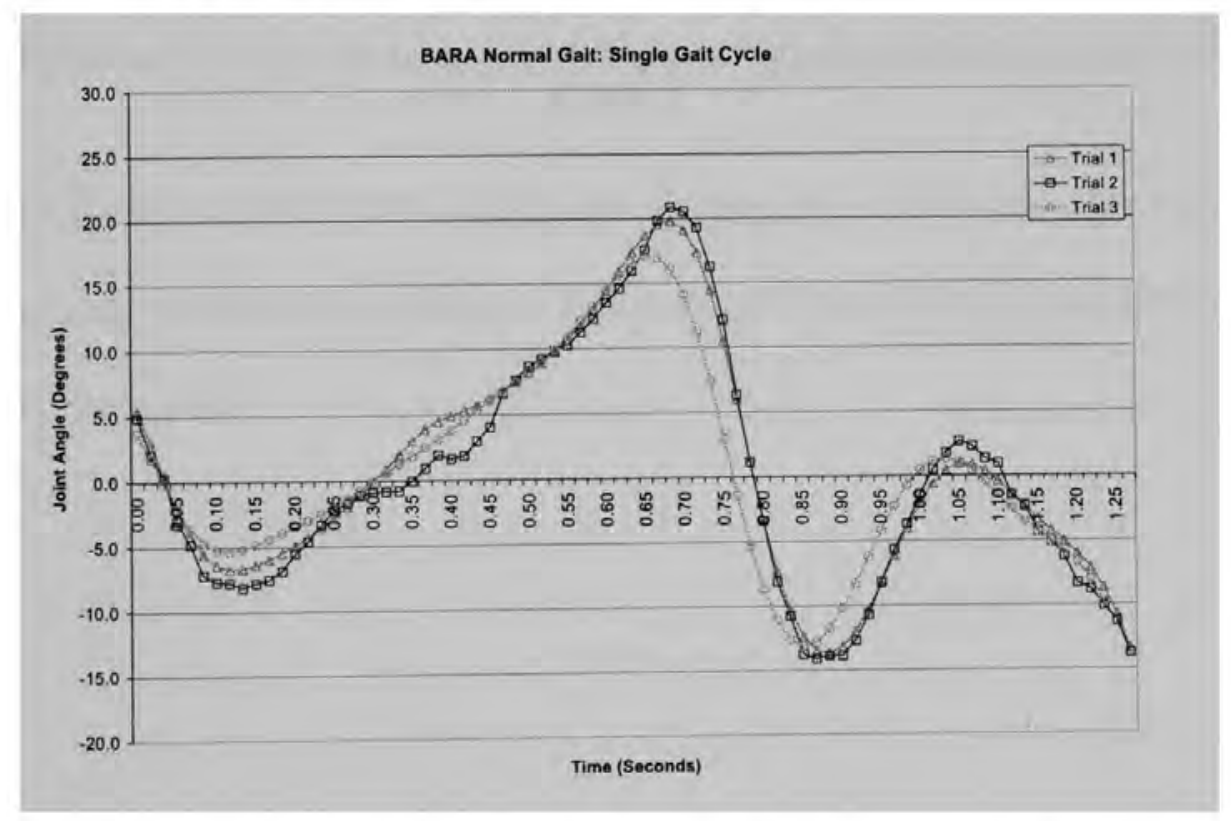

Figure 6.8 Joint angle of right leg for normal walking with BARA powered-off (single gait cycle) 
The results in Figure 6.8 shows the user walking normally with the BARA device turned off. Similarly, the data portrayed in Figure 6.9 represents the user simulating a drop-foot condition also while the BARA device is inactive.

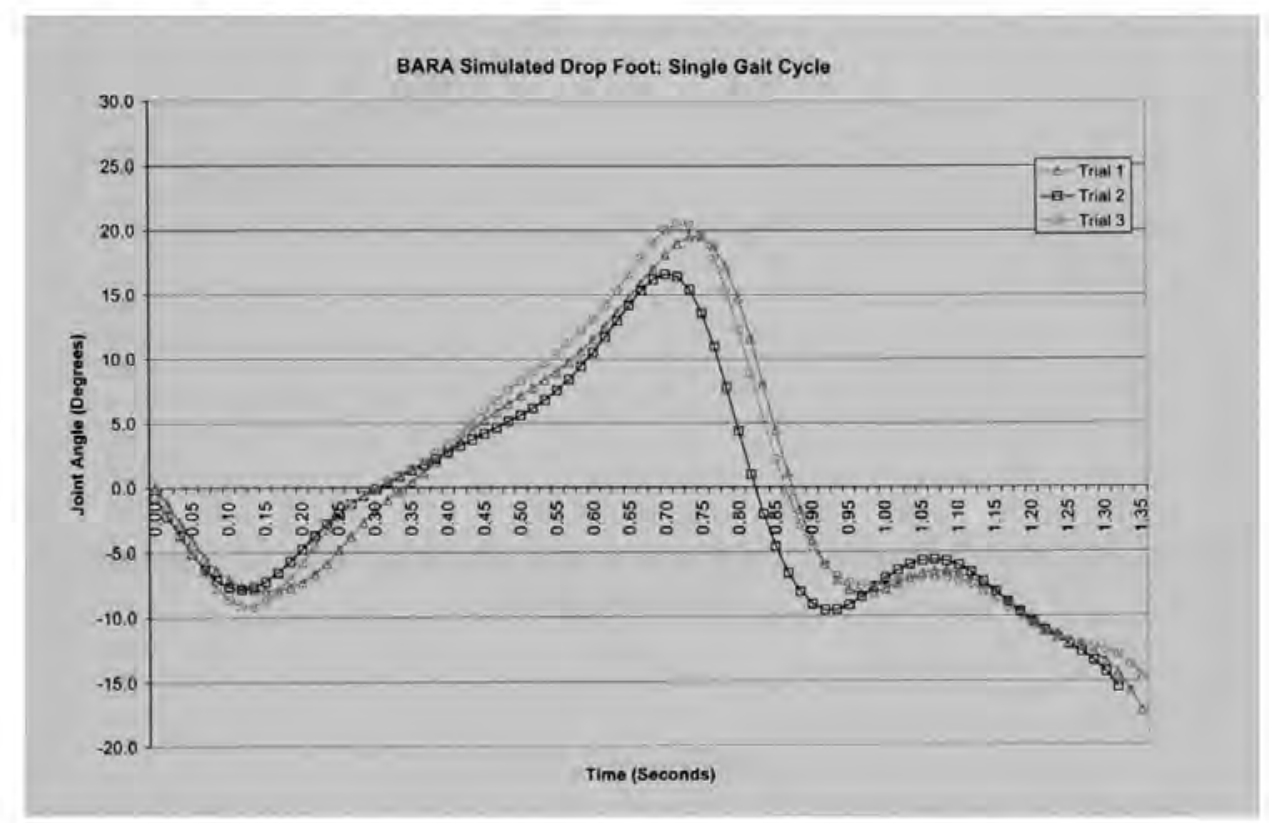

Figure 6.9 Joint angle of right leg for simulated drop-foot walking with BARA powered-off (single gait cycle)

Figure 6.10 shows the final joint angle graph for the single gait cycle where the user is again simulating the drop-foot condition. However, the BARA device has been turned on and properly activated. 


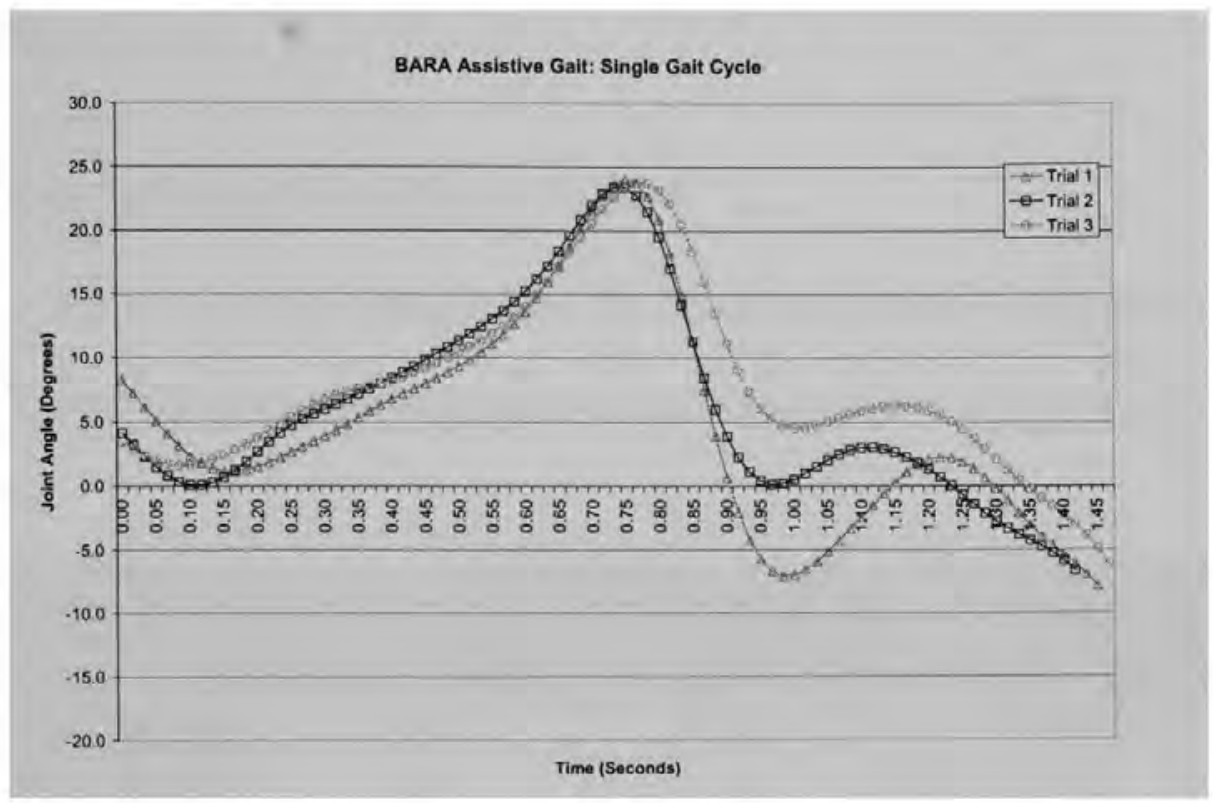

Figure 6.10 Joint angle of right leg for simulated drop-foot walking while using BARA activated (single gait cycle)

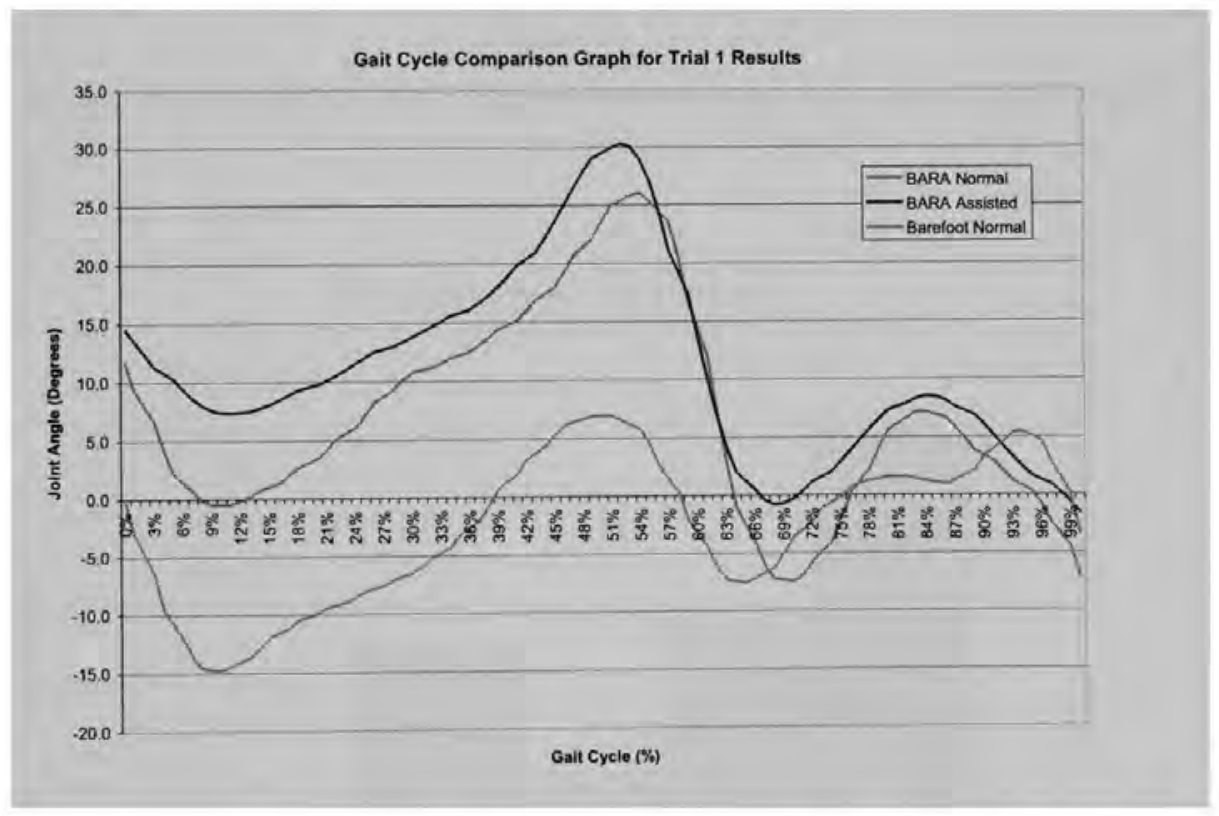

Figure 6.11 Cumulative joint angle curves results of Trial 1 for all the gait scenarios

Figure 6.11 below displays the single gait cycle joint angle curves of Trial 1 for three gait scenarios (i.e. Barefoot normal, BARA normal, BARA assisted). The curves have been normalized from a time dependent $\mathrm{x}$-axis variable to a percentage of gait cycle variable. 


\subsection{Stride Length and Gait Velocity Results}

Table 6.1 provides the average values for the time required to complete a single gait cycle, the stride length, and the walking speed of the right leg for each of the five gait scenarios.

\begin{tabular}{|l|c|c|c|}
\hline \multicolumn{1}{|c|}{ Gait Scenario } & $\begin{array}{c}\text { Avg. Time for } \\
\text { Gait Cycle } \\
\text { (seconds) }\end{array}$ & $\begin{array}{c}\text { Avg. Stride } \\
\text { Length } \\
\text { (cm) }\end{array}$ & $\begin{array}{c}\text { Avg. Walking } \\
\text { speed } \\
\text { (cm/sec) }\end{array}$ \\
\hline Barefoot Normal & 1.19 & 151.8 & 127.6 \\
\hline Barefoot Drop-Foot & 1.33 & 138.8 & 104.5 \\
\hline BARA Normal & 1.26 & 154.4 & 122.9 \\
\hline BARA Drop-Foot & 1.34 & 140.3 & 104.8 \\
\hline BARA Assisted & 1.45 & 141.6 & 97.9 \\
\hline
\end{tabular}

Table 6.1 Average values for duration of gait cycle, stride length, and walking Speed for gait scenarios

Figure 6.12 shows the graphical illustration of the average gait cycle time for each of the gait scenarios.

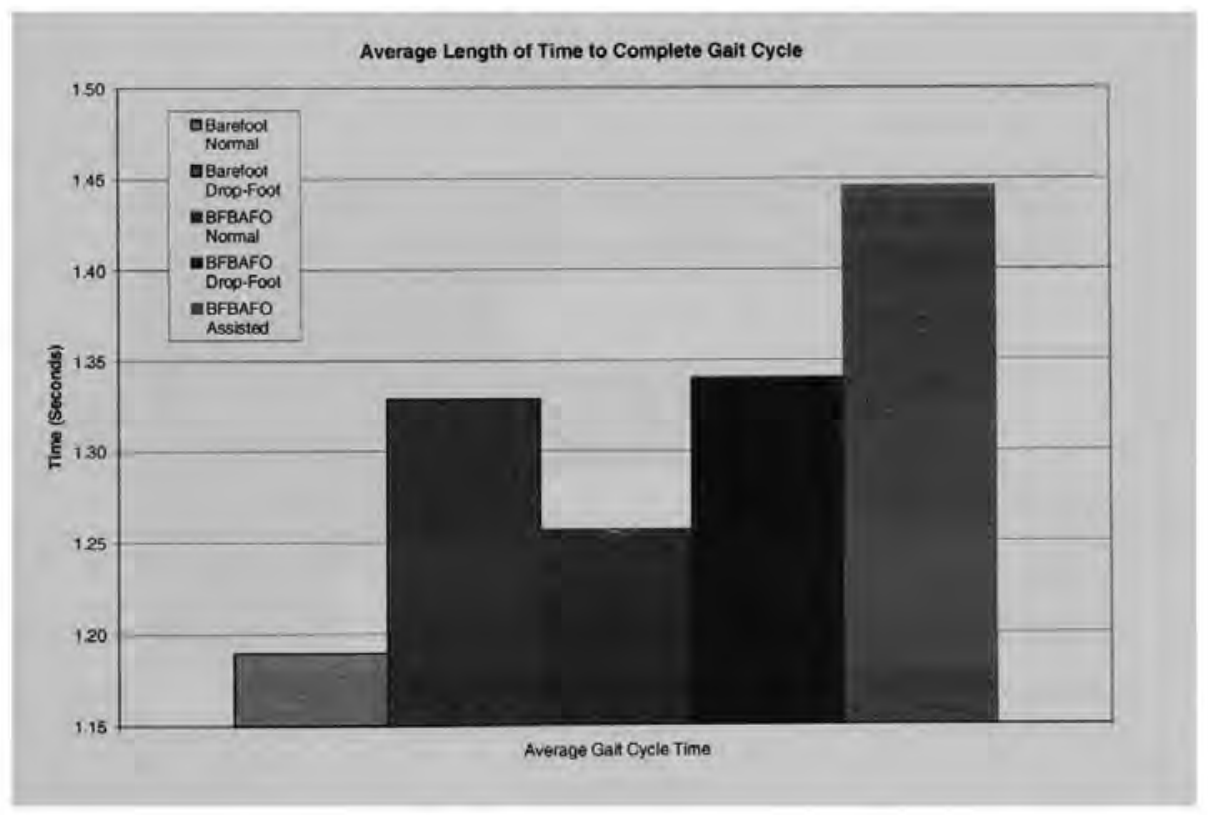

Figure 6.12 Average time to complete a single gait cycle 
Figure 6.13 shows the average stride length in centimeters of the user's right leg during the single gait cycle for each of the five gait scenarios.

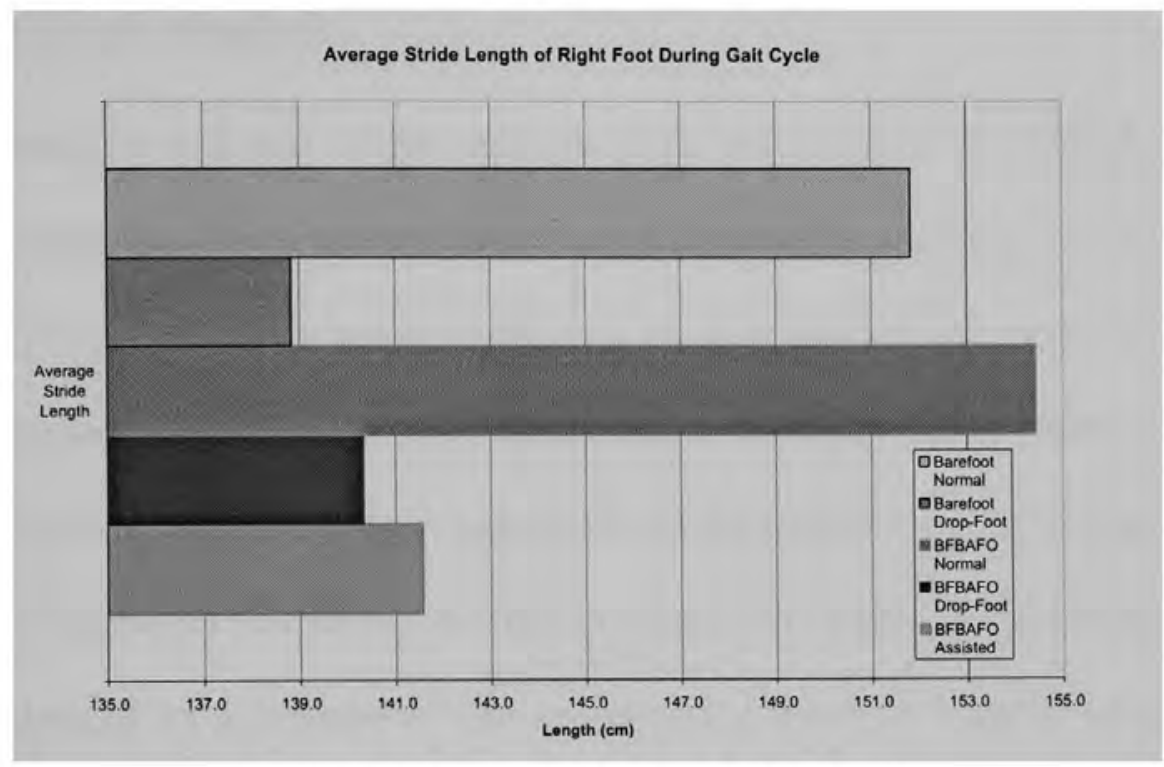

Figure 6.13 Average stride length of gait cycle

Figure 6.14 illustrates the average gait speed achieved by the user during the gait cycle for all five gait scenarios.

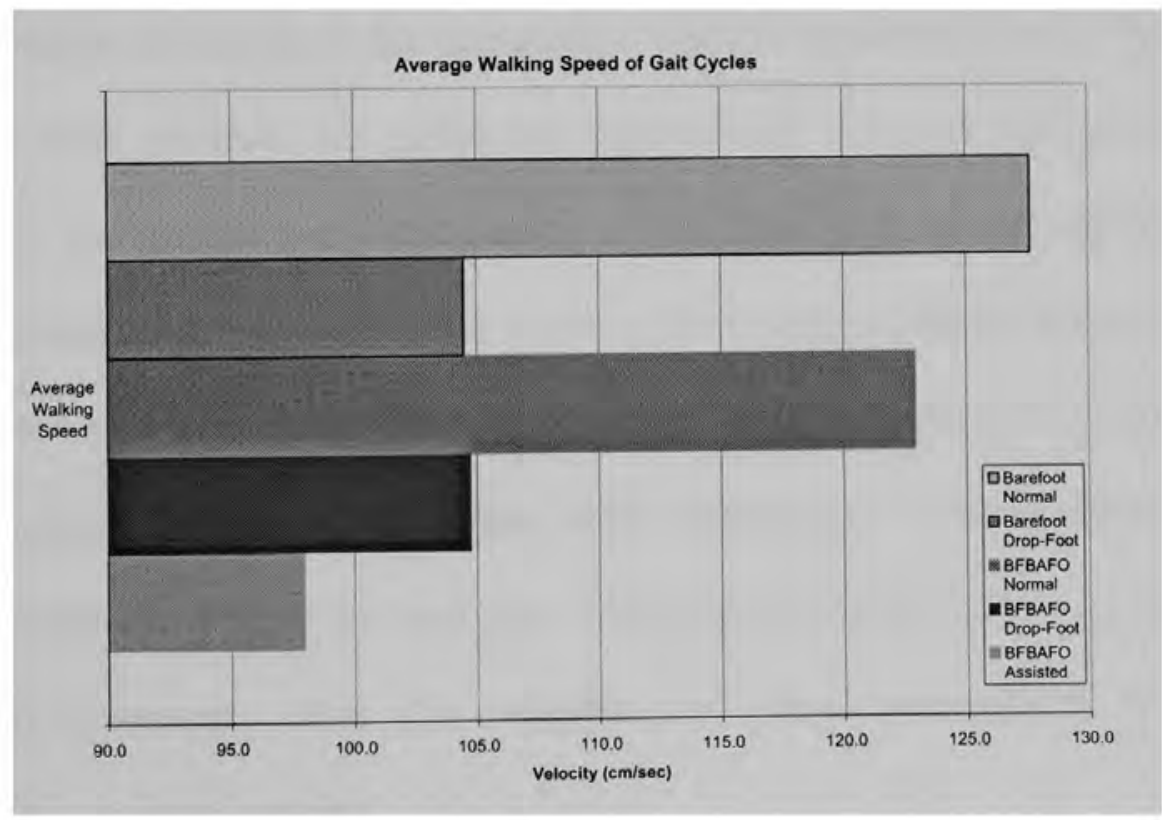

Figure 6.14 Average gait velocity 


\section{DISCUSSION}

\subsection{Joint Angle Analysis}

Because the purpose of this research is to determine if the BARA device is capable of improving gait symmetry in stroke patients with drop-foot into achieving a gait pattern more similar to a healthy individual, the first step was to provide a referential source of data that can be used to objectively compare with the BARA device test results. It was necessary to utilize a subject capable of demonstrating both natural gait and the simulation of drop-foot conditions. It is also necessary to validate the functionality of the testing equipment to determine if the experimental setup is capable of generating acceptable results.

The first step was to validate the experimental setup and evaluate the overall characteristics of the user. Through visual analysis of Figure 6.1, we can clearly see a repetitive pattern of motion in the gait results. This is characteristic of a healthy gait where the basic motions are performed repetitiously with minimal deviations in parameters. The general peaks and valleys of the curve follow what appears to be a horizontal progression. Figure 6.2 also confirms the repetitive pattern of the overall gait analysis. However, because the curve is simulating a handicapped gait cycle (i.e. dropfoot), we notice a deviation in the height of the middle peak. This is reflective of the drop-foot condition where the user has a decreased/inability ability to generate a dorsiflexion movement. This also validates the software's capability in detecting variations in movement patterns. 
The next step was to evaluate the general performance of the BARA device. Figures 6.3 attempts to replicate the normal gait patterns of the same user while wearing the BARA brace. We can determine that there is a similar repetitive pattern exhibited in the joint angle of the ankle-foot complex as compared to the barefoot normal gait curves. Similarly Figure 6.4 shows a close approximation in the general pattern of the barefoot simulation curve for drop-foot condition with the reduction in amplitude of the middle peak. The pattern displayed in Figure 6.5 for the BARA device activated demonstrates the same general repetitive patterns.

The main discrepancy in the results of all the curves in Figures 6.3 thru 6.5 is that the progression of the gait pattern does not follow the same horizontal movement as achieved during the barefoot gait scenarios. There seems to be a large disconnect between the joint angles towards the left of the curve with the joint angles at the right of the curve within the repetitive gait pattern, a difference of approximately $15^{\circ}-20^{\circ}$. The results from the data seems to signify a disjointed gait pattern, however the recording was performed by the same user on a stable and flat surface through a single continuous motion. The leading reason as to the explanation for this discontinuity is based on the setup of the experiment and the existence of a parallax condition. Parallax is defined as an apparent displacement of an object as seen from two different points not on a straight line with the object. Because the investigation is focused on the 2-dimensional movement characteristic of the ankle, it was thought that the use of a single video camera perpendicular to the users walking motion can be used to capture an accurate 2-D representation. It was initially thought that the effects of parallax were negligible. However, due to the design of the BARA and the placement of the ankle and foot marker 
on the brace, there exists a difference in depth of approximately 2 inches between the two markers. Therefore the markers no longer exist in a 2-D plane. Due to this condition, a linear upright position on the left frame of the video is expected to cause a preexisting dorsiflexion. And the same upright position on the right of the video frame will result in a preexisting plantar flexion (Figure 7.1).

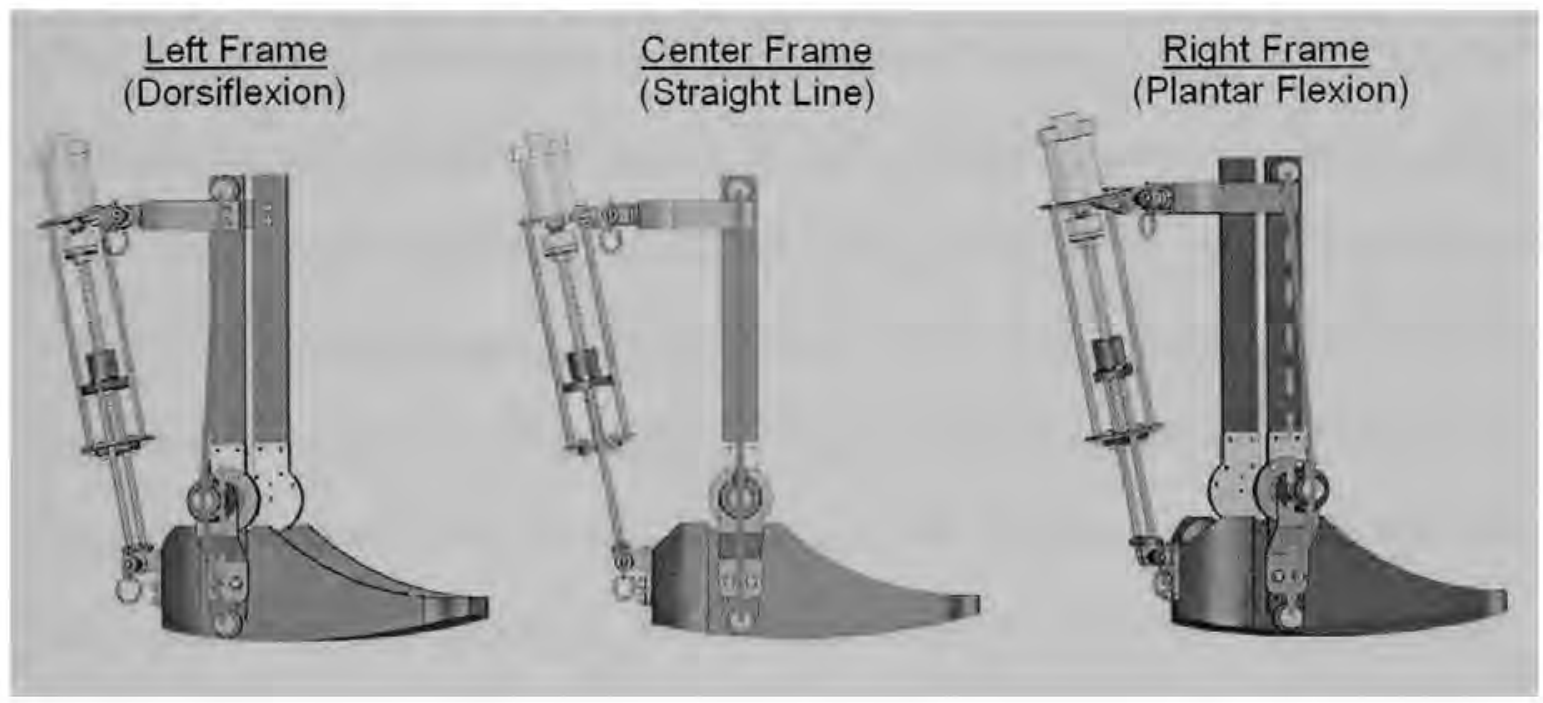

Figure7.1 Effects of Parallax on digitization of BARA video images

The preexisting conditions will gradually minimize as the subject approaches the direct center of the video frame where the difference in depth will not be identifiable by the camera. Although in the barefoot scenarios where the placement of the marker are on the ankle bone and base of the foot, the difference in depth is less than 0.5 in.. The effects of parallax on this setup may be more negligible and therefore unnoticeable from the data acquired. Therefore the general progression of all the BARA data curves seems to follow a downward sloping center line. 
The next step was to focus on a single gait cycle for the different gait scenarios. For the single gait cycle data sets, the pre-calculated neutral standing position (i.e. approximately $180^{\circ}$ ) was subtracted form the raw data to provide the ankle joint movement relative to the neutral position of the user. Figure 6.6 shows in more detail the barefoot normal gait cycle which contains the four primary arcs of motion representative of (1) the initial plantar flexion during the Loading Response, followed by (2) the continuous dorsiflexion past the neutral through Terminal Stance, then (3) the rapid plantar flexion, again past neutral, during Pre-Swing, and finally (4) the last dorsiflexion to the neutral position through the Swing phase to achieve proper foot clearance to avoid dragging of the toe and foot on the ground. The simulated drop-foot results in Figure 6.6 shows the characteristic plantar flexion position at the beginning and end of this gait cycle caused by the users inability to voluntarily raise the foot to a neutral position to achieve toe clearance and proper position for the next heel strike.

A closer inspection of the gait cycle for the BARA gait pattern in Figures 6.8 and 6.9 definitively shows the existence of a dorsiflexion to a near neutral position during swing phase (time $=\sim 1.00 \mathrm{sec}$ ) for normal walking and a lack of significant dorsiflexion for the simulated paralysis sequences (time $=\sim 1.05 \mathrm{sec}$ ). This signifies that the BARA brace does not alters the users gait patterns beyond an acceptable degree. Analyzing the performance with the BARA device activated in Figure 6.10, we see a variation in results during the swing phase. In Trial 1 , there is a noticeable dorsiflexion initiated to slightly pass the neutral position (time $=\sim 1.13 \mathrm{sec}$ ), similar to the desired normal gait pattern. However, trials $2 \& 3$ show a smaller range of movement more similar to that exhibited under drop-foot conditions. However, all three trials show the position of the ankle in a 
relative dorsiflexion position (i.e. above neutral line) during swing phase which is more suitable for toe clearance.

In order to visualize how the gait cycles for the different scenarios compare sideby-side, the results for Trial 1 was normalized to a $100 \%$ gait cycle as shown in Figure 6.11. We can see that the phases and timing at which the ankle motion transition from plantar flexion to dorsiflexion and vice versa for the two gait patterns of the BARA device resembles that of the normal gait throughout the progression of the gait phases. The primary difference is the amplitude of the motion achieved while using the BARA device.

\subsection{Stride Length and Gait Velocity Analysis}

From Table 6.2, the subject in this experiment had an average normal gait cycle of 1.19 seconds. The simulated barefoot drop-foot gait had an average gait cycle of $1.33 \mathrm{sec}$ which is $11.8 \%$ slower. Our user was able to achieve a normal gait cycle while wearing the BARA of $1.26 \mathrm{sec}$. The BARA simulated drop foot had a gait cycle of $1.34 \mathrm{sec}$ which is $6.3 \%$ slower than the normal walking pattern using the BARA device. The gait cycle for the Assisted BARA turned out to be the slowest time at $1.45 \mathrm{sec}$ which is a $15.1 \%$ slower gait cycle than the BARA normal gait. However, the difference in time is slightly attributed to the stride length of the right leg. While simulating the drop-foot conditions barefoot, the user achieved a stride length of $138.8 \mathrm{~cm}$ (a $-8.6 \%$ decrease compared to normal walking). However, with the BARA assisting the user under dropfoot condition, the user was able to display an increase in stride length of $141.6 \mathrm{~cm}$. this is a decrease of $8.3 \%$ compared to normal BARA walking (i.e. $154.4 \mathrm{~cm}$ ). The overall 
walking velocity resulted in the Assisted BARA having the slowest speed of $97.9 \mathrm{~cm} / \mathrm{sec}$ compared to both the barefoot drop-foot $(104.5 \mathrm{~cm} / \mathrm{sec})$ and the BARA simulated dropfoot $(104.7 \mathrm{~cm} / \mathrm{sec})$. The BARA drop-foot had a decrease of $20.4 \%$ compared to normal BARA walking. This is somewhat similar to the $18.2 \%$ decrease in walking speed of the barefoot walking compared to the normal barefoot walking. 


\section{SUMMARY and CONCLUSION}

With the residual physical effects of stroke leading to the \#1 cause of long term disability in the United States, there is a large need to continually seek new technology to help better treat these residual handicaps. Paralysis of the dorsiflexor muscles in the lower leg commonly results in the condition of drop-foot. This form of handicap greatly limits the mobility of the patient and increases the likelihood of falls and secondary injury. There have been several different approaches in developing new rehabilitation treatments focused not only on correcting the superficial problems, but treating the underlying cause of the problem. Programs such as treadmill training, robotic assisted motion, and biofeedback each individually provide aspects that have shown to improve the patient's voluntary muscle control. The aim of this research project was to combine the best characteristics of these techniques into a single robust, low-cost, portable prototype unit that can be used in a rehabilitation setting to improve voluntary muscle control and gait symmetry in stoke patients.

The overall construction of the BARA device is comprised of basic commercially available components utilizing minimal customized third party items which typically increase production costs. The brace design allows for general use by multiple patients because it does not require any custom fitting besides a matching shoe size. The device has an approximate total weight of $8.8 \mathrm{lbs}$ and a overall dimension of approximately 17 in. $x$ 17in. $x$ 8in.. The mechanical features are constructed using metal components to ensure high durability, low maintenance, and a long life which are beneficial in a rehabilitation center. 
The control system is also designed to allow portability for use with the BARA device. The entire system can be powered using any standard outlet providing a near endless continuous usage of the device. The control system has a simple user interface including just two toggle switches (i.e. power and mode selection) and two turn knobs for customizing the sensitivity of the load cell to the user. No additional equipment is required (e.g. computer) to properly setup the device for use because the control system includes LED displays which provide visual status display to the operation of the BARA device.

The BARA can be used in two different rehabilitation modes, both centered on characteristics which have shown to be effective in treatment. The Dorsiflexion Training mode utilizes electromyography biofeedback to help supplement the lost sensory perception of the user. This promotes focused and active participation on the part of the patient. The reinforcement is provided in the form of a visual and/or audio feedback in addition to the physical movement of the joint by the robotic component which simulates the desired movement. The motion is triggered by the EMG biofeedback device when the users EMG signal reaches an adjustable threshold level. This allows the device to continually challenge the user's ability as they improve. This exercise can be performed in a sitting position without the participation of a physical therapist. The Gait Training mode provides a tool designed to be used in a walking exercise program. This task specific exercise promotes gait re-education by allowing the user to perform the actual motion of walking while correcting their drop-foot condition. This is accomplished either through their own manual attempt incorporating the EMG biofeedback or automatically via the robotic component as a secondary safety measure. The device will 
always insure the foot will rapidly move to a neutral position during the swing phase to prevent the toes from dragging the floor which commonly result in a loss of balance.

The experimental procedure was used to evaluate the performance characteristic of the prototype BARA in a walking situation. Although the joint angle data curves about the right ankle exhibited similar overall patterns as that produced by normal walking, the progression of the gait cycle through multiple gait cycles exhibited a degrading yet consistent joint angle pattern. This result is thought to be caused by the effects of parallax due to the use of only one video camera and on the placement of the limb markers which do not rest in a 2-dimensional plane. The extent of the angular discrepancy caused by this effect is unknown at this time. Due to this inaccuracy in the data results, definitive conclusions may not be appropriate based on the given setup and test results. However, the acceptable conclusions can be inferred based on the known effects of parallax for this preliminary pilot study.

Focusing on the results of the individual gait cycles shows that the BARA gaits exhibit the same ankle arcs of motion as normal walking which signifies that the use of the BARA does not alter the users gait to an unacceptable degree. However, the ranges of angular motion are not identical. The key result from these curves show that when the BARA is powered on and providing assisted motion, the ankle does automatically recovers and/or remains in a neutral to slight dorsiflexion position which allows for proper clearance of the toe and foot during the swing phase of gait. This properly corrects the drop-foot condition in the user's gait. Having the BARA assist the user resulted in an overall slower gait cycle and walking velocity, but achieved a slightly longer stride length. This result may be attributed to the fact that the user did not have 
extensive practice with using the BARA. Therefore, even with the BARA device correcting the drop-foot condition, the user was instructed to continue simulating paralysis of the dorsifleixon muscles. This unfamiliarity with the device may result in the user being not yet comfortable enough to attempt normal gait patterns while still exhibiting paralysis of the dorsiflexor muscle. Therefore due to the extra mass of the device resulted in a slower gait cycle and slower velocity than expected.

In conclusion, the BARA device provides an acceptable first prototype design which can perform the basic responsibilities that it was designed to do. The BARA device successfully integrates an EMG biofeedback signals into a portable assistive AFO that can be used for gait specific training. The device can serve as a versatile and portable tool for use in a rehabilitation setting. The controls and interface require very basic training in order to operate. We can infer with a high degree of confidence that plantar flexion as a result of drop-foot during swing phase is corrected which signifies that the BARA device allows the user to achieve a similar ankle movement patterns as normal walking. By integrating the biofeedback training along with the robotic assisted motion and task specific exercises, it is believed that this new technology for rehabilitation can provide dramatic improvements in voluntary muscle control to both acute and chronic stroke patients with similar physical handicaps. 


\section{RECOMMENDATION AND FUTUREWORK}

The BARA prototype showed promising results based on the preliminary design and evaluation presented within this report. However, it is understandable that there are several items within the scope of this device that can be addressed to further improve the operation and investigational evaluation of the BARA device.

To address the design consideration, several components within the prototype BARA device were manufactured and assembled based on available resources and preliminary designs. Therefore much of the mechanical components such as the actuator and connectors/mounts of the BARA brace were manufactured from raw material and assembled modularly using basic nuts and bolts. The ability to manufacture several of the components to form a single solid structure would simplify the assembly and structural integrity of the device further. Utilizing lighter weight material could then be considered which would reduce the overall weight of the device and further improve the performance of the BARA.

Further improvements in the overall BARA design may consider the addition of yet another unique and effective rehabilitation technique of Functional Electrical Stimulation (FES). Utilizing a light electrical stimulation may help improve the physiological condition of the muscle by actificially inducing a complete muscle contraction.

With the basic schematic of the control system having been tested and validated to function properly on the modified protoboard, a printed circuit board would be a beneficial option that would further reduce the size of the control system to provide a 
more user acceptable device. A protective outer casing around the circuit board would greatly improve its durability from accidental damage to the delicate circuitry within. A second option would be the repositioning of the control circuit as a stationary device along side the power supply. This would entail running control wires from the control system to the user which would reduce the amount of equipment the user would need to dawn while using the BARA. Additional enhancements may include the usage of wireless communication devices which would provide the user with a higher unimpeded mobility

It was pointed out in the report that the experimental setup utilizing a single video camera to capture the kinematic gait motion of the BARA device and the placement of the reflective markers onto differing planes may be attributed to the unusual data results obtained for the BARA gait test sessions. It is recommended that incorporating a multiple camera setup at different viewing angles to capture the video movement of the reflective markers would allow the APAS software to correct the parallax effects seen in the current experimental single camera setup. This should result in possibly more accurate joint angle data throughout the entire video frame.

With this report being the initial pilot study into the general performance of the BARA prototype, it was acceptable and convenient from a safety perspective to utilize a healthy able bodied test subject. The eventual goal for this research topic would be to include subject from a clinical source. Being able to obtain actual stroke patients exhibiting drop-foot condition to participate in the research study would provide more authentic data results as to the actual influence the BARA device has on improving gait symmetry. 
This initial study focused primarily only on the 2-dimentional movement of the ankle joint during gait. However, a comprehensive analysis on the kinematics of gait would require a 3-dimensional perspective in addition to the inclusion of the full leg, hip, and possible full body analysis. A comparative analysis would need to be performed on the contralateral side of the subject as well to determine what the translating effect may be.

The device was initially designed for stroke rehabilitation. But the concept can be adapted to provide a larger field of usage to include supports for broken legs or post surgical procedures. It may be further developed to provide enhanced performance in a military or commercial setting. 


\section{LIST OF REFERENCES}

American Heart Association, "Heart Disease and Stroke Statistics - 2006 Update", [online], 2005, http://www.americanheart.org/downloadable/heart/1135358648580551026_HS_Stats06book.pdf (Accessed: February 25, 2006)

American Heart Association, "Stroke Risk factors", [online], 2007, www.americanheart.org/presenter.jhtml?identifier=4716 (Accessed: March 6, 2007)

Basmajian JV, Kukulka CG, Narayan MG, and others Source. Biofeedback treatment of foot-drop after stroke compared with standard rehabilitation technique: effects on voluntary control and strength. Archives of physical medicine and rehabilitation. 1975 Jun; 56(6): pp231-236.

Bobath B (1978). Adult hemiplegia: Evaluation and Treatment. London: Heinemann Medical Books.

Barbeau $\mathrm{H}$, Fung $\mathrm{J}$. The role of rehabilitation in the recovery of walking in the neurological population. Current Opinion in Neurology 2001;14: pp734-740

Barbeau H, Rossignol S. Recovery of locomotion after chronic spinalization in adult cat. Brain Res 1987;412: pp84-95

Bobath B. Treatment of adult hemiplegia. Physiotherapy. 1977 Oct; 63(10): pp 310-313.

Bobath B. Adult Hemiplegia: Evaluation and Treatment. London: Heinemann Medical Books. 1978.

Brunnstrom S. Movement Therapy in Hemiplegia: a Neurophysiological Approach. New York: Harper \& Row. 1970

Bütefisch C, Hummelsheim H, Denzler P, Mauritz KH. Repetitive training of isolated movements improves the outcome of motor rehabilitation of the centrally paretic hand . Journal of the neurological sciences. 1995 May; 130(1): pp59-68.

Danaher Motion, "Ball and Lead Screws Catalog - February 2004 Release”, [online], 2004, http://www.danahermotion.com/documents/index.php?product_cat_id=211 (Accessed: September 11, 2006)

Fasoli SE, Krebs HI, Stein J, Frontera WR, Hogan N. Effects of robotic therapy on motor impairment and recovery in chronic stroke. Archives of Physical Medicine and Rehabilitation. April 2003; 84(4): pp477-482 
Hack W, Kaste M, Bogousslavsky J, Brainin M, Chamorro A, Lees K, Leys D et al. European Stroke Initiative Recommendations for Stroke Management-update 2003. Cerebrovasc Disorder. 2003;16: pp311-337

Hesse S, Bertelt C, Jahnke MT, Schaffrin A, Baake P, Malezic M, Mauritz KH. Treadmill training with partial body weight support compared with physiotherapy in nonambulatory hemiparetic patients. Stroke 1995a;26: pp976-981

Hogan N, Krebs HI, Sharon A, Charnnarong J. Interactive robotic therapist. Massachusetts Inst. Technology. Cambridge, U.S. Patent \#5 466213, 1995

Intiso D, Santilli V, Grasso MG, Rossi R, Caruso I. Rehabilitation of walking with electromyographic biofeedback in foot-drop after stroke. Stroke; a journal of cerebral circulation. 1994 Jun; 25(6): pp1 189-92.

Johnstone M. illustrated by Estrid Barton. "Restoration of motor function in the stroke patient : a physiotherapist's approach". Published: Edinburgh ; New York : Churchill Livingstone, 1987 3rd ed.

Kaplan P., Cerullo L, "Stroke Rehabilitation”. Published: Boston : Butterworths, c1986

Karni A. The acquisition of perceptual and motor skills: a memory system in the adult human cortex. Brain research. Cognitive brain research. 1996 Dec; 5(1-2): pp39-48.

Krebs HI, Hogan N, Aisen ML, Volpe BT. Robot-Aided Neuro-Rehabilitation; IEEE Transactions on Rehabilitation Engineering, 1998; 6(1): pp75-87

Lehmann JF, DeLateur BJ, Fowler RS Jr, Warren CG, Arnhold R, Schertzer G, Hurka R, Whitmore JJ, Masock AJ, Chambers KH. Stroke: Does rehabilitation affect outcome? Archives of physical medicine and rehabilitation. Sep 1975; 56(9): pp375-382.

Liepert J, Miltner WH, Bauder H, Sommer M, Dettmers C, Taub E, Weiller C. Motor cortex plasticity during constraint-induced movement therapy in stroke patients. Neuroscience letters. 1998 Jun 26; 250(1): 5-8.

Lovely RG, Gregor RJ, Roy RR, Edgerton VR. Effects of training on the recovery of fullweight-bearing stepping in the adult spinal cat. Exp Neurol 1986; 92: pp 421-435

Martini F, Timmons M, Tallitsch R, "Human Anatomy", $4^{\text {th }}$ ed., New Jersey: Pearson Education, 2003, pp 313-438.

Mayer RF, Young JL. The effects of hemiplegia with spasticity. In Feldman GR, Young RR and Koella WP (eds): Spasticity: Disorder Motor Control, Chicago Year Book Medical Publisher, 1980 
Mauritz KH. Gait training in hemiparetic stroke patients. Eura Medicophys. 2004 Sep;40(3): pp 165-78.

McComas AJ, et al: Functional changes in motorneuron of hemiparetic patients. J. Neurol, Neurosurg, and psychiatry. 1973; 36: pp 183-193

Murray MP, Drought AB, Kory RC. Walking patterns of normal men. J Bone Joint Surg. 1964; 46A(2): pp 335-360

National Institute of Neurological Disorders and Stroke (NINDS), "Stroke: Hope Through Research. National Institutes of Health", [online], 1999, www.ninds.nih.gov/disorders/stroke/detail_stroke.htm (Accessed: March 06, 2007)

Neal B, MacMahon S, Chapman N. Effects of ACE inhibitors, calcium antagonists, and other blood-pressure-lowering drugs: results of prospectively designed overviews of randomizes trials. Blood Pressure Lowering Treatments Trialists' Collaboration. Lancet 2000;356:pp 1905-1964

Nichols DS. Balance retraining after stroke using force plate biofeedback. Phys Ther 1997;77: pp 553-558

Perry J, "Gait Analysis, Normal and Pathological Function", New Jersey: SLACK Incorporated, 1992, pp 469

Robert WT, Sanjit KB, Norine CF, Mark RS. "Gait retraining post stroke". Topics in stroke rehabilitation. 2003 Summer; 10(2): pp 34-65

Rosenfalck A, Andreassen S. Impaired regulation of force and firing pattern of single motor units in patients with spasticity. J. Neurol Neurosurg Psychiatry 1980; 43: pp 907916

Silver KH, Macko RF, Forrester LW, Goldberg AP and Smith GV. Effects of aerobic treadmill training on gait velocity, cadence, and gait symmetry in chronic hemiparetic stroke: a preliminary report. Neurorehabil Neural Repair 2000;14: pp 65-71

Silverthorn D.U., "Human Physiology An Integrated Approach", 3 rd ed., San Francisco: Benjamin Cummings, 2004, pp 293-442.

Singleton M., Branch E., co- editors. "Physical therapy and the stroke patient : pathologic aspects and clinical management". Published: New York : Haworth Press, c1987

Senelick Richard C., Rossi, Peter W., Dougherty, Karla (1994). Living with Stroke: A Guide For Families. Contemporary Books, Chicago. 
Shepherd RB. Exercise and training to optimize functional motor performance in stroke: driving neural reorganization? Neural Plast 2001;8: pp 121-129

Shinton R, Beevers G. Meta-analysis of relation between cigarette smoking and stroke. Bmj 1989; 298: pp 789-794

Staessen JA, Wang JG, Thijs L. Cardiovascular protection and blood pressure reduction: a meta-analysis. Lancet 2001; 358: pp 1305-1315

Stanford Hospital \& Clinics, "Cardiovascular Diseases: Effects of Stroke", [online], 2007, www.stanfordhospital.com/healthLib/atoz/cardiac/effects.html (Accessed: March $7,2007)$

Susan E. Fasoli, Hermano I. Krebs, Joel Stein, Walter R. Frontera and Neville Hogan. Archives of Physical Medicine and Rehabilitation. April 2003; 84(4): pp 477-482

Takebe K, Basmajian JV. Gait analysis in stroke patients to assess treatments of footdrop. Archives of physical medicine and rehabilitation. 1976 Jul; 57(1): pp 305-10.

Tang A, Rymer WZ. Abnormal force-EMG relation in paretic limbs of hemiparetic human subjects. J. Neurol Neurosurg Psychiatry 1981;44: pp 690-698

Trombly CA, Quintana LA. Differences in response to exercise by post CVA and normal subjects. The Occupational Therapy Journal Research. 1985;5: pp 39-58

Titianova EB, Pitkanen K, Paakkonen A, Sivenius J, and Tarkka IM. Gait characteristics and functional ambulation profile in patients with chronic unilateral stroke. Am J Phys Med Rehabil 2003; 82: pp 778-786

Villarosa, Linda, Ed., Singleton, LaFayette, MD, Johnson, Kirk A. (1993). "Black Health Library Guide to Stroke". Henry Holt and Company, New York.

Visintin M, Barbeau H, Korner-Bitensky N, Mayo NE. A new approach to retrain gait in stroke patients through body weight support and treadmill stimulation. Stroke 1998;29: pp 1122-1128

Volpe BT, Krebs HI, Hogan N, Edelstein L, Diels CM, Aisen ML. Robot training enhanced motor outcome in patients with stroke maintained over 3 years. Neurology 1999;53: pp 1874-1876

Westcott EJ. Traditional exercise regimens for the hemiplegic patient. American journal of physical medicine. Am J Phys Med. 1967 Feb; 46(1): pp 1012-23. 
Wolf SL, Baker MP, Kelly JL. EMG biofeedback in stroke: a 1-year follow-up on the effect of patient characteristics. Archives of physical medicine and rehabilitation. 1980 Aug; 61(8): pp 351-5 


\section{APPENDICES}

Appendix A: Engineering Drawing

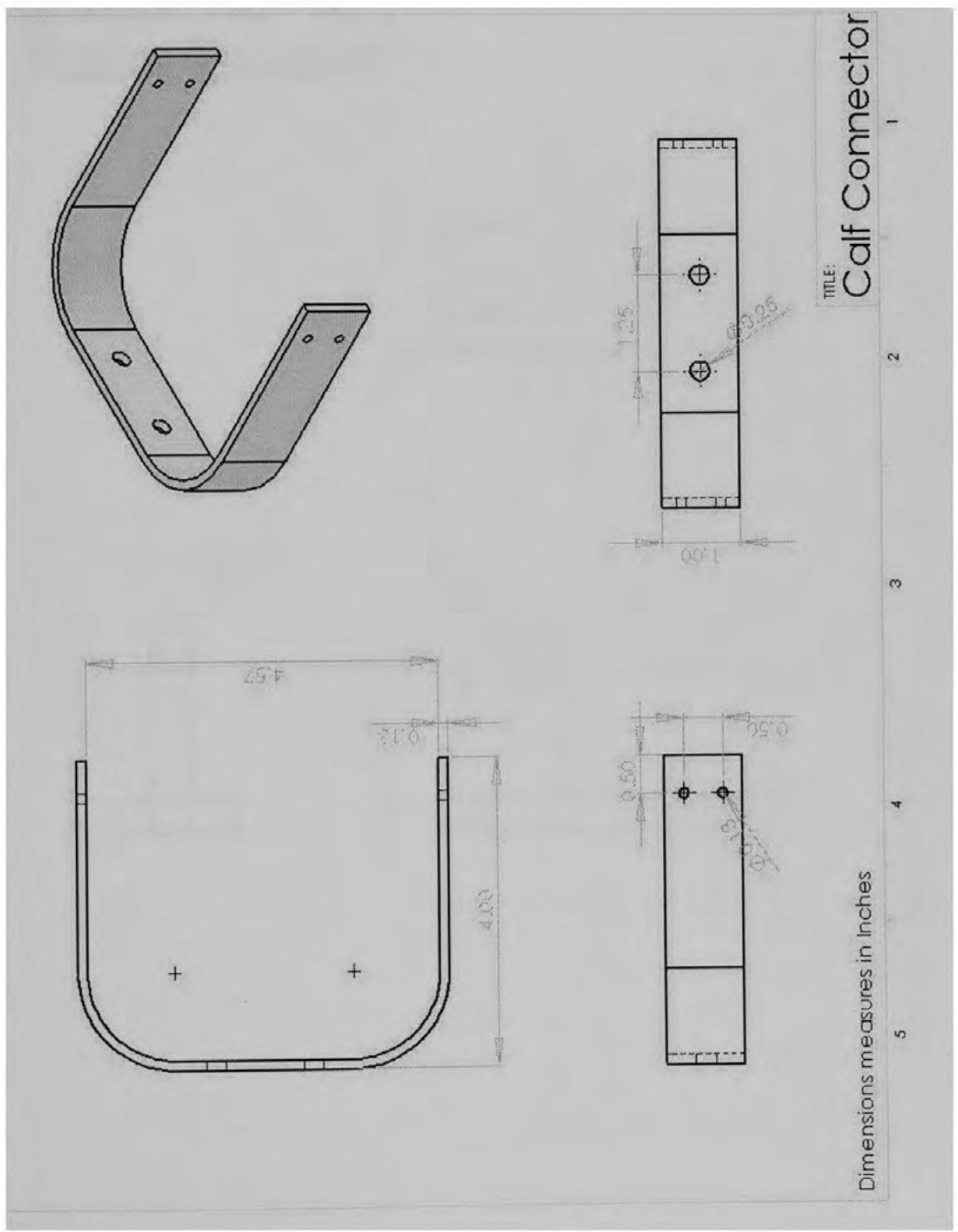



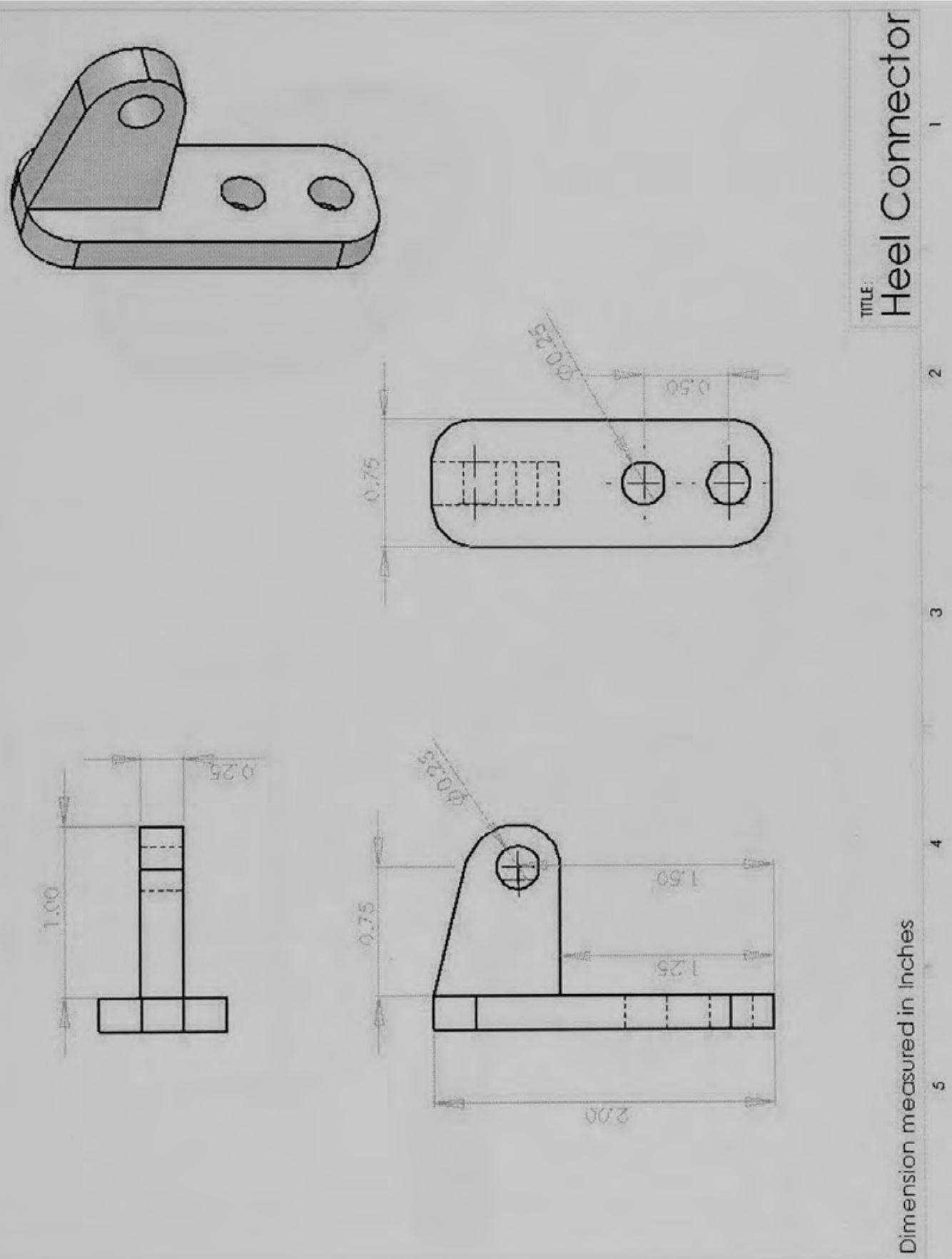

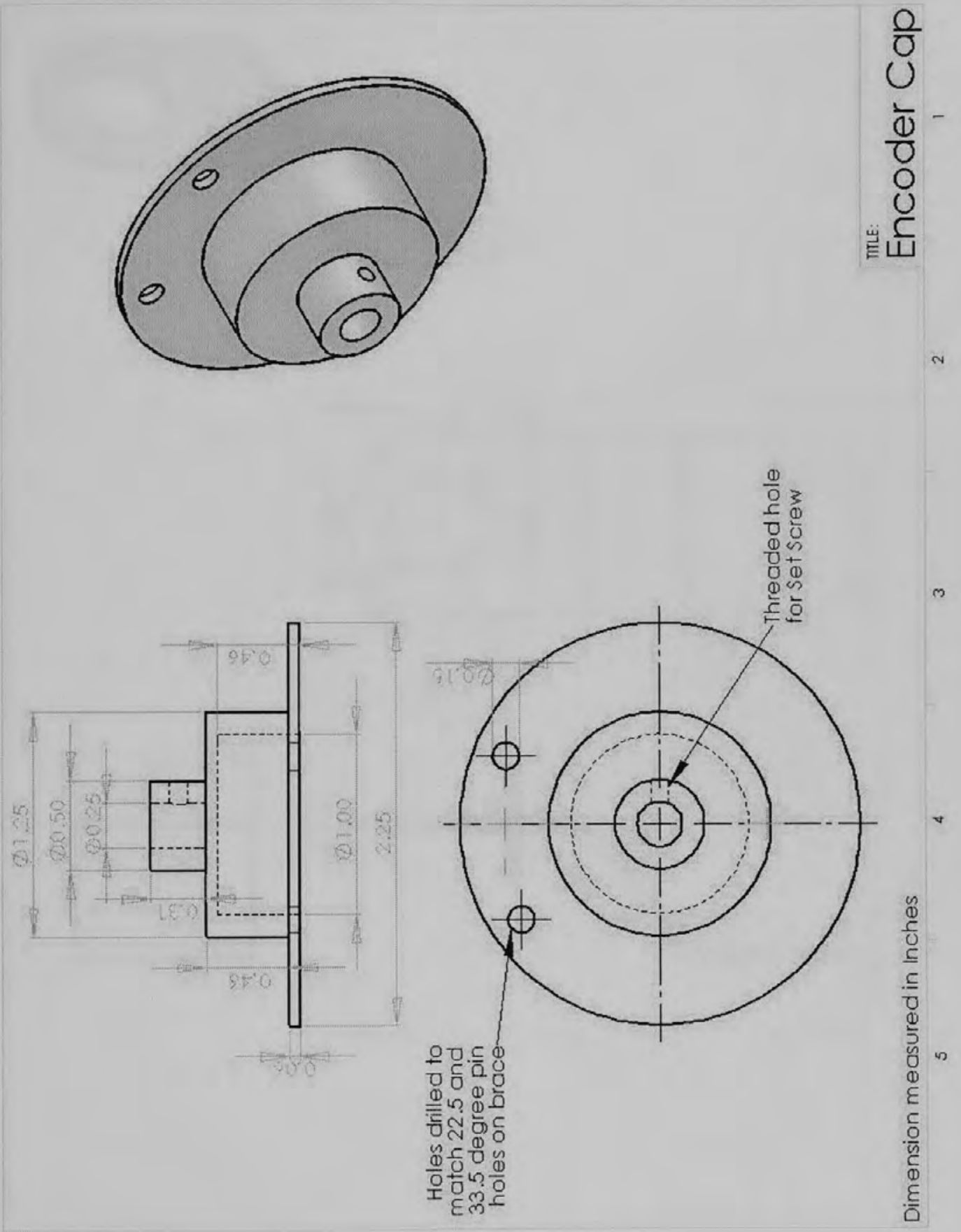

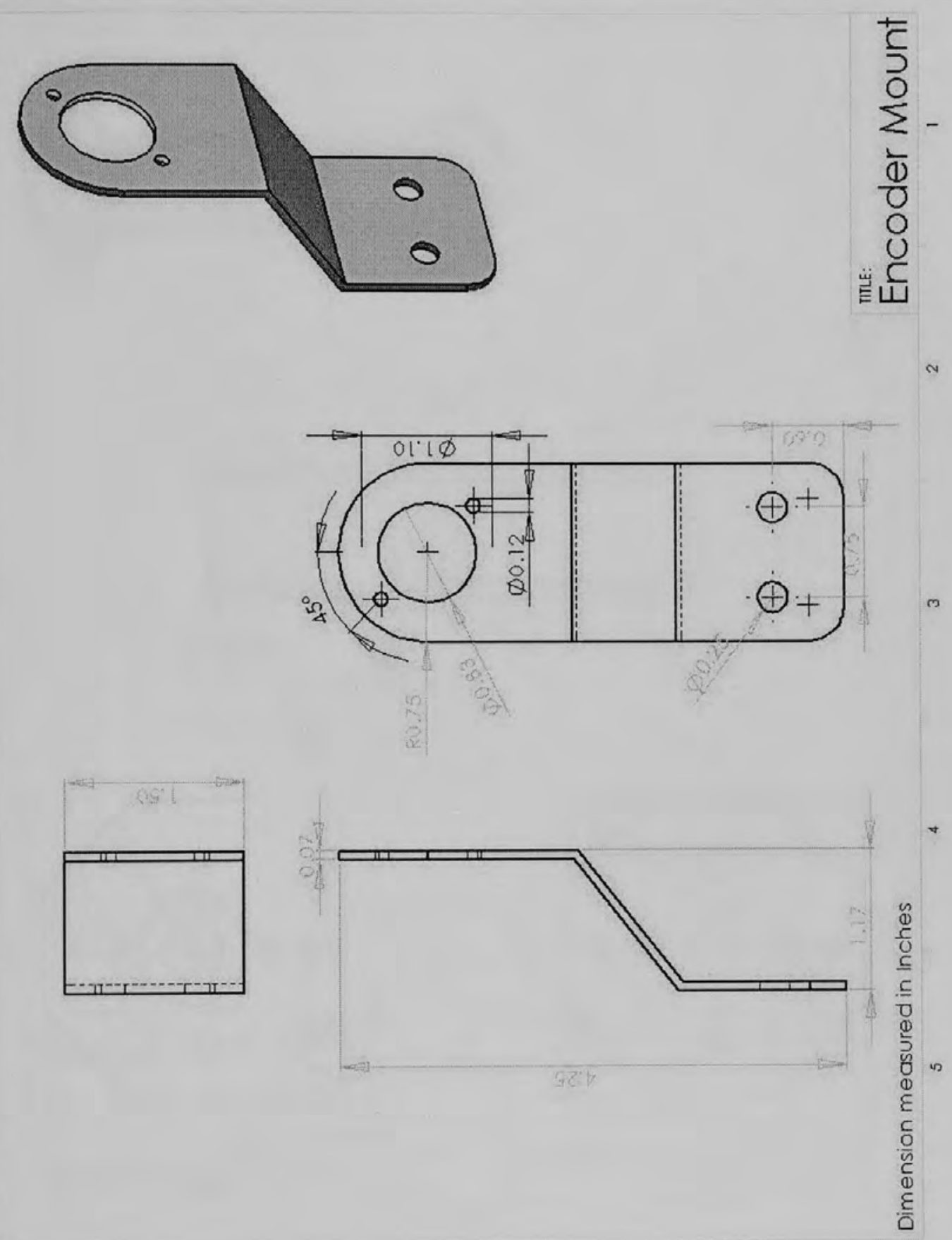

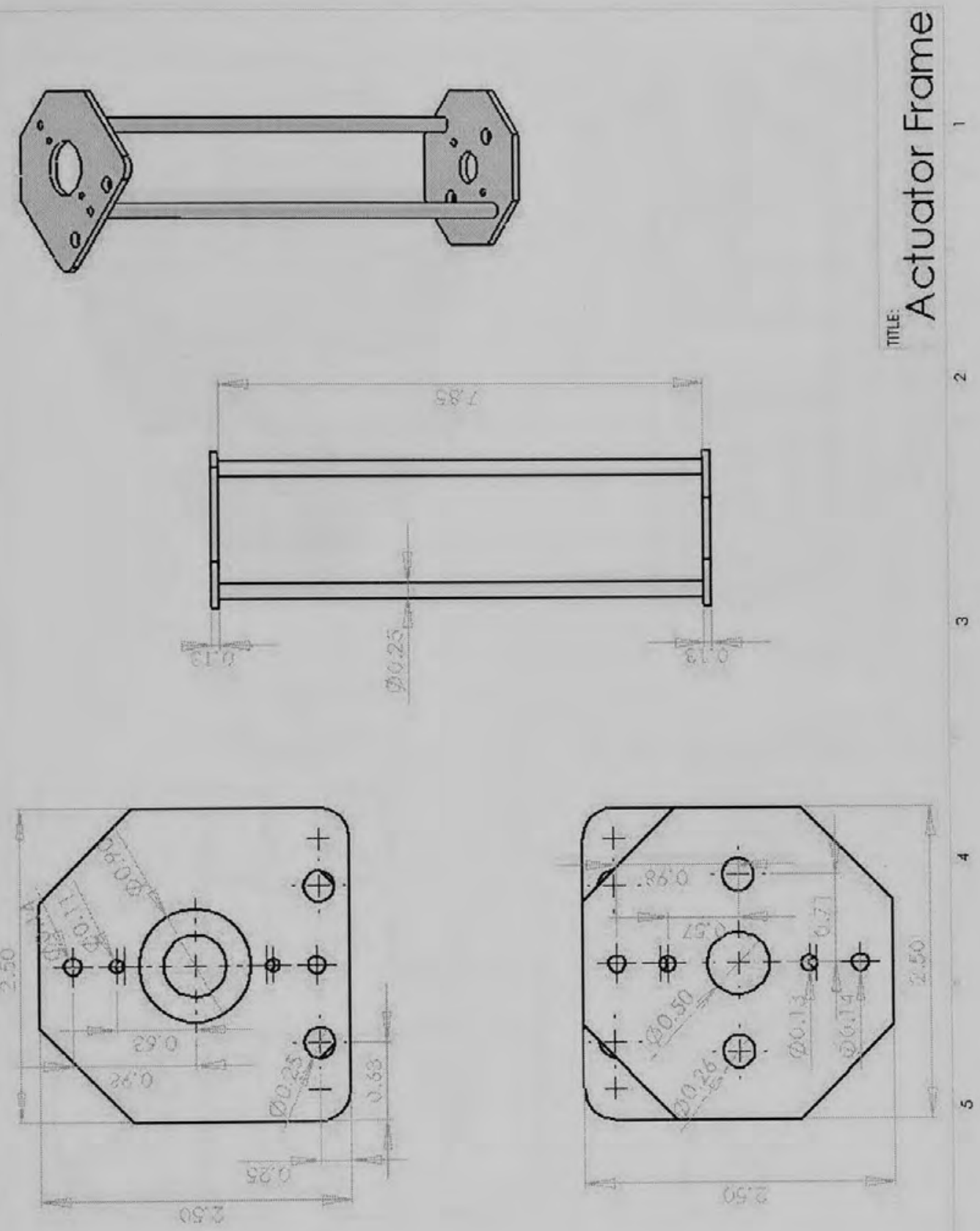

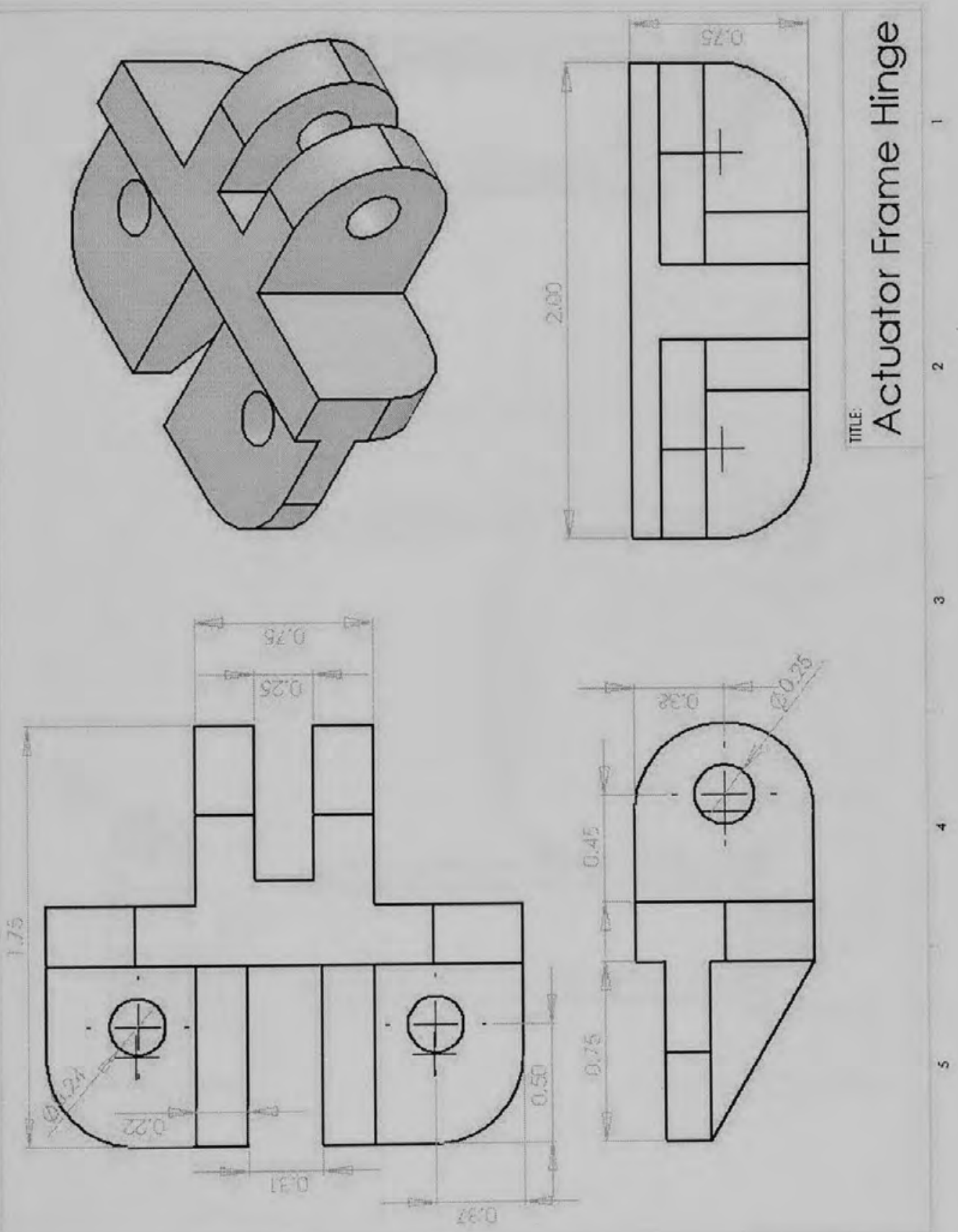

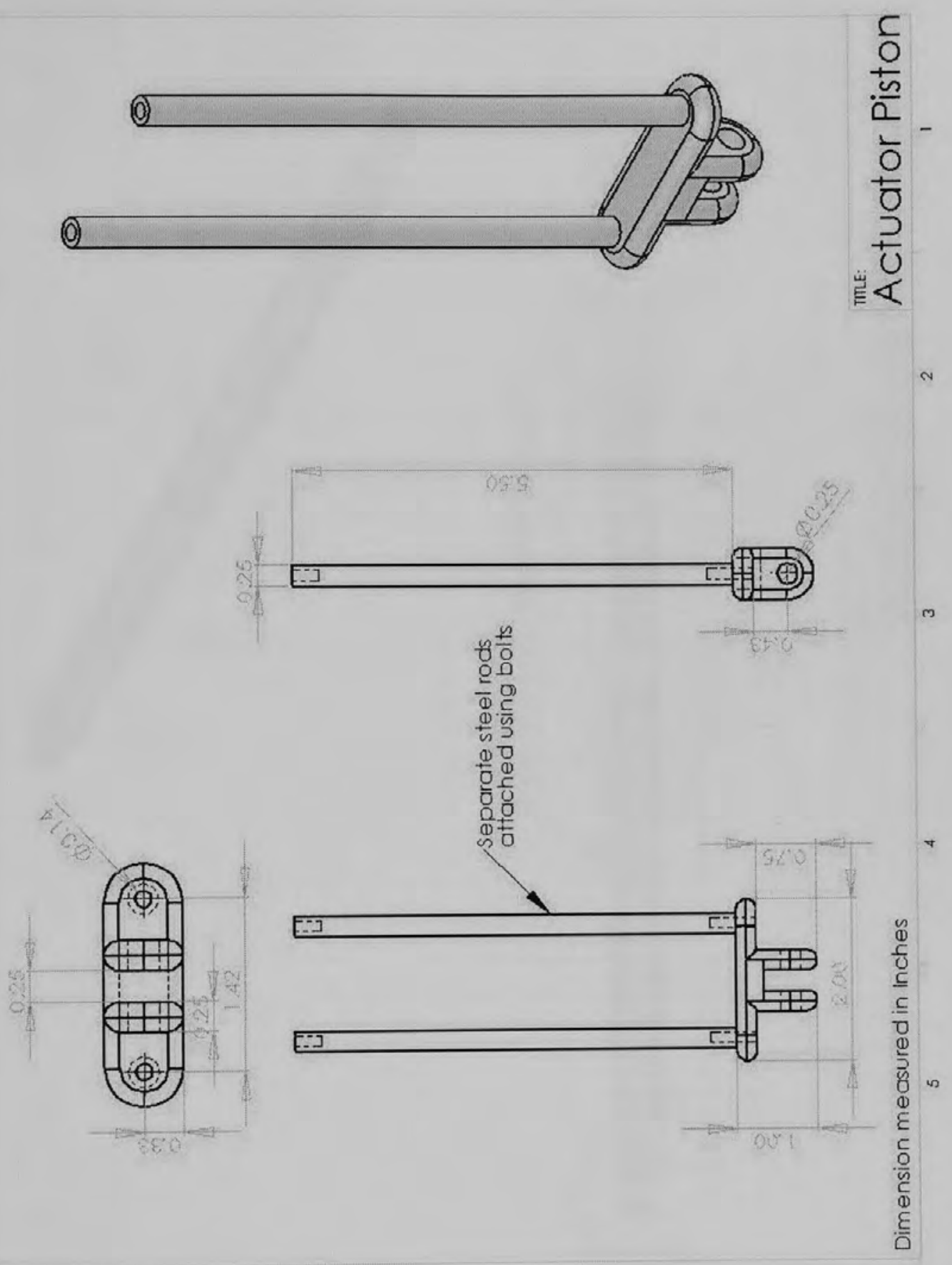


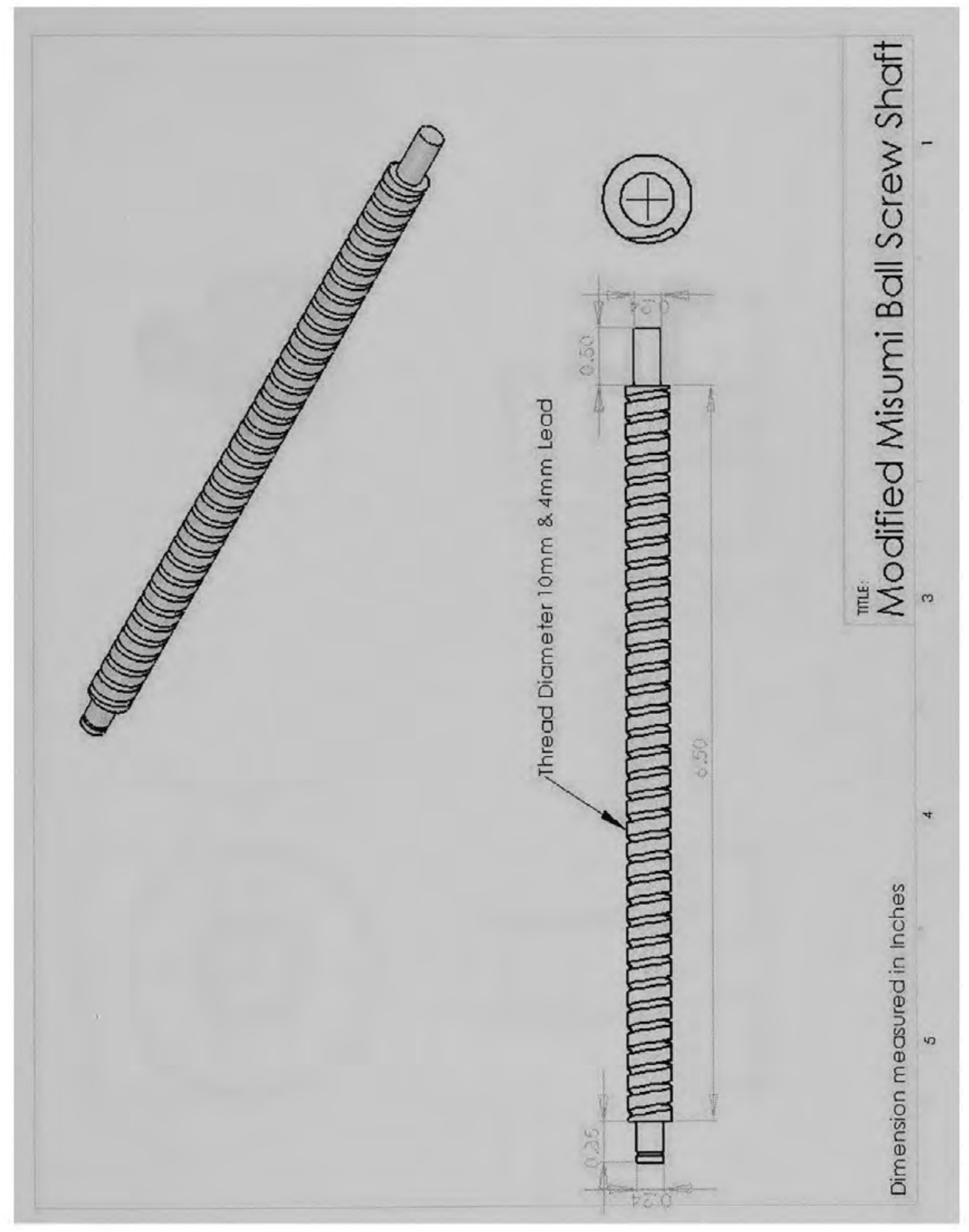




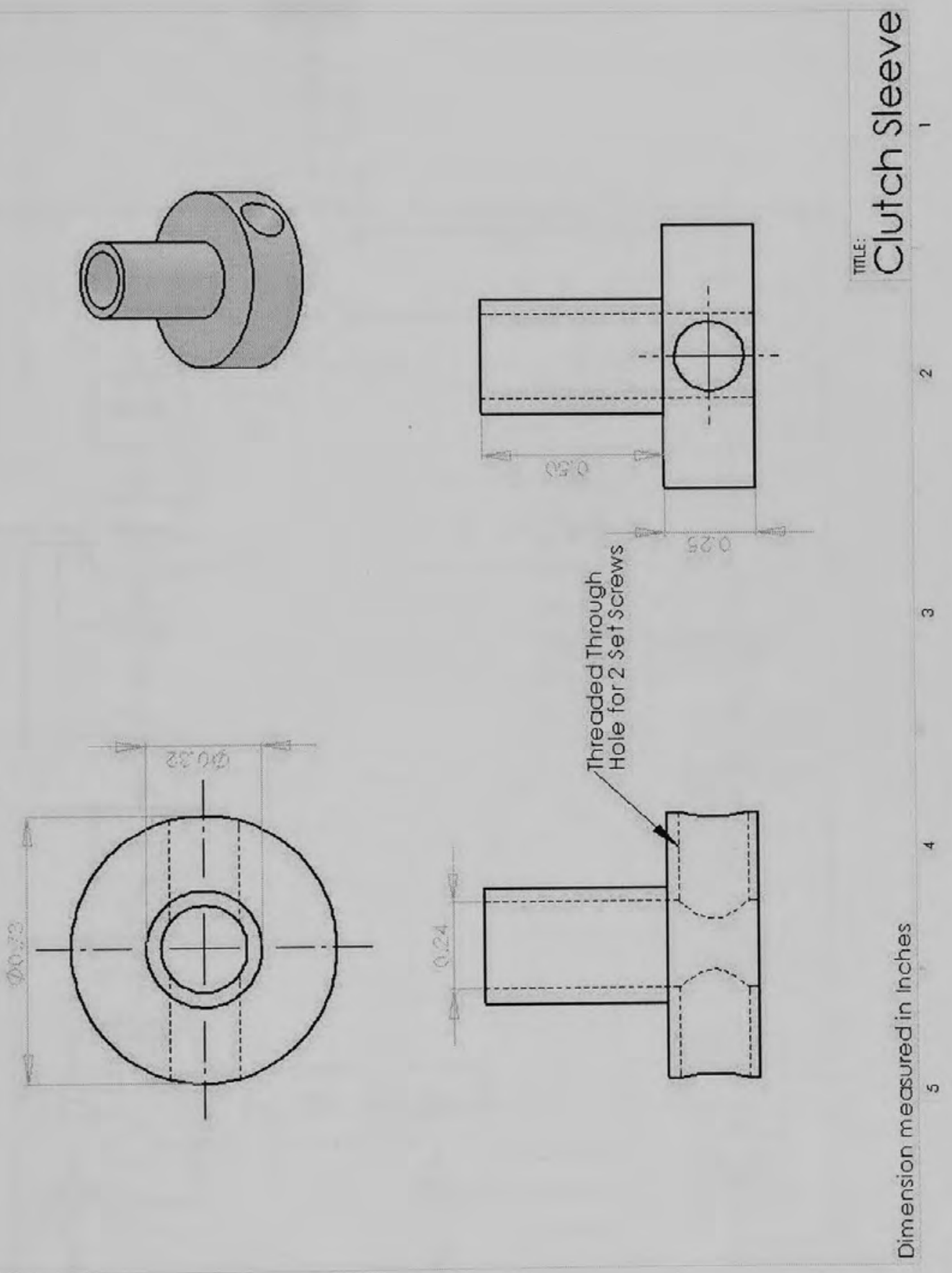


Appendix B: PIC MCU Logic Flowchart

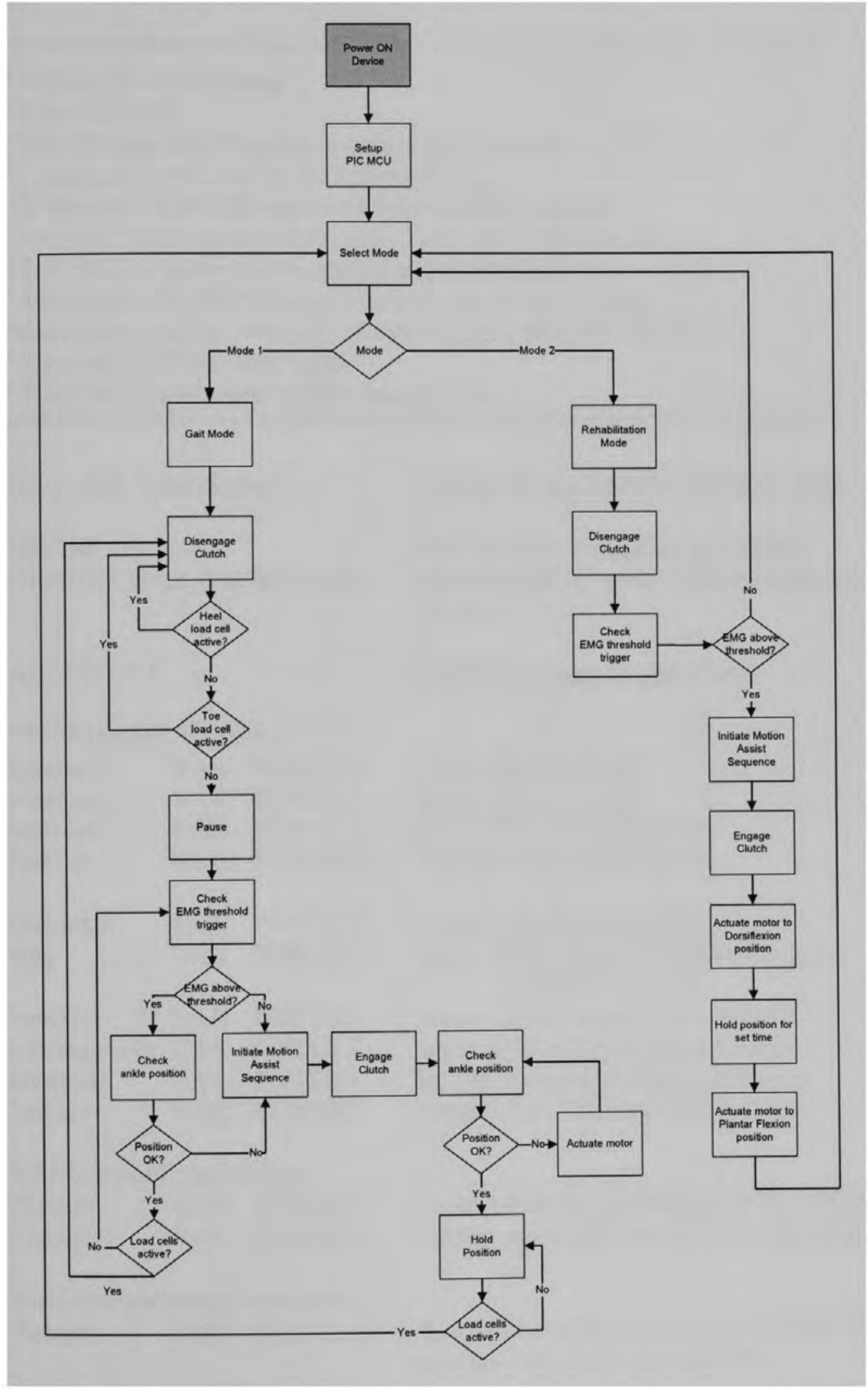


' Author: Szu-Min Chang

' Date: 12/01/06

' BFB Walker Gait Program to output the following:

' 1. status of Load cells to LED (Heel \& Foot)

' 2. turn on clutch LED once foot is lifted off the ground

' 3. check EMG signal to determine if assitmotion is needed

' 4. if EMG is active, check ankle position for clearance

' 5. Includes PIC-SERVO commands to actuate motor/ankle

' 6. Included code to calculate encoder position from PIC-SERVO

' 7. recode LED indicator lights

' 8 . include Dorsiflexion rehabilitation mode

1************************************************************

INCLUDE "bs1defs.bas"

DEFINE OSC 12

DEFINE CHAR_PACING 1000

$\mathrm{ADCON} 1=7$

****** Declare Variables $* * * * *$

loadHeel VAR PORTA.0

ledHeel VAR PORTA.2

ledFoot VAR PORTA.3

ledClutch VAR PORTB.0

emg

VAR PORTB.1

ledEMG VAR PORTB.2

trainingMode VAR PORTB.3

ledRehab VAR PORTB.4

ledGait
loadFoot VAR PORTA.1

'include file for SERIN/SEROUT cmds

'Set Oscillator frequency to $12 \mathrm{MHz}$ 'provides $400 \mathrm{uS}$ space between bytes for serout

'Set PORTA pins to digital use

'input: Heel load cell

'input: Foot load cell

'input: Heel LED indicator

'output: Foot LED indicator

'output: clutch status LED

'input: EMG signal $(0=$ Below Trigger;

$$
1=\text { Above Trigger) }
$$

'output: EMG signal LED indicator

'input: Gait or Rehabilitation mode

'output: Rehab-Dorsi LED indicator

'output: Gait Training LED indicator

'RS232 Input/Output Ports

SerialIn VAR PORTC.7

SerialOut VAR PORTC.6

'Encoder Positioning Variables

encoder VAR byte[4] 'input: serial IN pin (RX) from PIC-SERVO

'output: serial OUT pin (TX) to PIC-SERVO

'four 8-bit storage variable used to hold the position data from PIC-SERVO 
positionEnc VAR word

$\begin{array}{lll}\text { positionErr } & \text { VAR } & \text { word } \\ & & \\ \text { positionRef } & \text { CON } & 380 \\ \text { positionDiff } & \text { CON } & 40 \\ \text { reference position } & \end{array}$

Timeout CON 100

$1 * * * * *$ Declare Inputs $* * * * *$

INPUT loadHeel

INPUT loadFoot

INPUT emg

INPUT trainingMode 'position variable used to store the Least

Significant 16 bits of position data from PIC-SERVO

'difference in position between encoder and check

'acceptable reference position for ankle

'allowable difference in position value compared to

'***** 'Setup' Subroutine to perform initial setup of PIC \& PIC-SERVO at power-up Setup:

PAUSE $500 \quad$ 'Pause to allow proper powerup sequencing

'Setup PIC-SERVO parameters

'PIC-SERVO Module Address: individual=01, group=FF

SEROUT2 SerialOut,32,[\$AA, \$00,\$21,\$01,\$FF,\$21]

PAUSE 1

'Define PIC-SERVO format of return status packet: include position data

SEROUT2 SerialOut,32,[\$AA,\$01,\$12,\$01,\$14]

PAUSE 1

'Reset PIC-SERVO Encoder Start Position to ' 380 '

SEROUT2 SerialOut,32,[\$AA,\$01,\$50,\$02,\$7C,\$01,\$00,\$00,\$D0]

Pause 1

'Set PIC-SERVO PID servo filter operating parameters:

$' \mathrm{Kp}=1400, \mathrm{Kd}=4000, \mathrm{Ki}=70, \mathrm{IL}=1000, \mathrm{OL}=255, \mathrm{CL}=255, \mathrm{EL}=4000, \mathrm{SR}=1, \mathrm{DB}=0, \mathrm{SM}$ $=1$

SEROUT2

SerialOut,32,[\$AA, $\$ 01, \$ F 6, \$ 78, \$ 05, \$ A 0, \$ 0 F, \$ 46, \$ 00, \$ E 8, \$ 03, \$ F F, \$ F F, \$ A 0, \$ 0 F, \$ 01, \$$ $00, \$ 01, \$ 03]$

\section{PAUSE 1}

'Enable PIC-SERVO Amplifier: Enable Amp only SEROUT2 SerialOut,32,[\$AA,\$01,\$17,\$01,\$19] 
PAUSE 1

'Reset encoder position variables on PIC at powerup

positionEnc $=0$

positionErr $=0$

GOTO Mode

* 'Mode' Subroutine to check which mode system will be used for

Mode 1: Gait training to correct foot drop

Mode 2: Rehabilitation to train Dorsiflexion

Mode:

IF trainingMode $=1$ THEN

HIGH ledGait

LOW ledRehab

GOTO GaitMain 'Goto Gait rehabilitation mode

ENDIF

LOW ledGait

HIGH ledRehab

GOTO DorsiMain 'Goto Dorsiflexion rehabiliation mode

GOTO Mode

'***** 'GaitMain' Subroutine to check when the foot makes contact to the floor during $1 * * * * *$ gait. At which time, system disengages clutch and motor to allow user to freely 1***** move ankle with minimum resistance

GaitMain:

IF loadHeel $=1$ THEN 'Keep clutch/motor disengaged if Heel load cell is active

'DEBUG

SEROUT2 SerialOut,32,["Heel on ground",13]

LOW ledClutch

HIGH ledHeel

'Show Heel on floor

LOW ledEMG

GOSUB StopMotor 
PAUSE 400

\section{ENDIF}

GOTO Mode
IF loadFoot $=1$ THEN $\quad .$. 'Keep clutch/motor disengaged if Foot load cell is
'DEBUG active
SEROUT2 SerialOut,32,["Foot on ground",13]

LOW ledClutch

HIGH ledFoot

'Show Foot on floor

LOW ledEMG

GOSUB StopMotor

PAUSE 200
GOTO Mode
ENDIF

LOW ledHeel

LOW ledFoot

GOTO Checkemg

GOTO Mode

'***** 'Checkemg' Subroutine to check the input from the EMG device to determine if $1 * * * * *$ user is initiating muscle movement and if proper ankle position is $1 * * * * *$ achieved for foot clearance

Checkemg:

\section{'DEBUG:}

1 $\quad$ SEROUT2 SerialOut,32,["Checking EMG Reading",13]

IF emg $=1$ THEN

'if EMG is above threshold, proceed to check ankle position

'DEBUG:

SEROUT2 SerialOut,32,["EMG reading is = 1",13,"Retreive Position from PIC-SERVO",13]

HIGH ledEMG 
'Send command to PIC-SERVO to return position data

SEROUT2 SerialOut,32,[\$AA,\$01,\$13,\$01,\$15]

'Waits for Status return byte $79 \mathrm{~h}$ and stores the next four position data into 4 separate variables

SERIN2

SerialIn,32,Timeout,Mode,[WAIT(\$79),encoder(0),encoder(1),encoder(2),encoder(3)]

'SERIN2 SerialIn,32,[WAIT("79"),HEX2 encoder(0),HEX2

encoder(1),HEX2 encoder(2),HEX2 encoder(3)]

'DEBUG:

SEROUT2 SerialOut,32,["Position from PIC-SERVO Received",13]

'Calculate ankle position from encoder

'Store first byte of position data into overall position variable (i.e. 00XX)

positionEnc $=$ positionEnc + encoder $(1)$

'Shift position variable 8 bits to the left (i.e. XX00)

positionEnc $=$ positionEnc $<<8$

'Append LSB 8-bit value to position variable (i.e. XXYY)

positionEnc $=$ positionEnc + encoder $(0)$

'DEBUG:

SEROUT2 SerialOut,32,["Encoder Position = ",DEC positionEnc,13]

'Calculate the absolute value of position difference between reference and current position

positionErr $=\mathrm{ABS}($ positionRef - positionEnc)

'DEBUG:

SEROUT2 SerialOut,32,["Position Error = ",DEC positionErr,13]

'Initiate movement if error value is above acceptable difference

IF positionErr $>$ positionDiff THEN

'DEBUG:

SEROUT2 SerialOut,32,["Error Greater than allowable",13]

GOSUB ResetPos

GOTO GaitAssistmotion 'if ankle position if inadequate,

proceed to help assist sequence

ENDIF 
IF loadHeel = 1 THEN GOTO GaitMain 'check to see if foot makes contact to ground

IF loadFoot $=1$ THEN GOTO GaitMain

'DEBUG:

OK!",13]

SEROUT2 SerialOut,32,["Error SMALLER than limit. Position is

GOSUB ResetPos

PAUSE 10

GOTO Checkemg

ENDIF

'DEBUG:

- $\quad$ SEROUT2 SerialOut,32,["EMG reading is $=0 ", 13]$

GOSUB ResetPos

LOW ledEMG

'If no EMG is detected, no movement will result thus proceed directly to assisted motion

GOTO GaitAssistmotion

GOTO GaitMain

'***** 'GaitAssist' Subroutine to initiate automated assist sequence to reposition the $1 * * * * *$ user foot into the proper position to achieve foot clearance

GaitAssistmotion:

'DEBUG:

SEROUT2 SerialOut,32,["Initiate Assit-Motion",13]

HIGH ledClutch 'Engage clutch to initate motion between motor and actuator

'Load PIC-SERVO trajectory information and start motion:

'Position=380,Velocity=15,000,000,Acceleration=500,Trap Profile,Start motion SEROUT2

SerialOut,32,[\$AA, \$01,\$D4,\$97,\$7C,\$01, \$00,\$00,\$C0,\$E1,\$E4,\$00,\$F4,\$01, \$00,\$00,\$6 3] 
PAUSE 10

IF loadHeel = 1 THEN GOTO GaitMain 'check to see if foot makes

contact to ground

IF loadFoot $=1$ THEN GOTO GaitMain

PAUSE 10

GOTO GaitAssistmotion

'***** 'StopMotor' Subroutine send command to PIC-SERVO to

$1 * * * * *$ disengage motor from attempting to keep PID position

StopMotor:

'DEBUG:

SEROUT2 SerialOut,32,["Stop Motor",13]

'Disengage Motor, keep amplifier enabled

SEROUT2 SerialOut,32,[\$AA,\$01,\$17,\$03,\$1B]

RETURN

'***** 'ResetPos' Subroutine to zero out variables

ResetPos:

positionEnc $=0$

positionErr $=0$

'DEBUG:

' SEROUT2 SerialOut,32,["Reset Encoder Pos = ",DEC positionEnc," and Encoder Err $=$ ",DEC positionErr,13]

\section{RETURN}

'***** 'DorsiMain' Subroutine for Dorsiflexion Rehabilitation Mode

$1 * * * * *$ Reads EMG signal strength for trigger threshold

$1 * * * * *$ When triggered, system automatically moves foot into Dorsiflexion position

$1 * * * * *$ and holds position for specified duration.

****** System automatically moves foot to plantar flexion position and release clutch

$1 * * * * *$ awaits next trigger input

DorsiMain: 
'DEBUG:

' SEROUT2 SerialOut,32,["Rehab Mode",13]

LOW ledClutch

LOW ledHeel

LOW ledFoot

LOW ledEMG

IF emg $=1$ THEN

motor and actuator

HIGH ledClutch 'Engage clutch to initate motion between

HIGH ledEMG

'Load PIC-SERVO trajectory information and start motion:

'Position=500,Velocity $=15,000,000$,Acceleration=500, Trap Profile,Start

motion

SEROUT2

SerialOut,32,[\$AA, $\$ 01, \$ D 4, \$ 97, \$ F 4, \$ 01, \$ 00, \$ 00, \$ C 0, \$ E 1, \$ E 4, \$ 00, \$ F 4, \$ 01, \$ 00, \$ 00, \$$

$\mathrm{DB}]$

PAUSE 3000

'Load PIC-SERVO trajectory information and start motion:

'Position=100,Velocity=15,000,000,Acceleration=500,Trap Profile,Start

motion

SEROUT2

SerialOut,32,[\$AA, $\$ 01, \$ D 4, \$ 97, \$ 64, \$ 00, \$ 00, \$ 00, \$ C 0, \$ E 1, \$ E 4, \$ 00, \$ F 4, \$ 01, \$ 00, \$ 00, \$ 4$

A]

PAUSE 1500

GOSUB StopMotor

GOTO Mode

ENDIF

GOTO Mode 
Appendix D: BARA Circuit Diagram

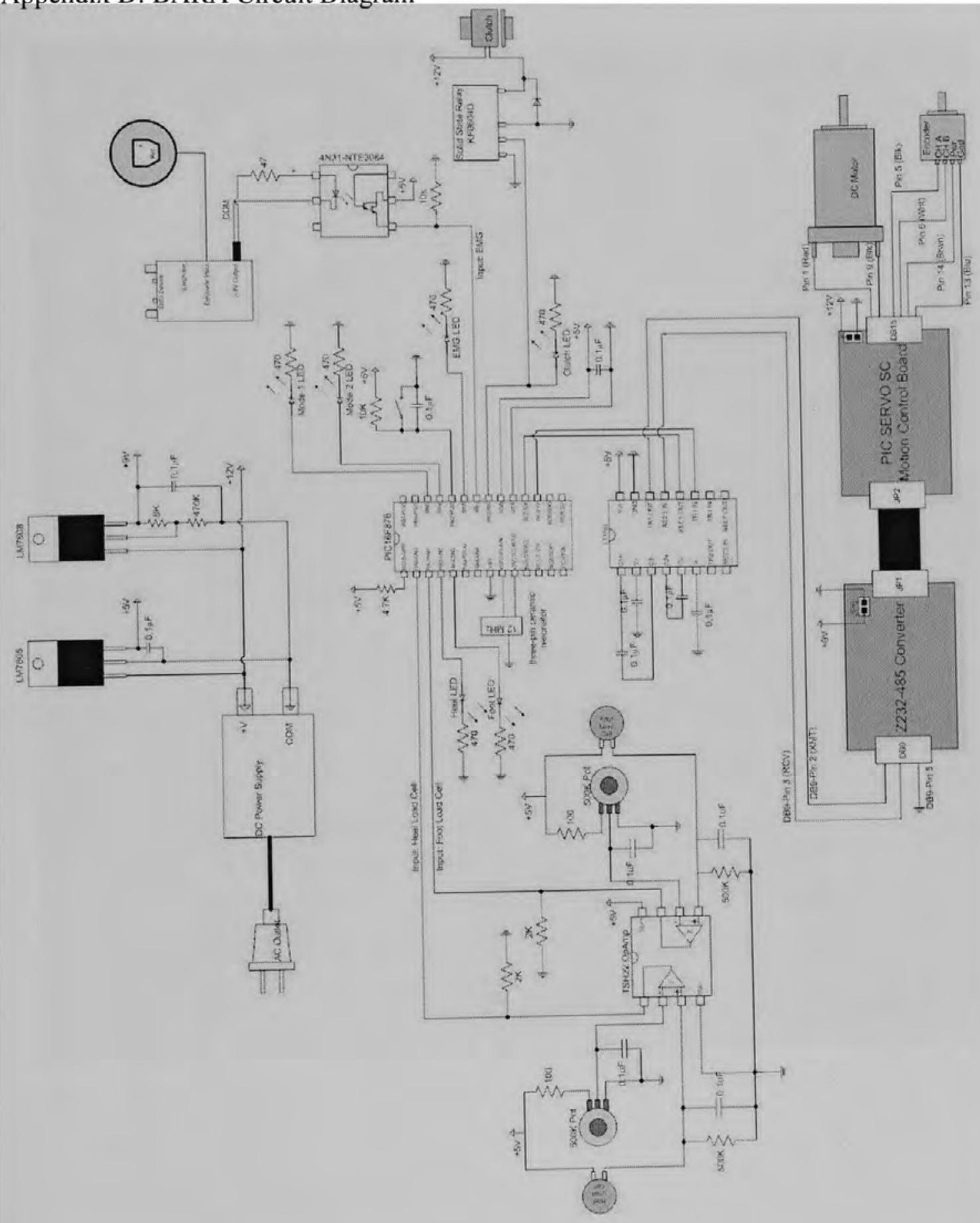


Appendix E: Exploded View Rendering of Actuator and BARA
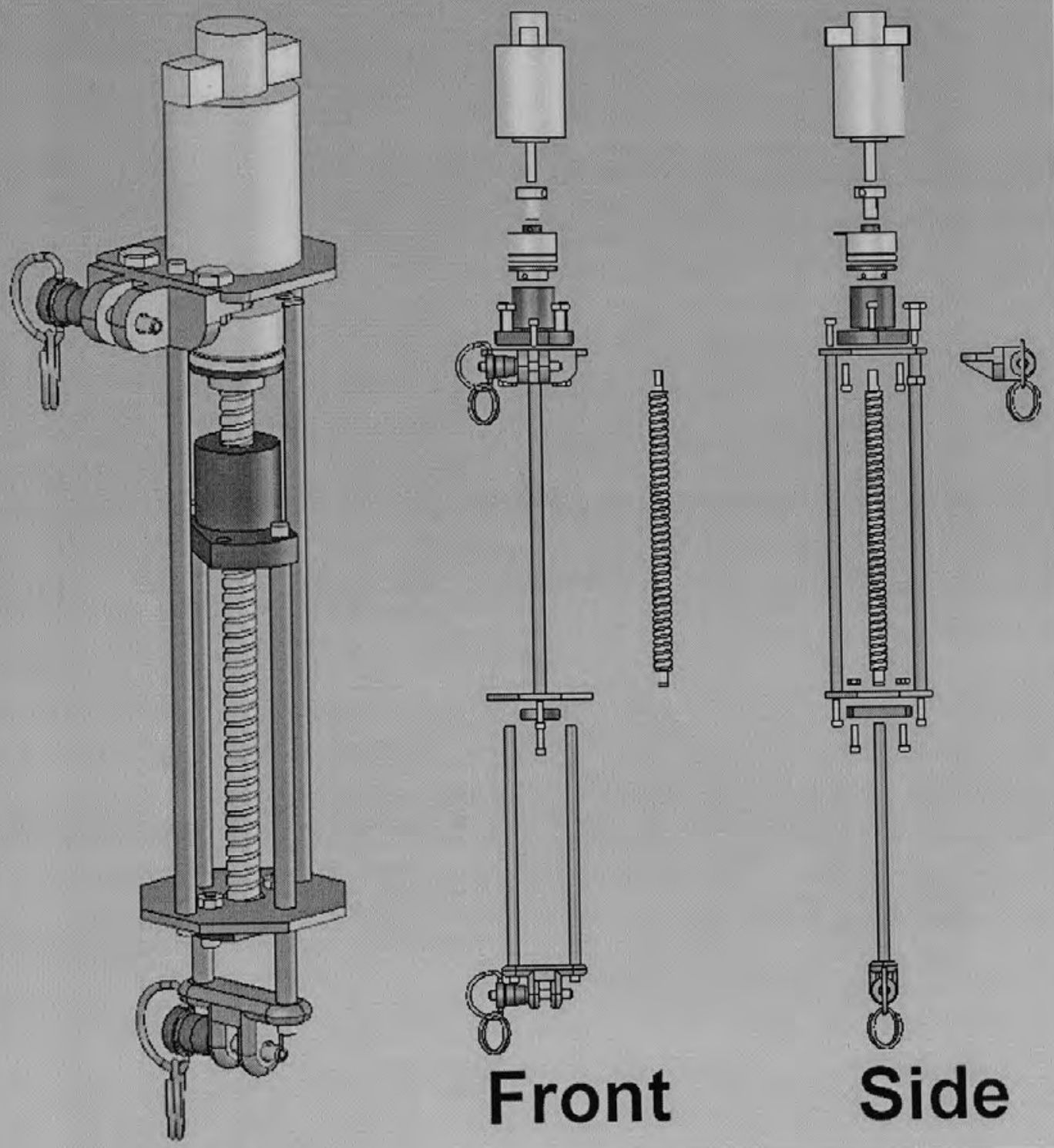


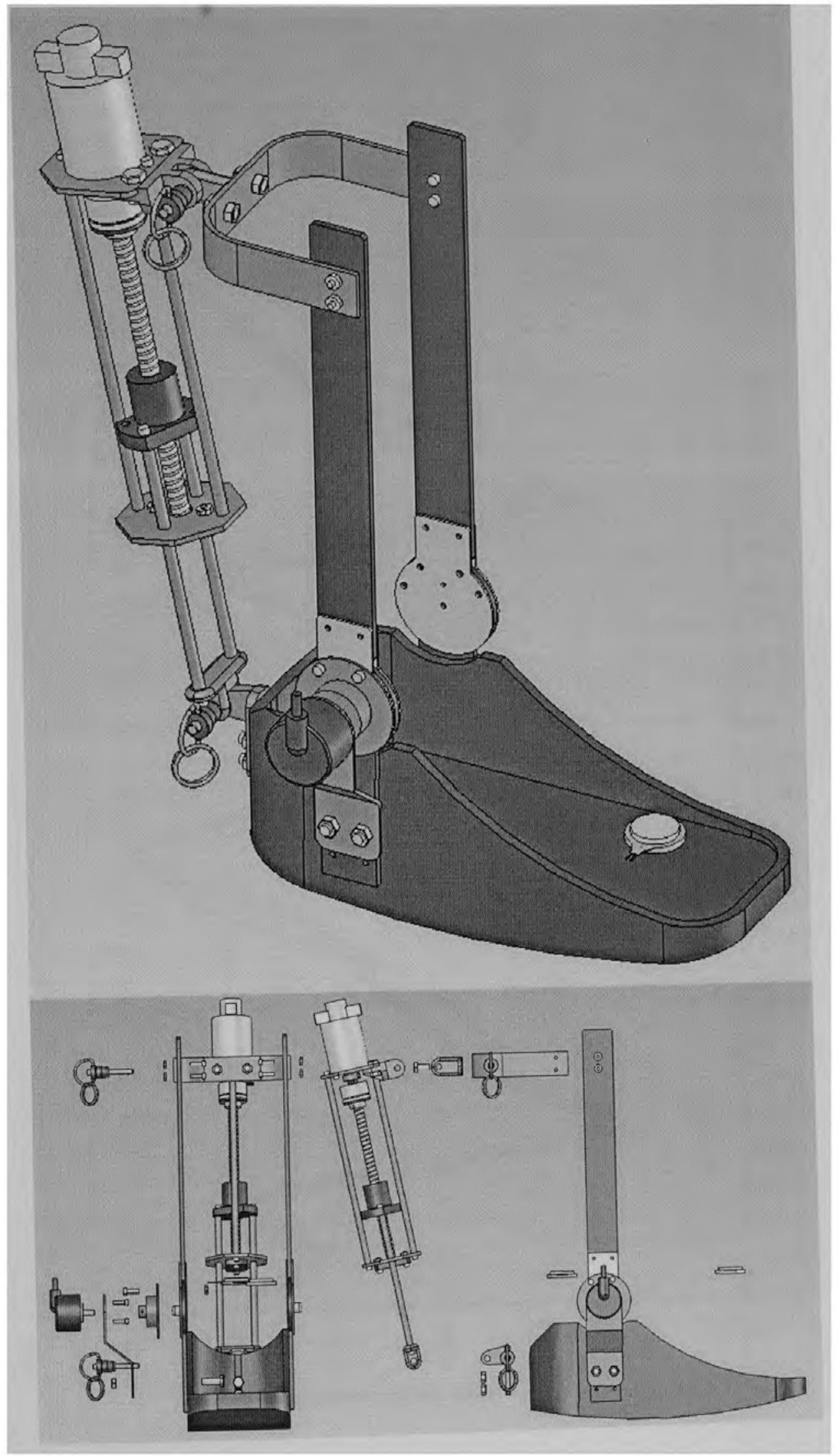


Appendix F: Joint Angle Analysis Graphs

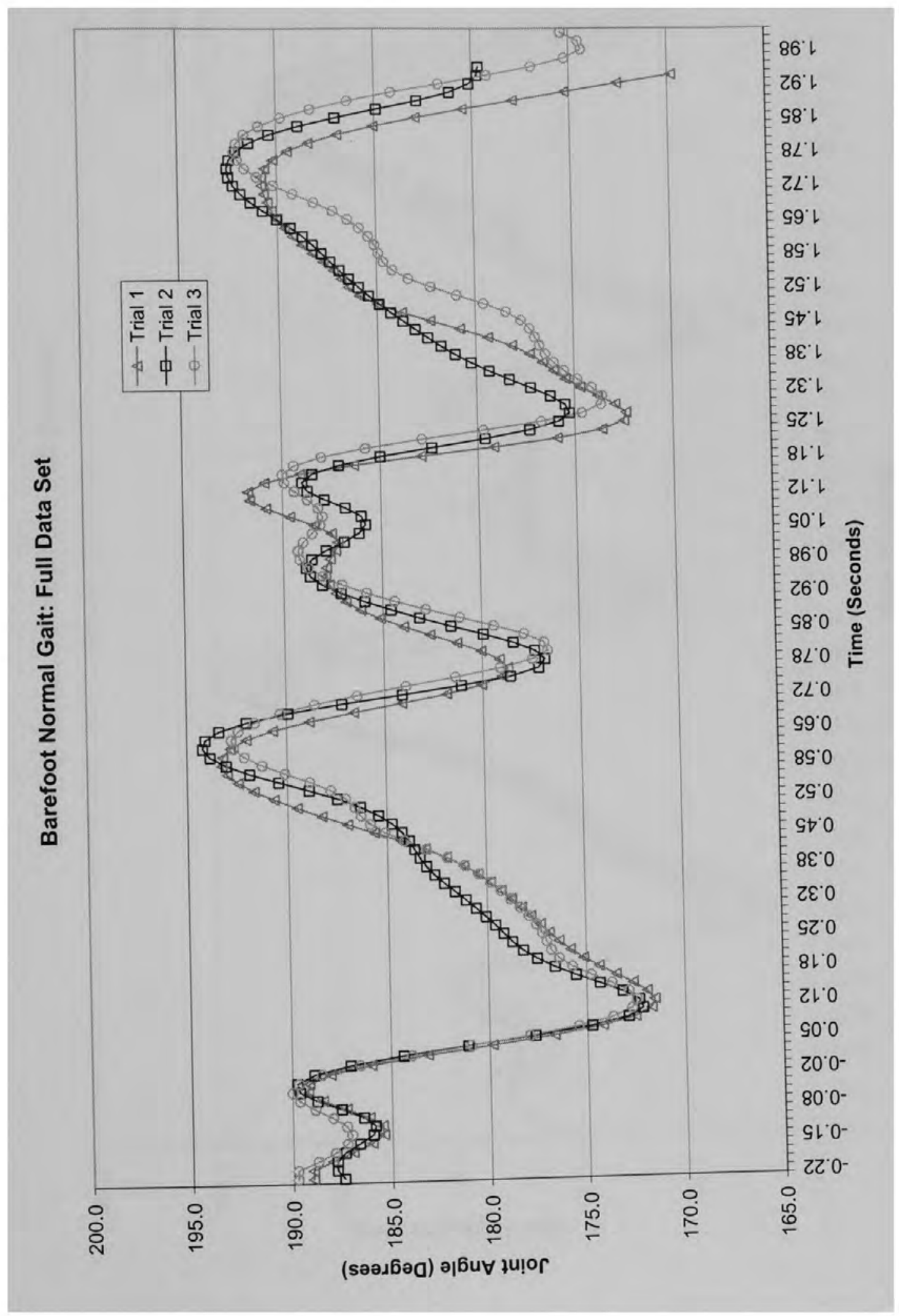




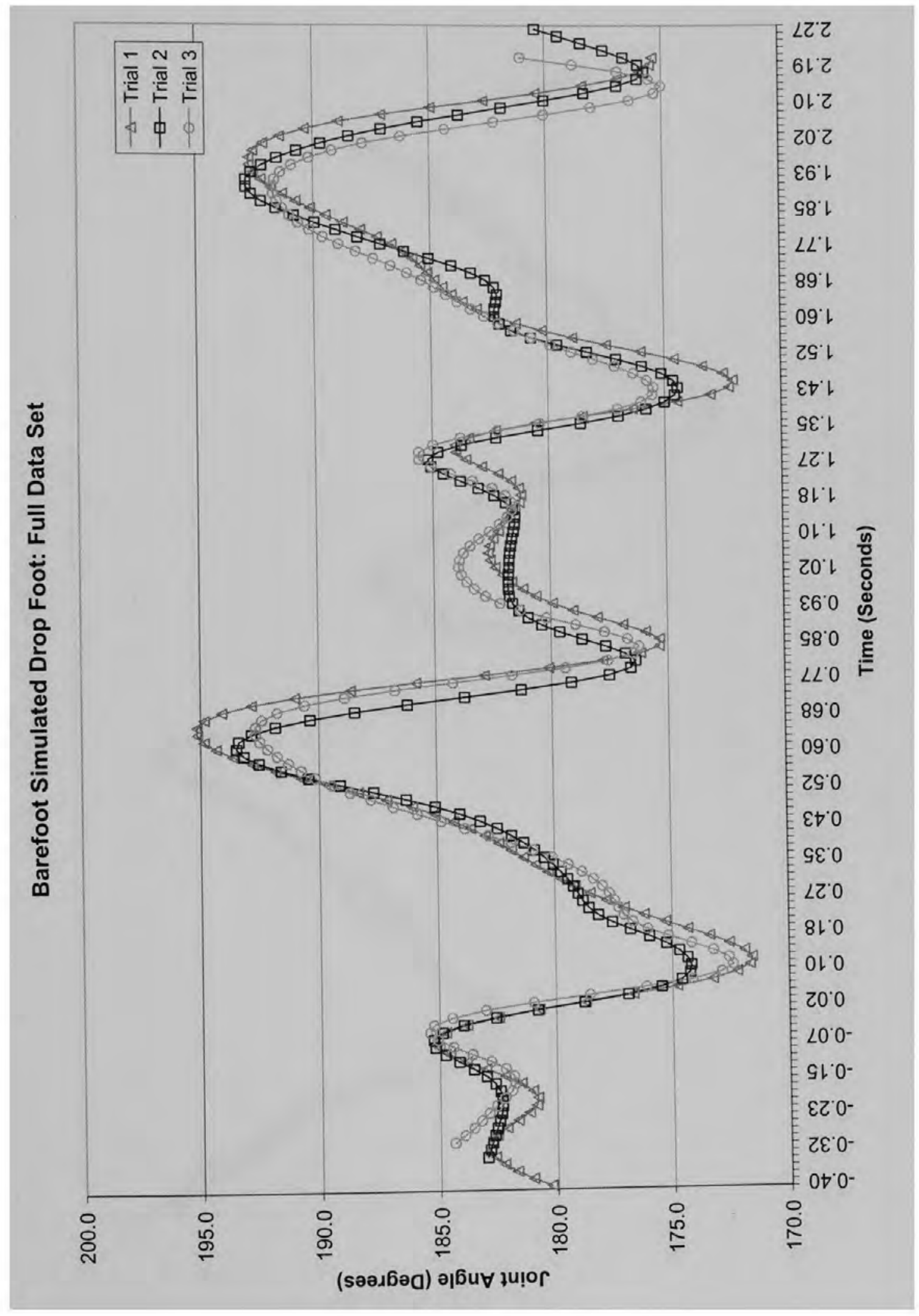




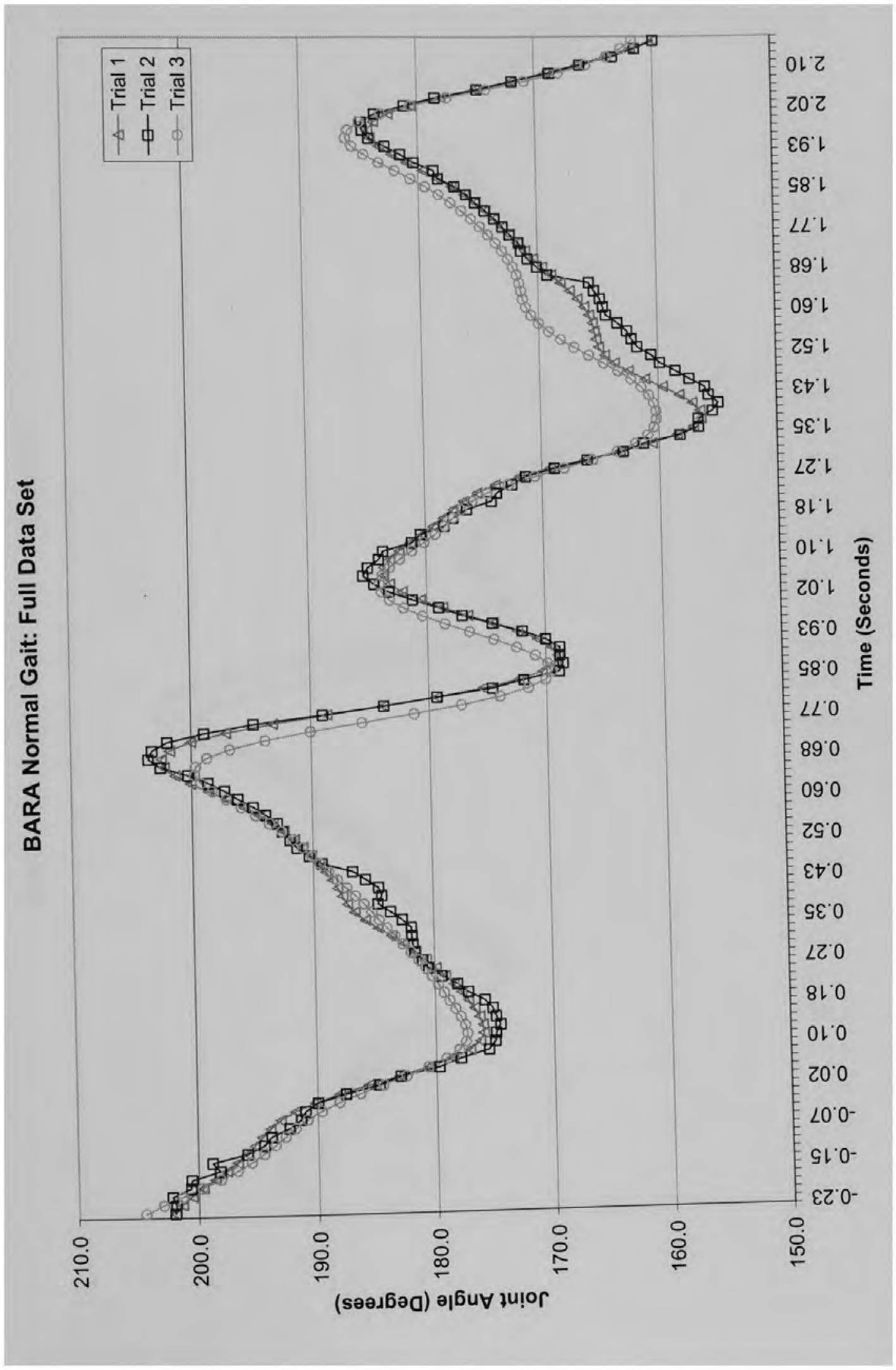




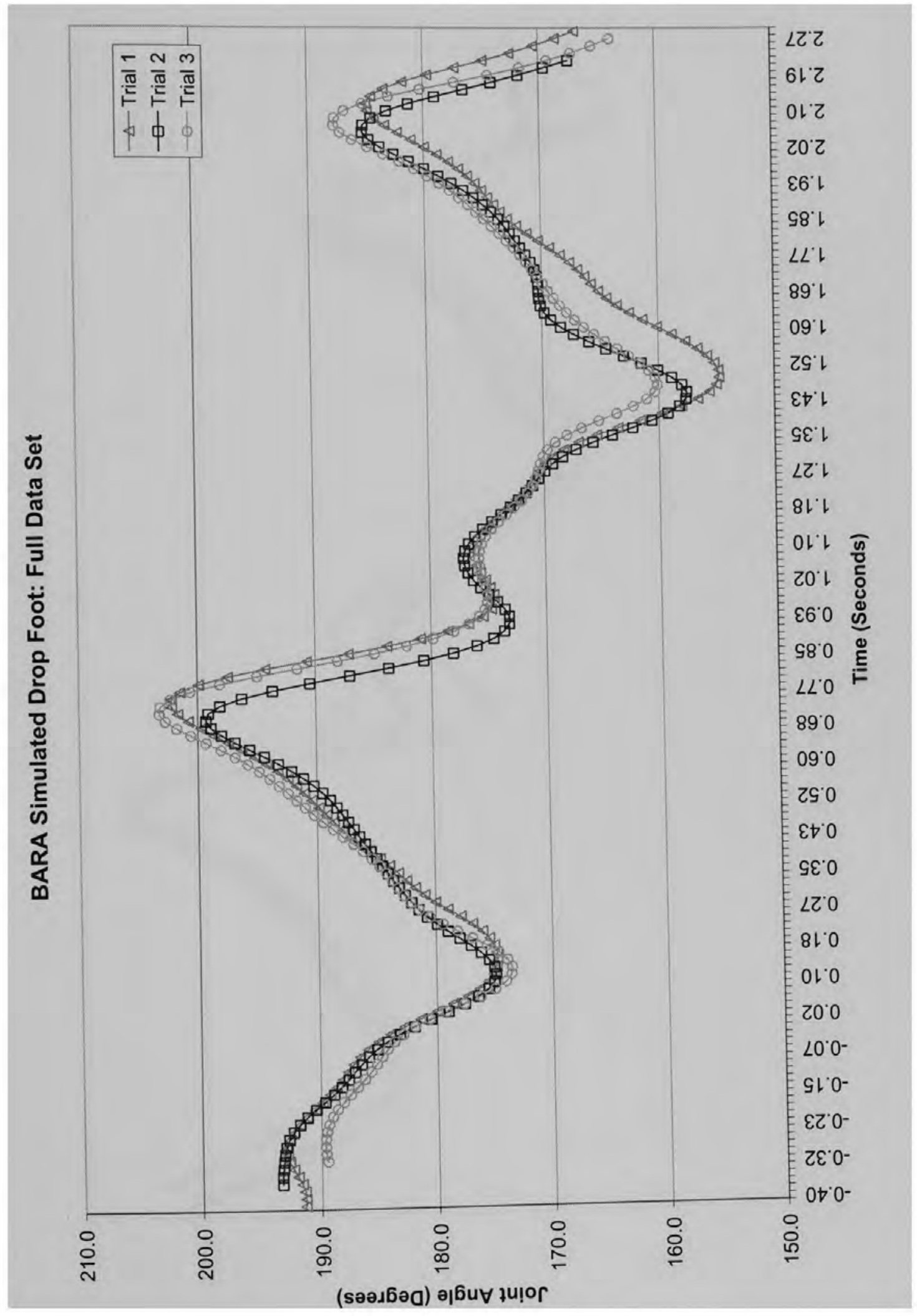




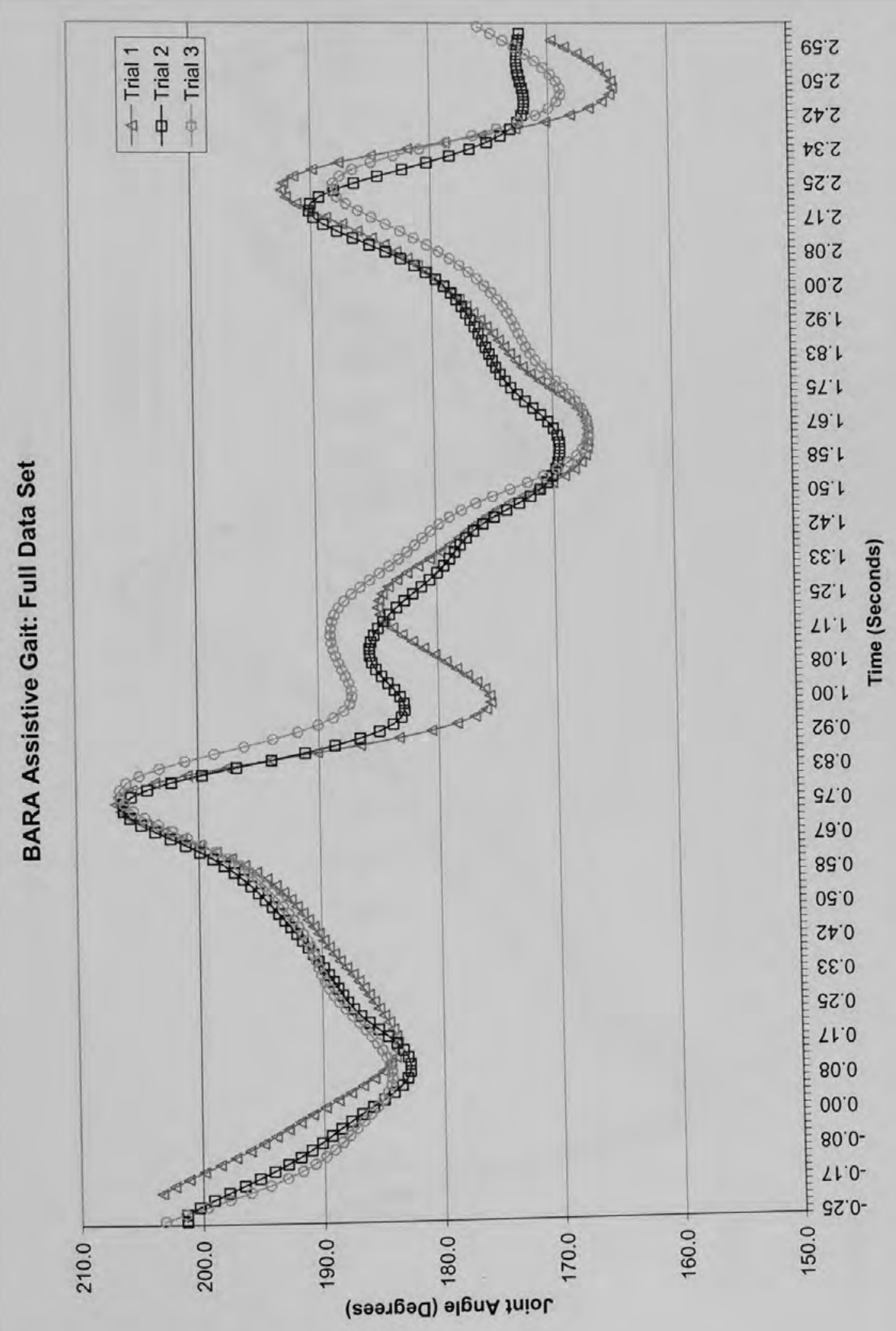




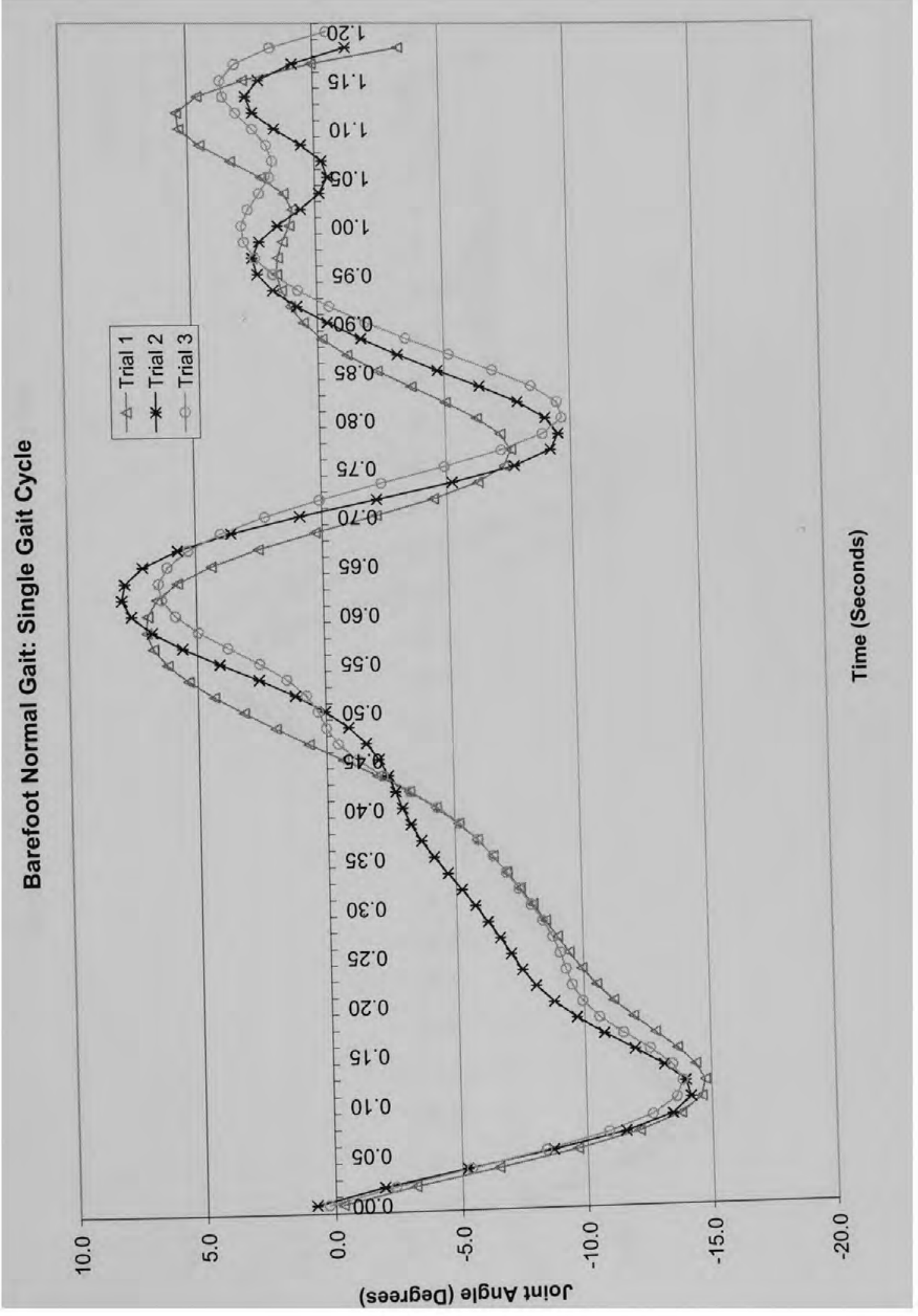




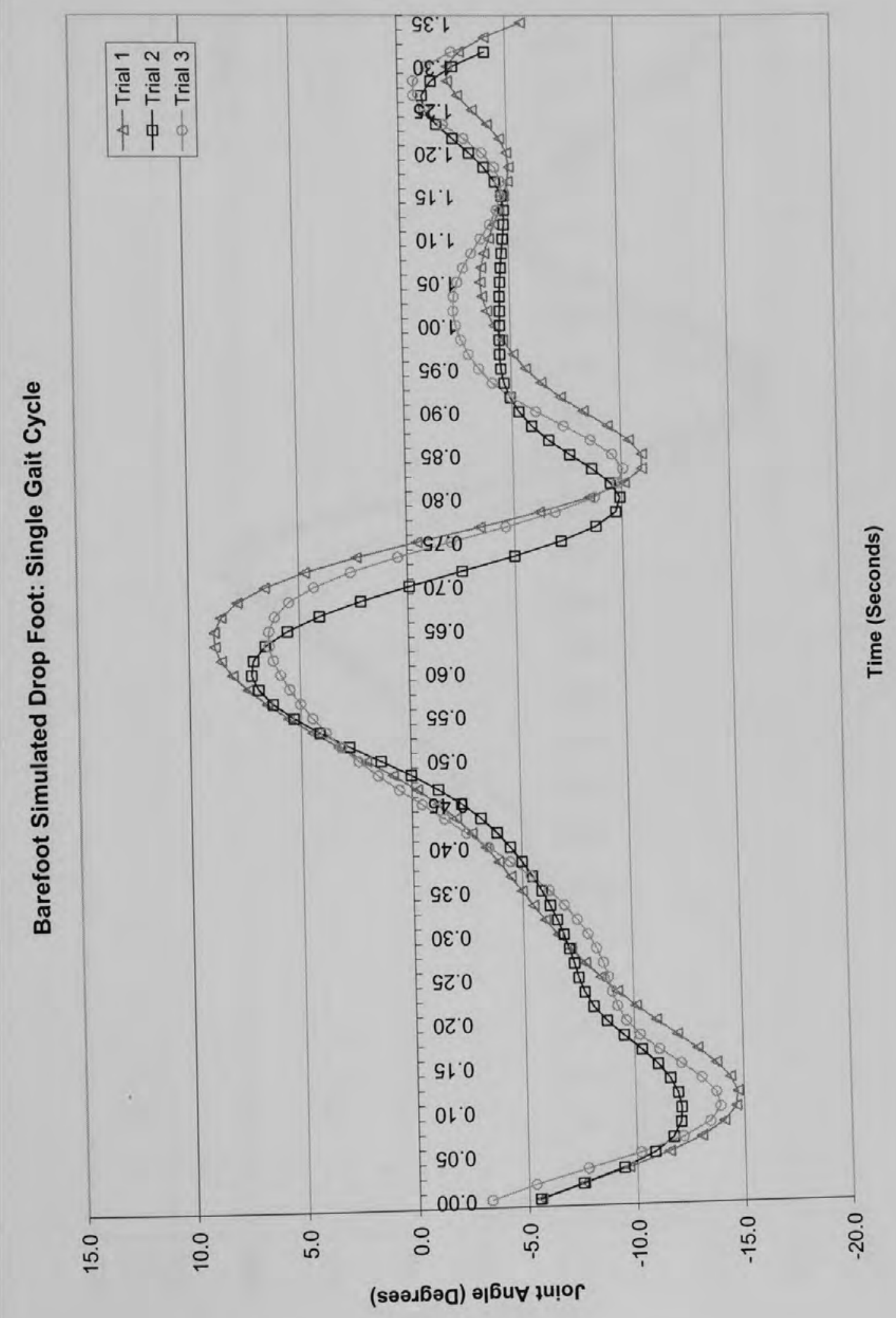




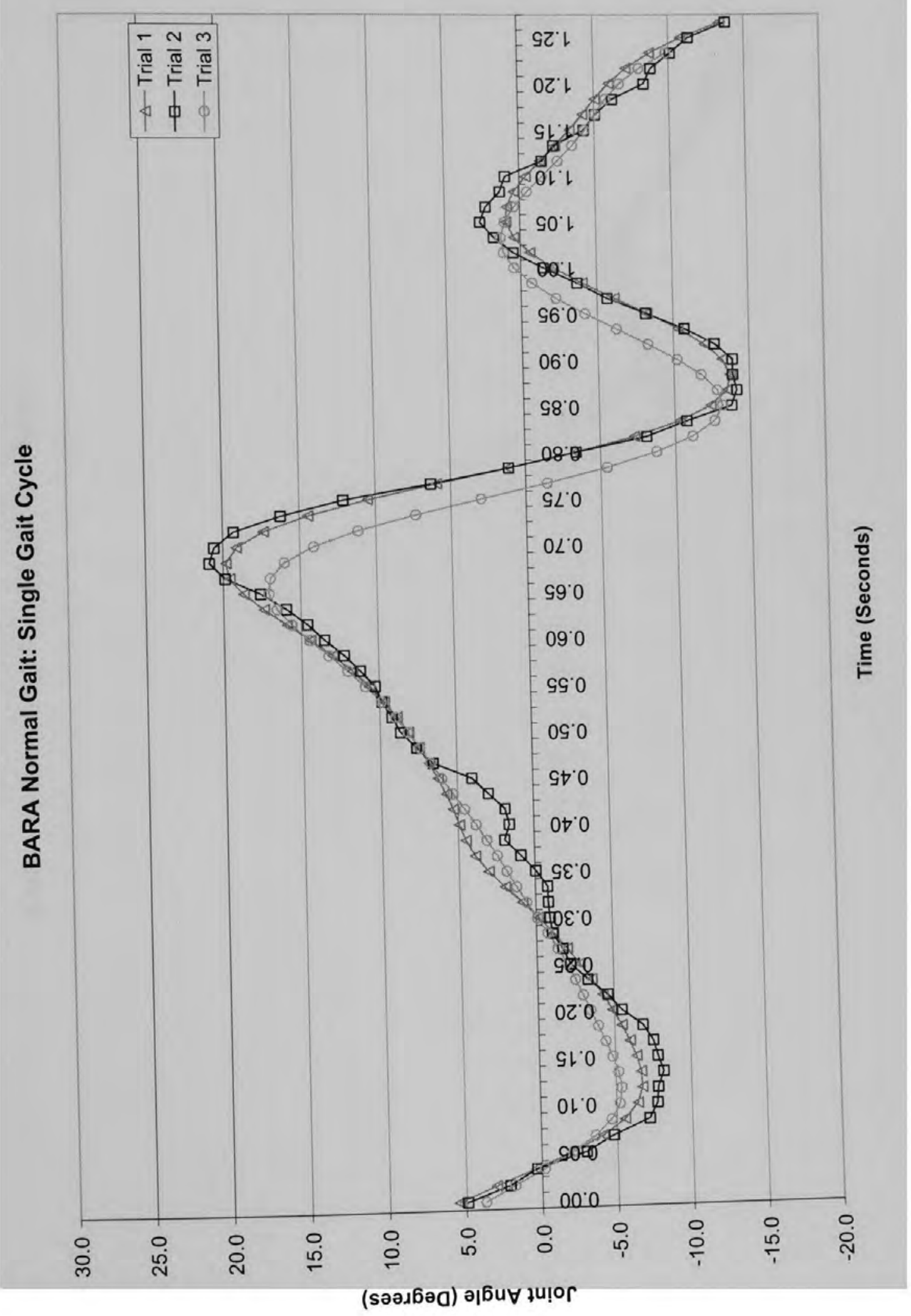




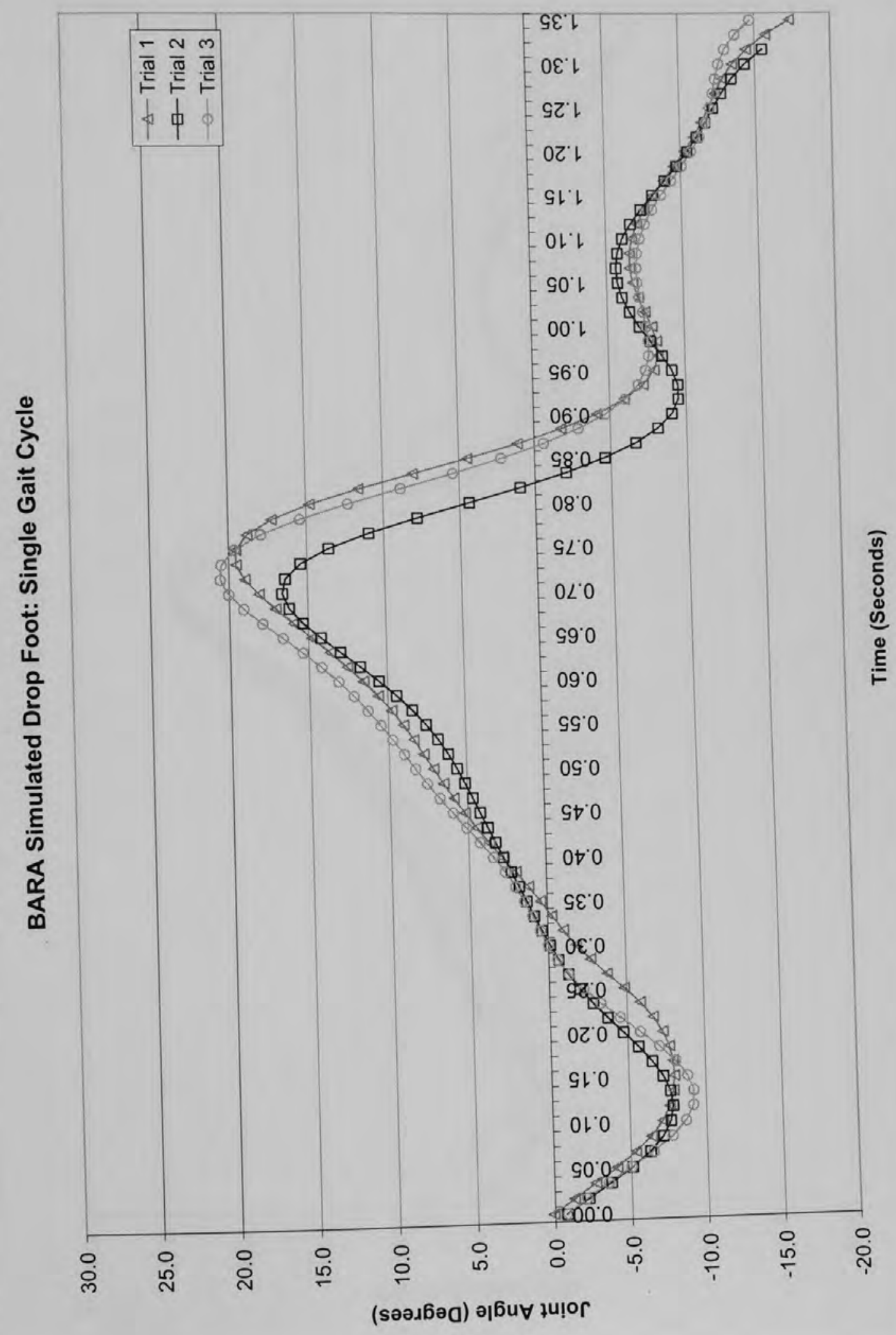




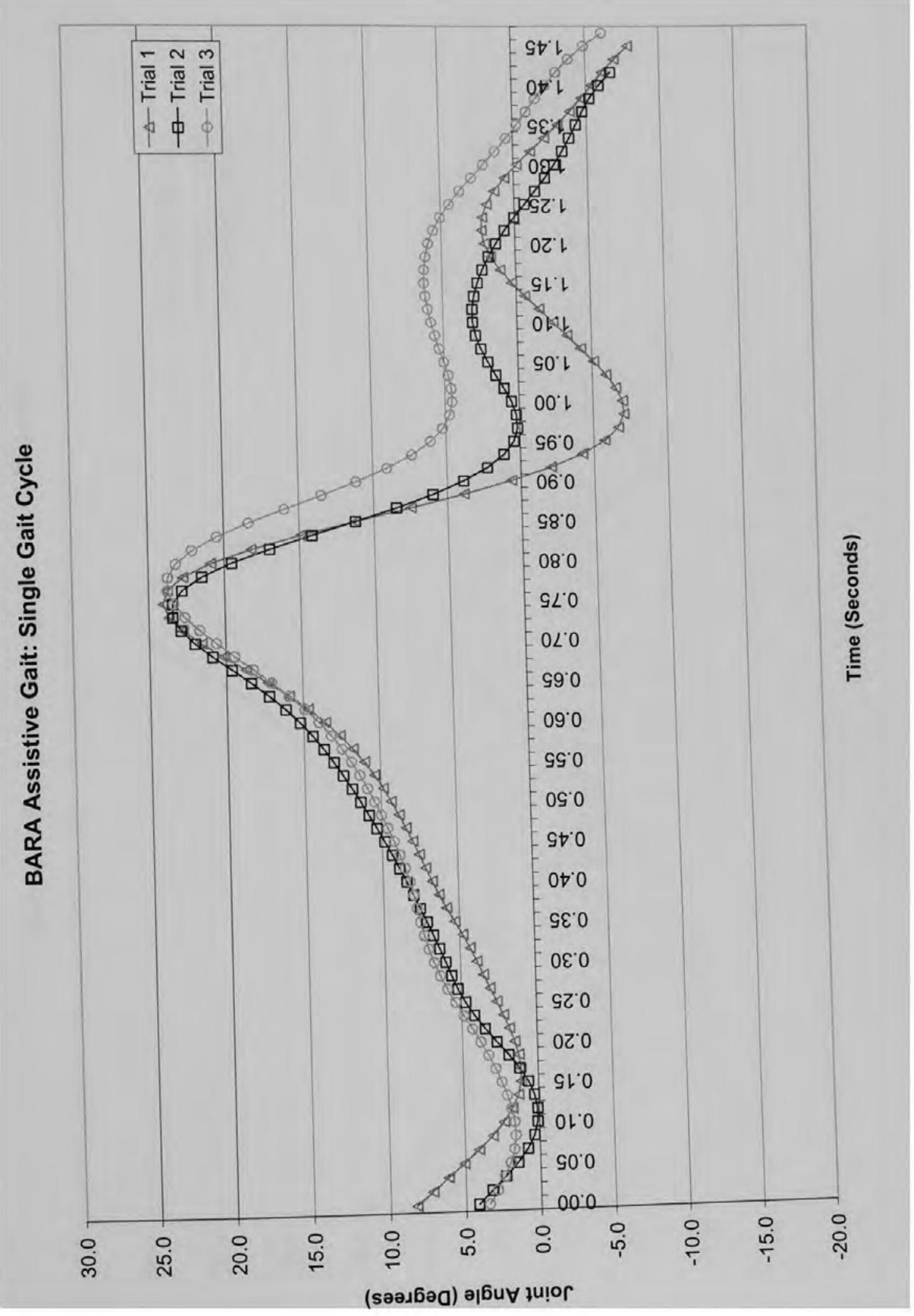




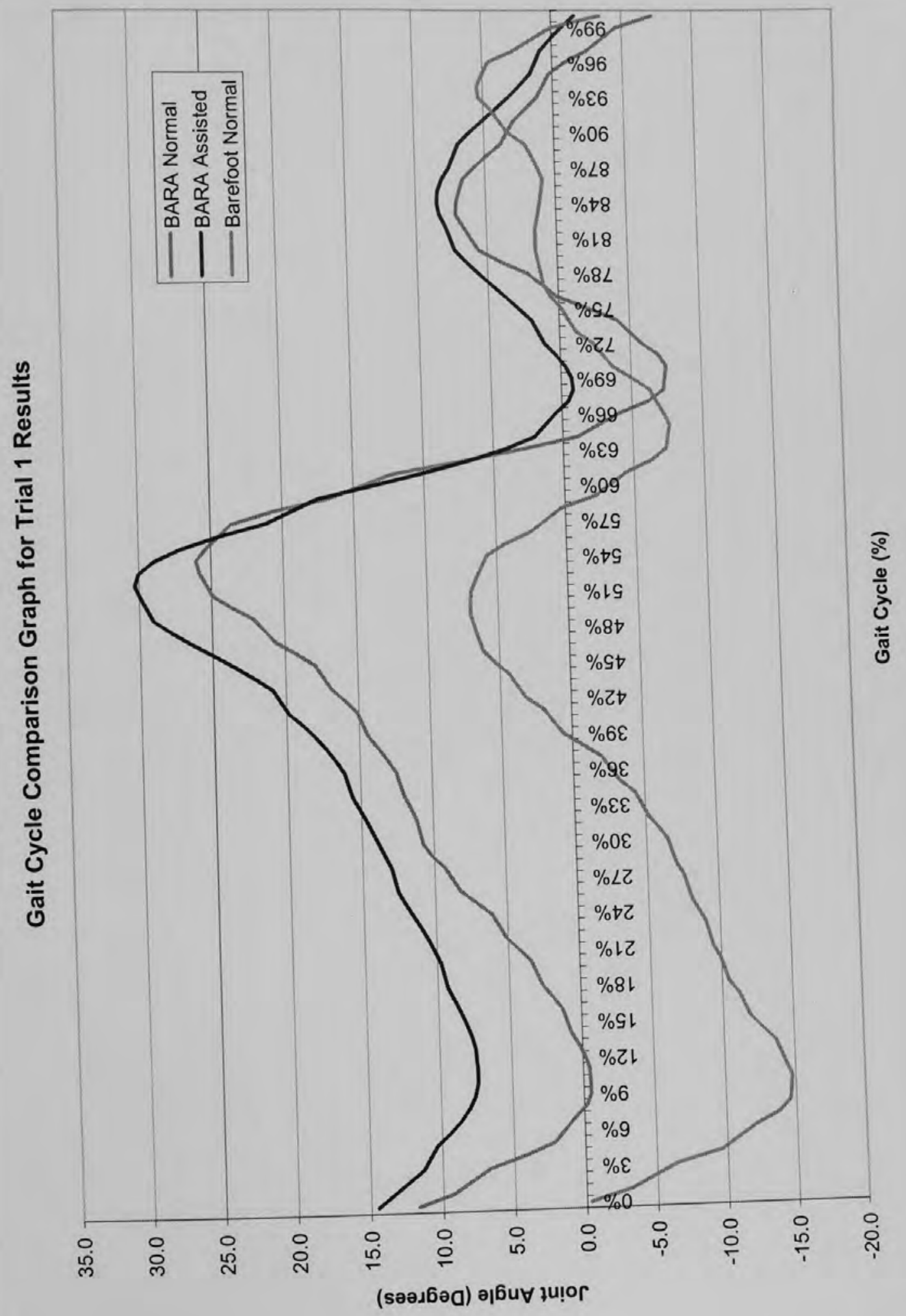

